\title{
A revision of Tripogon (Poaceae: Chloridoideae) in India
}

\author{
Thoiba K.* \& A.K. Pradeep \\ Department of Botany, University of Calicut, Kerala - 673 635, India. \\ *E-mail: thoibakk@gmail.com
}

\begin{abstract}
A taxonomic account of Tripogon Roem. \& Schult. in India is provided, in which 24 species and two varieties are recognised. The taxonomic status of several Indian taxa is updated. Lectotypes are designated for two names ( $T$. longearistatus Hack ex Honda and $T$. zeylanicus Nees ex Steud.). Seven taxa (T. copei Newmaster, V.Balas., Murug. \& Ragup., T. jayachandranii Arum. \& Murugan, Tripogon nallamalayanus Rasingam \& J.Swamy, T. paramjitianus Murug., Arum. \& Kabeer, T. tirumalae Chorghe, Rasingam, Prasanna \& Sankara Rao, T. umae-ganeshii B.R.P.Rao \& M.Anil Kumar, and T. wightii var. kanyakumariensis Kabeer \& V.J.Nair) are reduced to synonymy and T. longearistatus Hack. ex Honda is reported for the first time from India. Descriptions illustrations, distribution, habitat data and identification keys to the species and varieties are provided.
\end{abstract}

Keywords: Lectotypifications, New record, Synonymy, Taxonomy.

\section{Introduction}

Tripogon Roem. \& Schult. (Poaceae, Chloridoideae, Cyanodonteae) is a small genus of grasses with $c$. 51 species occurring in tropical, subtropical and warm temperate regions of Africa, America, Asia and Australia (Phillips \& Launert, 1971; Phillips \& Chen, 2002; Rúgolo \& Vega, 2004; Fabillo, 2015). Twenty four species and two varieties are recorded in India. The genus is characterised by unilateral racemes and laterally compressed spikelets with 3-nerved lemmas (Clayton \& Renvoize, 1986). Species of Tripogon occur in a variety of habitats, most of them in open habitats adjoining humid

Received: 27.10.2019; Revised \& Accepted: 02.07.2020

Published Online: 30.09.2020 shola forests and prefer to grow on cliffs of moist rocks, wet granitic boulders and rocky slopes. A few species (e.g., T. capillatus Jaub. \& Spach) grow as epiphytes on the basal parts of tree trunks in humid evergreen forests, while several others occur in xeric to dry habitats with poor soil. Since Bor's (1960) account on the grasses of the Indian subcontinent, several species were added to the genus in India by various authors (Sreekumar et al., 1983a, 1983b; Pradeep \& Sunil, 1999; Sunil \& Pradeep, 2001; Murugesan \& Balasubramaniam, 2008; Newmaster et al., 2008; Kabeer et al., 2009; Chorghe et al., 2013, 2015; Thoiba \& Pradeep, 2014; Sunil et al., 2015; Thoiba et al., 2015, 2016; Arumugam \& Murugan, 2017; Murugesan et al., 2017; Rao \& Anil Kumar, 2018; Rasingam \& Swamy, 2018) increasing the number of species from 13 to 24, excluding those that have been reduced into synonymy. Peterson et al. (2016) in their recent classification based on molecular phylogeny erected a new segregate genus, Tripogonella P.M.Peterson \& Romasch. from Tripogon and concluded it is clearly polyphyletic with two separate clades "Tripogonella" and "Eragrostiella”. Tripogon species are often superficially extremely similar, and can easily be differentiated from other grass genera by their unique spikelets (Phillips \& Chen, 2002). However, species determination may often pose difficulties but can be done with ease once identifying characters have been properly defined. There has not been any significant work on the taxonomy of Tripogon since N.L. Bor's (1960) monumental work on The Grasses of Burma, Ceylon, India and Pakistan. The present revision provides an updated taxonomic account on the genus Tripogon in India. 


\section{History}

The name Tripogon is derived from the Greek word, 'treis' (three) and 'pogon' (beard) referring to "lemmas have three apical awns and three basal hair-tufts" (Watson et al., 1992; Clifford \& Bostock, 2007). The genus was originally established by Roemer and Schultes (1817) as "Calyx 2-valved, somewhat 8-flowered, somewhat awned. Corolla 2-valved, outer valve with a pilose base, below the apex with a straight awn, with margin at the middle on both sides provided with an outward bending awn. Stamens 3. Stigmas 2, feathery. Seed 1" with a reference to A.W. Roth's Triathera in an unpublished manuscript (Nova Plantarum Species). In the same work, Roemer and Schultes described a single species of Tripogon, T. bromoides with a reference to Triathera bromoides Roth. The authors commented "primo intuitu similis FESTUCAE myuro" that their new species is at first glance similar to Festuca myuros L. It is evident that Roemer and Schultes (1817) have considered Festuca mysorensis as synonymous to $T$. bromoides and has indicated "H. in Mysore". Since, T. bromoides was the only species included while establishing the genus, it constitutes the type species of the genus. However, no reference to a specimen was given in the protologue. The reference to " $\mathrm{H}$. in Mysore" in the protologue indicates Heyne in Mysore. Benjamine Heyne, a German missionary serving as superintendent of Bangalore Garden in 1802 (Stafleu \& Cowan, 1979), probably collected the specimen from Mysore in India. Both Heyne and A.W. Roth's types are at B and it seems Roth received Heyne's specimen for identification and it became part of Roth's Herbarium. Roth identified it as Festuca mysorensis (unpublished) and later Roemer \& Schultes gave it a new name, $T$. bromoides. A well-preserved specimen evidently collected by Heyne in 1814 from Mysore is available at Berlin's Botanic Garden and Botanical Museum herbarium (B100367364 digital image!), with a handwritten (presumably by Roth) note on Festuca mysorensis Heyne affixed on it. Since it is the only element that can be attributed with some certainty to the original collection of Tripogon bromoides, it is accepted as the holotype of T. bromoides Roem. \& Schult.

\section{Growth form and habitat}

The majority of Indian Tripogon species are caespitose, slender or stout, erect, annuals or perennials, a few species (T. vellarianus Pradeep, T. bimucronatus Thoiba \& Sunil, T. malabaricus Thoiba \& Pradeep) form well-developed or tussock forming rootstocks and much longer racemes, whereas species such as T. velliangiriensis Murug. \& V.Balas. and T. wightii Hook.f. are sometimes stoloniferous. Tripogon pungens C.E.C.Fisch. is peculiar in having short, thick root stocks firmly attached to rock crevices. Tripogon generally thrive in a variety of habitats such as high altitude rocky outcrops (inselbergs), grasslands, and forest margins. The majority of species prefer wet rocky hillsides, steep granitic cliffs, road cuts and boulders along grasslands. Species such as T. capillatus and T. lisboae Stapf often occur on tree trunks in open places along margins of wet evergreen and moist deciduous forests. Tripogon jacquemontii Stapf is usually found on boulders in dry habitats and on walls of old forts, while T. polyanthus Naik \& Patunkar prefers to grow on rocks along river side.

\section{Culms}

The culm nodes vary in number from 1-4. Leaves are arranged all along the culm or grouped at the base of the culms.

\section{Leaf sheath}

The sheath is densely hairy throughout (T. filiformis Nees ex Steud.), tough, imbricate and striate (T. pungens). Frequently the basal sheath of Tripogon breaks up into fibrous threads which persist for a very long time.

\section{Ligule}

The ligules may be ciliate or long pilose hairy (T. trifidus Munro ex Stapf, T. narayanae Sreek., V.J. Nair \& N.C.Nair, T. bromoides) or inconspicuous (T. lisboae, T. vellarianus). 


\section{Leaf blade}

The leaf blade features are important taxonomic characters for the delimitation of species in this genus. They may occur in various combinations on the same plant or change from flat to folded or rolled during different stages of their growth. The leaf blades in all species of Tripogon found in India are usually linear ( $T$. capillatus), linear-lanceolate or flat-convolute (T. bromoides, T. idukkianus Sunil \& Pradeep, T. jacquemontii, T. narayanae, T. ravianus Sunil \& Pradeep, T. sivarajanii Sunil, T. zeylanicus Nees ex Steud.), flat (T. bimucronatus, T. malabaricus, T. vellarianus). Leaf apex of $T$. pungens is very unique in having an aculeate apex. The leaves of $T$. filiformis have dense woolly pubescence throughout the plant, while $T$. purpurascens Duthie is easily recognizable by its purple tinged foliage.

\section{Inflorescence}

The inflorescence is invariably a spike-like raceme, but the number and size of spikelets may vary from species to species. Sometimes the racemes may be rigid (T. bimucronatus, T. malabaricus, T. vellarianus), stout (T. lisboae, T. jacquemontii, T. sivarajanii), slender (T. bromides, T. wightii, T. zeylanicus), or flexuous (T. capillatus, T. longearistatus Hack. ex Honda).

\section{Spikelets}

The number of florets and awns is an important feature for the delimitation of species in Tripogon. Disarticulation of florets may be acropetal or basipetal or in between florets. The shape of the glumes in Tripogon varies from species to species and is used as a key character for species delimitation. The lower glume has a lobe or notch on one side, and the upper glumes are represented by bi-lobed teeth with mucronate or miniature awns at the apex (Tripogon bromoides, T. ravianus, T. sivarajanii, T. velliangiriensis, T. wightii, T. zeylanicus). The lemma apex may be 2-lobed (T. bimucronatus, T. lisboae, T. jacquemontii, T.polyanthus, T. malabaricus, T. sivarajanii, T. wightii, T. velliangiriensis) with a single awn, 4-lobed (T. bromoides, T. filiformis, T. trifidus,
T. narayanae, T. munnarensis Thoiba \& Manudev, T. karnatakensis Thoiba \& Pradeep, T. idukkianus) or 6-lobed ( $T$. borii Kabeer, V.J.Nair \& G.V.S.Murthy, T. zeylanicus) with three awns. Similarly, the apex of the palea may vary from species to species. For example, it may be acute (T.polyanthus), bi-lobed (T.capillatus, T. narayanae), bi-mucronate (T. bimucronatus), conduplicate (T. pungens), subulate (T. malabaricus), or with subapical setae (T. idukkianus) (Figs. 1-4).

\section{Caryopsis}

The caryopses are narrow, sub-terete to trigonous in shape. In some species, seed setting is very low

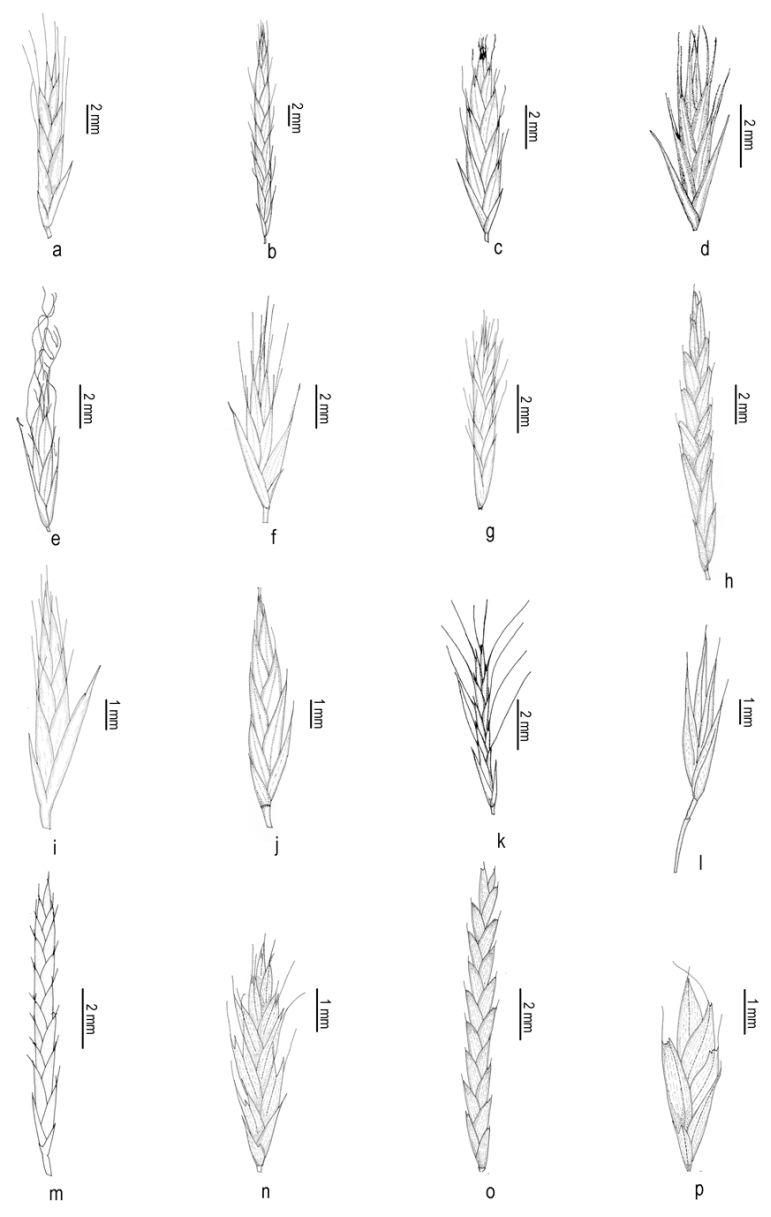

Fig. 1. Spikelets of Tripogon Roem. \& Schult.: a. Tripogon bimucronatus; b. T. borii; c. T. bromoides; d. T. bromoides var. longifolius; e. T. capillatus; f. T. filiformis; g. T. idukkianus; h. T. jacquemontii; i. T. karnatakensis; j. T. lisboae; k. T. Iongearistatus; I. T. malabaricus; m. T. munnarensis; n. T. narayanae; o. T. polyanthus; p. T. pungens. 


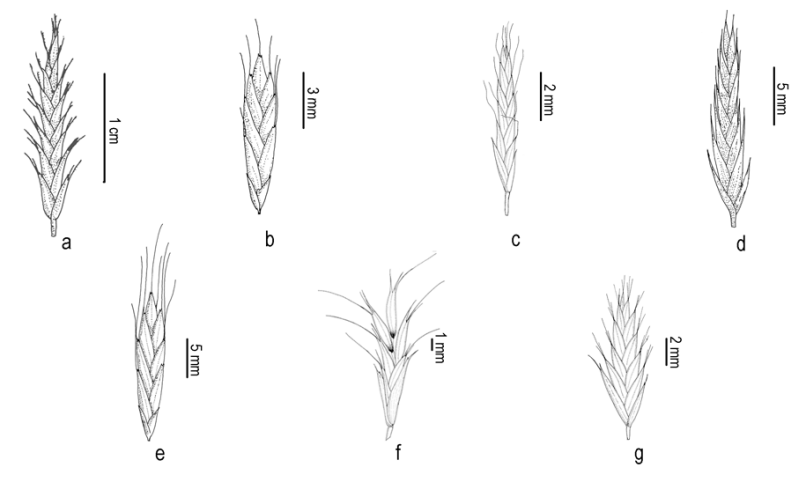

Fig. 2. Spikelets of Tripogon Roem. \& Schult.: a. Tripogon ravianus; b. T. sivarajanii; c. T. trifidus; d. T. vellarianus; e. T. velliangiriensis; f. T. wightii; g. T. zeylanicus.

and poor. Among the 24 species of Tripogon studied from India, only 20 species had mature caryopses. All the caryopses were collected from the field trips in different parts of India (except T. mahendragiriensis Chorghe, Sang.Dey, K.Prasad, Prasanna \& Y.V.Rao). The colour of mature caryopsis may vary from golden yellow to brownish, shining with finely to prominently reticulate surface cells. The length of the caryopses range from $0.2 \mathrm{~mm}(T$. pungens) to $3.5 \mathrm{~mm}$ (T. vellarianus) and their shape varies from narrowly oblong, elliptic-ovate, linearoblong, oblong to elliptic, to oblong cylindrical.

\section{Materials and Methods}

The present treatment is based primarily on the authors' collections and field observations of most taxa, supported by herbarium specimens housed in the following herbaria: AHMA, BLAT, BSI, BSID, CAL, CALI, DEV, FRC, Goa University Herbarium, JCB, KFRI, MH, RHT, SNM College Herbarium, SUK, and TBGT. The acronyms of herbaria are as per Thiers (continuously updated). Habitat and distributional data were collected during field studies and were also recorded from herbarium labels. Morphological studies and measurements were taken with Leica EZ4HD 3.0 digital stereo microscope with Leica application suite version 2.0 and Leica M80 stereo microscope (Mannheim, Germany) attached with camera. Dried materials were examined after softening in warm water.
Synonyms given were based on a critical examination of relevant protologues, types and synonymy lists from the taxonomic literature in India and adjoining regions. The illustrations and plates were prepared by the first author (except for Tripogon idukkianus).

\section{Taxonomic treatment}

Tripogon Roem. \& Schult., Syst. Veg. 2: 34. 1817.

Type: Tripogon bromoides Roem. \& Schult.

Plagiolytrum Nees in Proc. Linn. Soc. 1: 95. 1841, nom. inval.

Annual or perennial. Leaf blades narrow, often rigid (apex pungent in T. pungens); ligules a narrow membrane fringed with hairs or absent. Inflorescence a single unilateral raceme. Spikelets laterally compressed, broadside to the rachis, linear to elliptic, rachilla elongate between the florets, disarticulating above the glumes and tardily so between the florets; callus minute, obtuse, bearded. Glumes membranous, glabrous; lower glumes 1nerved, acuminate, smooth; upper glumes sometimes 3-nerved (the lowest lemma sterile and glume-like in T. siamensis Bor and T. wardii Bor); florets several, closely imbricate, perfect or the upper ones staminate or barren, or rarely the lower floret also barren. Lemmas lightly keeled or rounded, glabrous, bidentate (sometimes entire in T. major Hook.f.), mucronate or awned, the awn straight or rarely flexuous, often the teeth also awned. Paleas hyaline, entire, ciliolate on the keels upwards, otherwise glabrous, sometimes winged. Stamens 13, linear-oblong. Caryopses sub-terete, obtusely triquetrous, narrow, free within the lemma and palea.

Distribution: Widely distributed in tropical and subtropical regions of Africa, Asia, Australia, and the Americas, from southern U.S.A. to Argentina and Sri Lanka. About 51 species in the world; 24 in India of which 22 occur in Peninsular India, one each in Odisha and Meghalaya.

Key to the species of Tripogon in India 

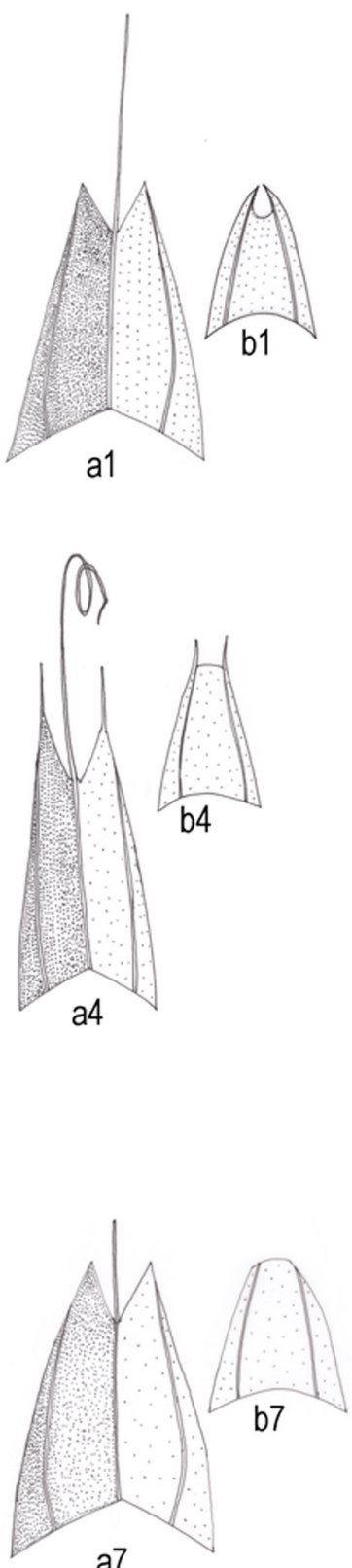

a7

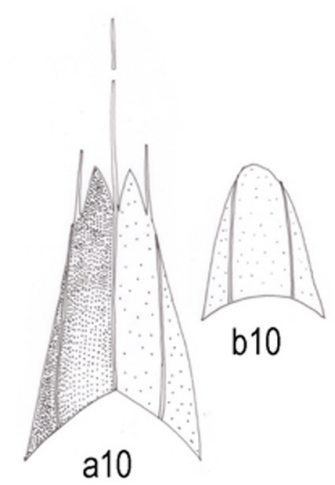

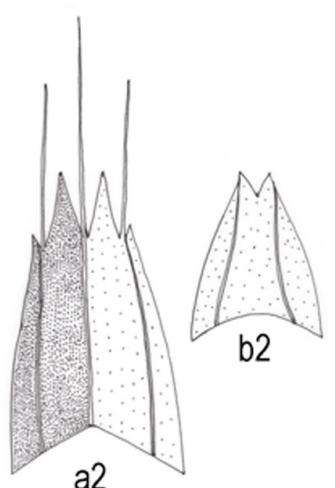

a2
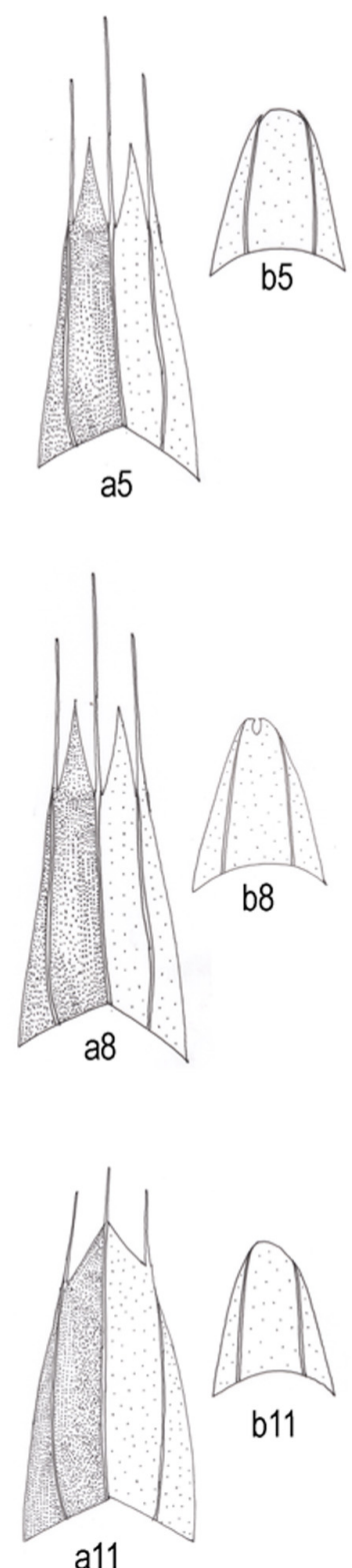
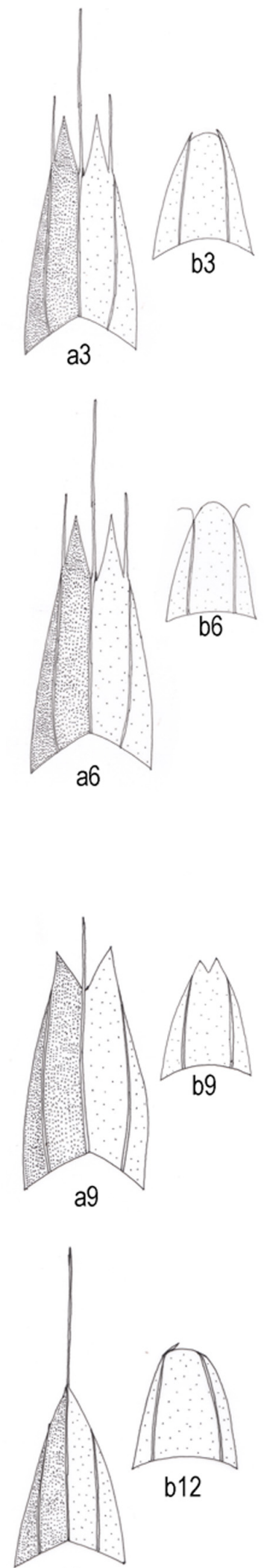

a12

Fig. 3. Diagrammatic representation of the variation in lemma (a) and palea (b) apices in Tripogon Roem. \& Schult.: a1 b1. Tripogon bimucronatus; a2 b2. T. borii; a3 b3. T. bromoides; a4 b4. T. capillatus; a5 b5. T. filiformis; a6 b6. T. idukkianus; a7 b7. T. jacquemontii; a8 b8. T. karnatakensis; a9 b9. T. lisboae; a10 b10. T. Iongearistatus; a11 b11. T. mahendragiriensis; a12 b12. T. malabaricus. 

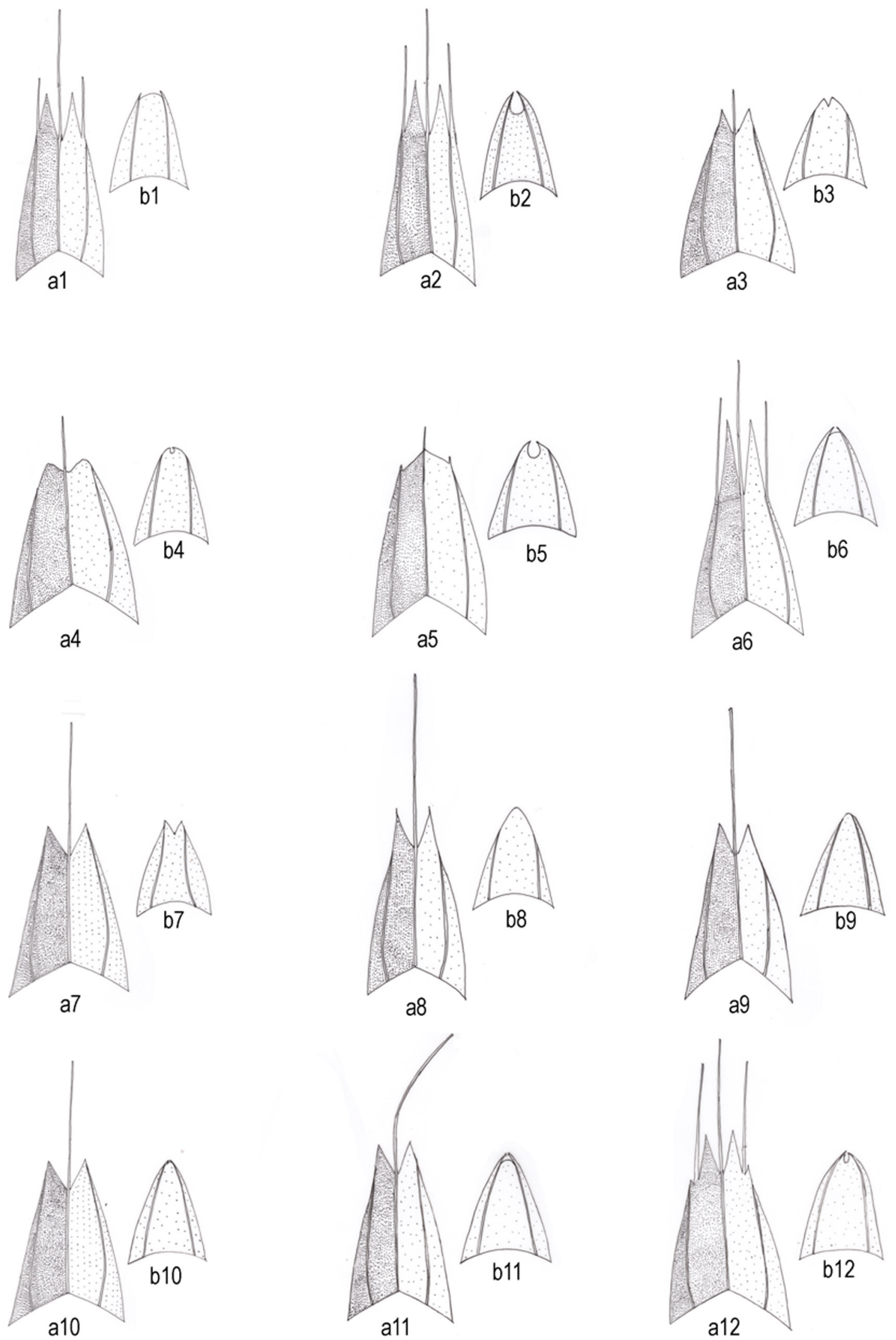

Fig. 4. Diagrammatic representation of the variation in lemma (a) and palea (b) apices in Tripogon Roem. \& Schult.: a1 b1. T. munnarensis; a2 b2. T. narayanae; a3 b3. T. polyanthus; a4 b4. T. pungens; a5 b5. T. purpurascens; a6 b6. T. ravianus; a7 b7. T. sivarajanii; a8 b8. T. trifidus; a9 b9. T. vellarianus; a10 b10. T. velliangiriensis; a11 b11. T. wightil; a12 b12. T. zeylanicus. 
1. Culms thickened below by the persistent leaf sheaths; leaves equitant, rigid and pungent ... T. pungens

1. Culms and leaves not as above 2

2. Lemmas cleft at the apex into 2 lobes, awned in the cleft. 3

2. Lemmas cleft at the apex into 4-6 lobes or not, with or without a definite lobe between each lateral awn and the median awn 14

3. Median awn as long as or longer than the lemmas 4

3. Median awn shorter than the lemma, occasionally almost absent 8

4. Awns straight or curved, not more than twice as long as the lemmas.... 5

4. Awns flexuous, capillary, several times as long as the lemmas T. capillatus

5. Culms stoloniferous; paleas broadly winged .

5. Culms not as above; paleas narrowly winged 7

6. Rachilla 1-1.2 mm long; upper glumes awned, 1-1.5 mm long, lemma awns straight at young stage and recurved at maturity ........ T. wightii

6. Rachilla 0.5-1 mm long; upper glumes mucronate, 0.3-0.4 mm long, lemma awns almost straight but slightly bend at maturity. T. velliangiriensis

7. Ligules a densely ciliate membrane, often glabrous when dry; spikelets up to 20flowered; lemma awns flexuous or recurved, lobes awned or not at the apex ....... T. trifidus

7. Ligules a fine glabrous membrane; spikelets up to 8-flowered; lemma awns stout, lobes never awned at the apex. T. sivarajanii

8. Tufted perennials with wiry-root forming tufts; leaves glabrous or sparsely hairy; plant greenish (except $T$. polyanthus); racemes stout or rigid 9

8. Caespitose perennials with fibrous roots, forming a close turf; leaves covered with a mass of matted hairs; whole plant with a purple tinge; racemes slender T. purpurascens

9. Leaves and culms glaucous; leaves convolute or involute, 4-30 cm long; lower glumes asymmetrical.

9. Leaves and culms not glaucous; leaves flat, 30$70 \mathrm{~cm}$ long; lower glumes symmetrical (except T. bimucronatus, T. lisboae) 11

10. Leaves sub-filiform, involute, longer than or equal to racemes; spikelets 7.5-16 $\mathrm{mm}$ long, 4-25-flowered; common on barren uplands and old walls

T. jacquemontii

10. Leaves linear, convolute, shorter than racemes; spikelets 2-6.5 cm long, 20-60-flowered; more common in river bed rocks .......T. polyanthus

11. Ligules absent or indistinct; glumes acuminate, unawned 12

11. Ligules membranous or ciliate; glumes awned; if unawned, then the plants strongly tussock forming with large spikelets on the racemes.

12. Leaves flat or sometimes rolled; ligules absent; upper glumes shortly aristate; palea acute at apex T. lisboae

12. Leaves flat; ligules indistinct; upper glumes acute; palea subulate at apex .... T. malabaricus

13. Lower glumes asymmetrical; upper glumes mucronate or shortly awned; paleas bimucronate at apex T. bimucronatus

13. Lower glumes symmetrical; upper glumes acuminate; paleas obtuse at apex ... T. vellarianus

14. Lemmas 3-awned with a definite lobe between the awns; anthers 3 15

14. Lemmas 3-awned without definite lobes between the awns; anthers 2 T. mahendragiriensis

15. Lemmas 4-lobed 16

15. Lemmas 6-lobed 23 
16. Median awn shorter than or nearly equal to the lemmas; stout grass

16. Median awn twice as long as the lemma or more; slender grass

17. Lateral awns of lemma half to median awns . 18

17. Lateral awns of lemma shorter than to median awns 19

18. Spikelets $5-10 \mathrm{~mm}$ long, loosely spaced on the rachis, 4-6-flowered; lower glumes 2.3-3.5 $\mathrm{mm}$ long T. narayanae

18. Spikelets 10-16 $\mathrm{mm}$ long, contracted on the rachis, 10-16-flowered; lower glumes 1-2.7 mm long

T. munnarensis

19. Leaves hairy, spikelets compact; paleas emarginate or bi-lobed with a central notch at apex

T. bromoides

19. Leaves sparsely hispid; spikelets distant; paleas acute with sub-apical setae at apex

T. idukkianus

20. Culms and awns stout; leaf blades flatconvolute

20. Culms and awns flexuous or slender; leafblades filiform or convolute 22

21. Rachilla persistent; lower glumes 2-nerved, 2keeled; lemma lobes acute to acuminate

T. karnatakensis

21. Rachilla not persistent; lower glumes 1-nerved, 1-keeled; lemma lobes lanceolate to acuminate T. ravianus

22. Leaf blades densely pilose on both surfaces; racemes with closely imbricate spikelets; lateral awns arising from lemma tooth; anthers 3 ....

T. filiformis

22. Leaf blades glabrous or loosely pilose adaxially and glabrous abaxially; racemes with widely spaced spikelets; lateral awns smaller than the median awn, arising free from lemma tooth or from its outer margins; anther 1

T. longearistatus
23. Spikelets 3-3.5 mm wide; lower glumes 4-5 $\mathrm{mm}$ long; upper glumes 7-9 mm long; anthers 1.3-2 $\mathrm{mm}$ long T. zeylanicus

23. Spikelets 1-2 mm wide; lower glumes 1.8-2.3 $\mathrm{mm}$ long; upper glumes 2.3-4 $\mathrm{mm}$ long; anthers $<1 \mathrm{~mm}$ long T. borii

Tripogon bimucronatus Thoiba \& Sunil, Gard. Bull. Singapore 67(1): 151. 2015. Type: INDIA, Kerala, Palakkad district, Nelliyampathy hill top, 1200 m, 16.11.2010, C.N. Sunil 4477 (holo SING!; iso BRIT!, CAL!, CALI [CALI112088!], K).

Figs. 5 \& 8

Tufted perennials. Culms 30-60 cm high, erect; nodes glabrous. Leaf sheaths closely involute to the culm, rigid, sparsely hairy; ligules ciliate, 2-2.5 mm

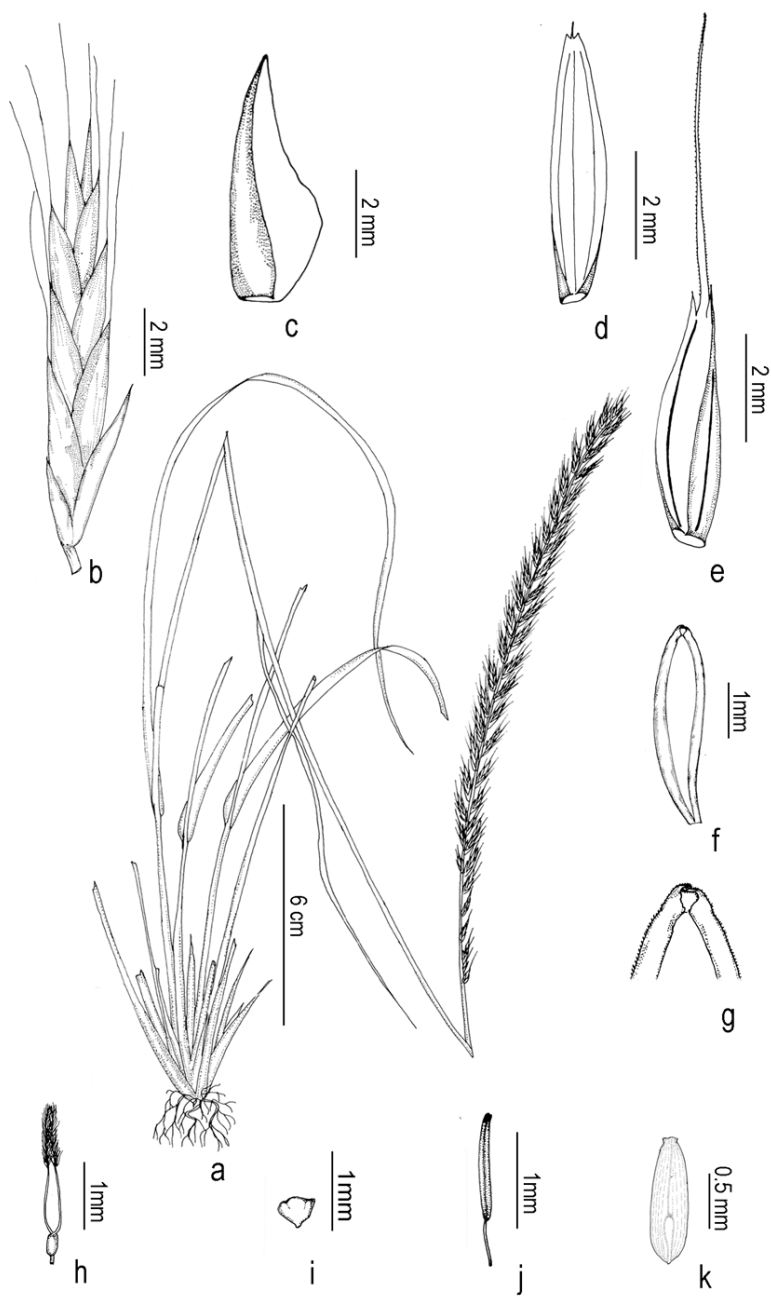

Fig. 5. Tripogon bimucronatus Thoiba \& Sunil: a. Habit; b. Spikelet; c. Lower glume; d. Upper glume; e. Lemma; f. Palea; g. Enlarged portion of palea apex; h. Pistil; i. Lodicule; j. Stamen; k. Caryopsis. 
long; leaf blades 30-60 × 0.4-0.6 cm, linear, hispid towards base, scabrid adaxially, margins involute, apex acute-acuminate. Racemes 30-40 cm long, rigid, straight or slightly curved, 45-65 spikelets tightly appressed to concavities in rachis; rachis stout, minutely scaberulous, triangular; peduncles 10-15 cm long, glabrous, terete. Spikelets 1.3-1.8 $\times 0.2-0.25 \mathrm{~cm}$, linear-lanceolate, 6-8-flowered; callus bearded; rachilla not persistent, hidden, 1-2 mm long, slightly scabrid; lower glumes 3-3.5 × c $1 \mathrm{~mm}$, lanceolate, asymmetrical, notched on oneside below the middle, chartaceous, 1-nerved, acute-acuminate at apex; upper glumes $5-6 \times c .1$ $\mathrm{mm}$, elliptic-lanceolate, prominently 3 -nerved, glabrous, apex mucronate or shortly awned, awns 0.5-1 mm long; lemmas 5-5.5 × 1.5-2 mm (excluding awn), 3-nerved, 1-awned, awn arising between lateral lobes, median awn 5-5.5 mm long, scabrid, straight or geniculate, lateral awns absent; paleas 4-5 × 1-2 mm, hyaline, narrowly elliptic, keeled, winged, keels minutely puberulous, apex bi-mucronate, notched at centre; lodicules 2, c. 0.25 mm long, quadrate, apex coarsely 3-toothed; stamens 3, anthers 1-1.5 mm long, oblong, filaments $0.5-0.75 \mathrm{~mm}$ long, slender, glabrous; ovary $0.25-0.5 \mathrm{~mm}$ long, obovate, styles $2,1 \mathrm{~mm}$ long, slender, hyaline, stigma 1-1.5 mm long. Caryopses $1.1-1.3 \times 0.4-0.5 \mathrm{~mm}$, narrowly oblonglanceolate, obtusely trigonous, longitudinally ribbed abaxially, pale brown, obtuse at apex.

Flowering $\&$ fruiting: Flowering and fruiting from September to November.

Habitat: It usually grows on granitic grassy slopes at elevations between 1000-1200 m, in association with Cyanotis papilionacea (Burm.f.) Schult. \& Schult.f. (Commelinaceae), Arthraxon castratus Bor, Chrysopogon nodulibarbis (Hochst.) Henrard, Cymbopogon commutatus Stapf, Eulalia trispicata (Schult.) Henrard, Garnotia elata Janowsky, Isachne bourneorum C.E.C.Fisch. and Tripogon wightii (all Poaceae), Osbeckia spp. (Melastomataceae), Sopubia trifida D.Don (Scrophulariaceae), Swertia angustifolia D.Don. (Gentianaceae) and Tephrosia pulchella Hook.f. (Fabaceae).

\section{Distribution: Endemic to South India.}

Specimens examined: INDIA, Kerala, Idukki district, Meenuliyanpara, Vannappuram, 28.11.2014, C.N. Sunil 4910 (SNM College Herbarium, Kerala); Ibid., 20.12.2016, F. Dani s.n. (CALI).

Notes: Tripogon bimucronatus is similar to $T$. vellarianus but differs by its asymmetrical lower glumes, mucronate or shortly awned upper glumes and bi-mucronate paleas.

Tripogon borii Kabeer, V.J.Nair \& G.V.S.Murthy, Bull. Bot. Surv. India 50(1-4): 115. 2009. Type: INDIA, Tamil Nadu, Kanyakumari district, Nagerkoil, Maruthwamala, \pm 360 m, 30.11.2005, K. Althaf Ahamed Kabeer 118632 (holo CAL; iso $\mathrm{MH}[\mathrm{MH} 00003227 !])$.

Figs. 6-8

Caespitose perennials. Culms up to $60 \mathrm{~cm}$ high, terete; nodes glabrous, slightly geniculate. Leaf sheaths $5-10 \mathrm{~cm}$ long, linear, glabrous or hairy, apex densely ciliate; ligules membranous, with a tuft of 1-2.5 mm long hairs at apex; leaf blades $25-47 \times$ $0.2-0.4 \mathrm{~mm}$, linear, flat-involute, nerves prominent, adaxially glabrous to sparsely long hairy, abaxially finely scabrellate along ribs, attenuate at apex. Racemes 15-28 cm long, stiff, straight to curved, spikelets appressed to concavities in rachis; rachis stout, glabrous, ribbed; peduncles 5-14 cm long, terete, glabrous. Spikelets 10-16 × 1-2 mm, ovatelanceolate, linear, creamy, 6-14-flowered; callus bearded, hairs $0.5-1 \mathrm{~mm}$ long; rachilla up to 0.7 $\mathrm{mm}$ long, oblique at apex; lower glumes 1.8-2.3 $\times$ 0.5-0.8 mm, oblong, deeply lobed below middle along one side, lobes acute to acuminate, 1-nerved,

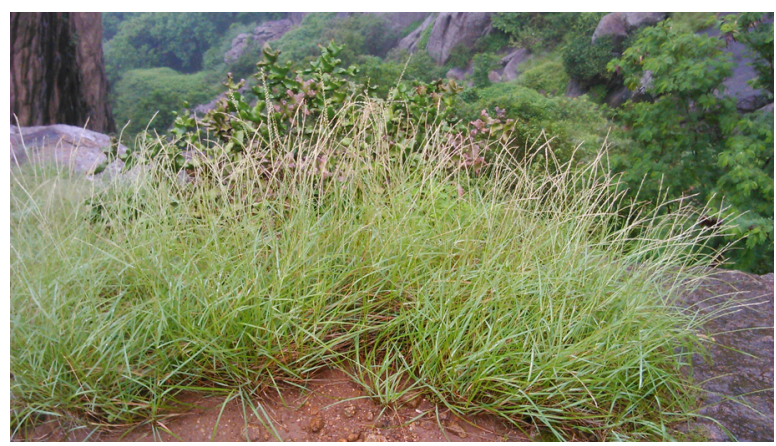

Fig. 6. T. borii Kabeer, V.J.Nair \& G.V.S.Murthy in its natural habitat at Maruthwamala in Kanyakumari district. (Photo by K. Thoiba). 


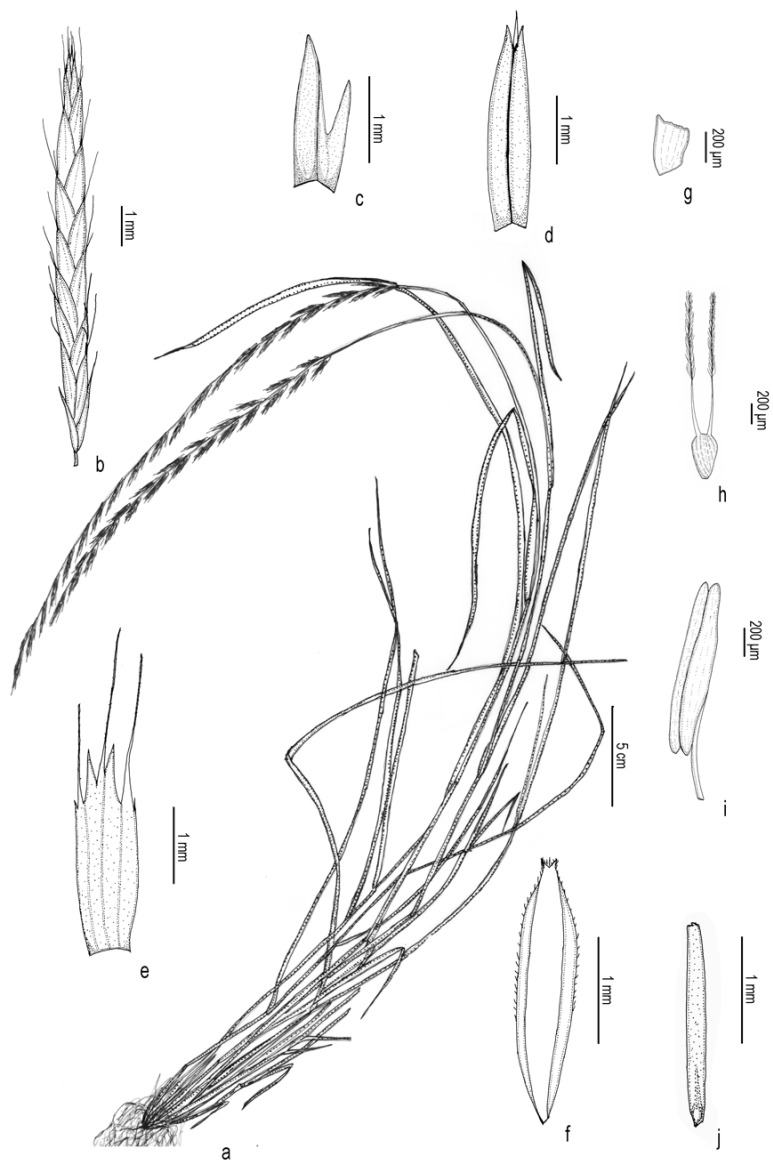

Fig. 7. Tripogon borii Kabeer, V.J.Nair \& G.V.S.Murthy: a. Habit; b. Spikelet; c. Lower glume; d. Upper glume; e. Lemma; f. Palea; g. Lodicule; h. Pistil; i. Stamen; j. Caryopsis.

1-keeled, keels slightly scabrid, acute and mucronulate at apex; upper glumes $2.3-4 \times c .0 .8 \mathrm{~mm}$, elliptic-oblong, 1-keeled, 1-nerved, apex bi-lobed, aristate, arista $0.5-2 \mathrm{~mm}$ long at sinus; lemmas 2.8-4 × 0.8-1 mm (excluding awn), oblonglanceolate, 6-lobed, 3-nerved, 3-awned, median awns 2.5-3.5 mm long; lobes on either side of median awns acute to acuminate, 0.4-1 mm long, lateral awns 1.5-2.5 mm long, lateral lobes on either side conspicuous, slightly keeled; paleas $2.3-2.8 \times$ 0.8-1 mm, oblong-elliptic, hyaline, 2-nerved, 2-keeled, keels ciliate, sub-acute at apex; lodicules 2, 0.3-0.5 mm long, truncate; stamens 3 , anthers 0.7-1 mm long, oblong, purple tinged at fresh, filaments $0.5-0.75 \mathrm{~mm}$ long, slender, glabrous; ovary $0.3-0.4 \mathrm{~mm}$, oblong-elliptic, styles $2,1-1.2$ mm long, slender, hyaline, stigma 0.7-1 mm long, plumose, creamy white. Caryopses $1.5-1.8 \times 0.2-$ $0.3 \mathrm{~mm}$, narrowly oblong-lanceolate, light brown.

Flowering \& fruiting: Flowering and fruiting from September to early December.

Habitat: This species prefers to grow in rock crevices and on seasonally wet bare rocks in high elevation grasslands in association with Anisochilus carnosus (L.f.) Wall., Leucas nepetifolia Benth. (both Lamiaceae), Cyanotis cristata (L.) D.Don (Commelinaceae), Arundinella pumila Steud., Cymbopogon citratus Stapf, Eragrostiella bifaria (Vahl) Bor, Tripogon bromoides (all Poaceae), Henckelia incana (Vahl) Spreng. (Gesneriaceae) and Justicia sp. (Acanthaceae).

Distribution: Endemic to South India.

Specimens examined: INDIA, Tamil Nadu, Coimbatore district, Velliangiri hills, 30.09.2016, A.K. Pradeep, Nikhil Krishna \& K. Thoiba 146727; Ibid., 30.09.2016, A.K. Pradeep, Nikhil Krishna \& K. Thoiba 146728, 146729 (CALI); Kanyakumari district, Maruthwamala, 09.08.2014, K. Thoiba 138094; Ibid., 30.10.2015, K. Thoiba\& T. Mohammed Yoonus 146650, 146658, 146660, 146662, 146665 (CALI); Nagercoil, Mambazhathuraiyar reservoir, 29.10.2015, K. Thoiba \& T. Mohammed Yoonus 146648 (CALI).

Notes: Very similar to Tripogon zeylanicus and $T$. bromoides, but differs in having ovate-lanceolate, creamy yellow spikelets with up to 14 florets and lower glumes being deeply lobed below the middle along one-side; upper glumes bi-lobed at apex and 0.5-2 $\mathrm{mm}$ long arista at the sinus.

Tripogon bromoides Roem. \& Schult., Syst. Veg. 2: 600. 1817; Hook.f., Fl. Brit. India 7(22): 287. 1896; C.E.C.Fisch. in Gamble, Fl. Madras 3: 1834. 1934; Bor, Grass. Burma Ceylon India \& Pakistan 521. 1960; Britto \& K.M.Matthew in K.M.Matthew, Fl. Tamilnadu Carnatic 3(2): 1911. 1983; Karth., S.K.Jain, M.P.Nayar \& Sanjappa, Fl. Ind. Enum. Monocot. 622. 1989; Sreek. \& V.J.Nair, Fl. Kerala Grass: 399. 1991; M.D.Dassanayake, F.R.Fosberg \& W.D.Clayton, Revis. Handb. Fl. Ceylon 8: 443. 1994; S.Moulik, Grass. Bam. India 2: 622. 1997; 


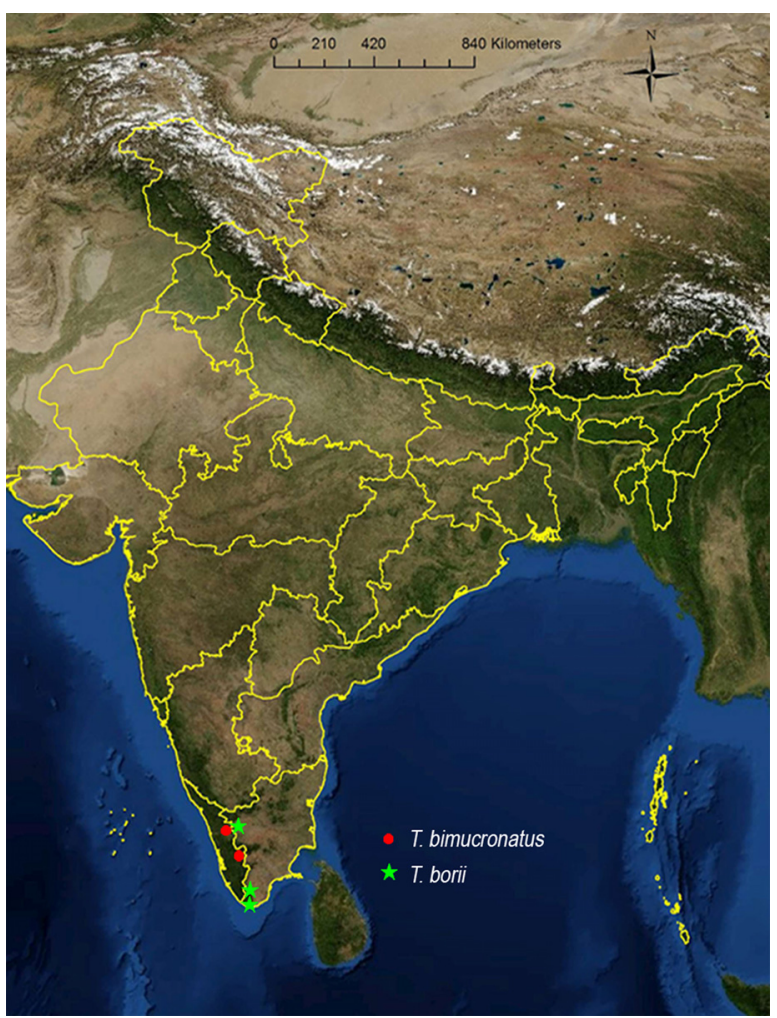

Fig. 8. Distribution of Tripogon bimucronatus Thoiba \& Sunil and $T$. borii Kabeer, V.J.Nair \& G.V.S.Murthy in India [drawn using ESRI (2011) ArcGIS Desktop: Release 10].

Pull., Fl. Andhra Pradesh 3: 1268. 1997; Sasidh., Biodiv. Doc. Kerala-Fl. Pl.: 595. 2004; Kabeer \& V.J.Nair, Fl. Tamil Nadu Grass. 179. 2009; Potdar, Salunkhe \& S.R.Yadav, Grass. Maharashtra: 557. 2012. Avena mysorensis Spreng. Syst. Veg. 1: 337. 1825. Type: INDIA, Karnataka, Mysore, 1814, B. Heyne s.n. (B [B10367364 digital image!]).

Notes: Bor (1960) considered T. bromoides as an "extremely variable grass" and commented on the varieties recognised by Hooker (1896) as "hardly worth keeping". However, the present investigation based on a large number of specimens from its entire range of distribution revealed that one of his varieties (var. longifolius Hook.f.) is worth keeping.

\section{Key to the varieties}

1. Culms 30-70 cm high; leaves villous on both sides; racemes 10-30 cm long; upper glumes shortly aristate or shortly awned, awns $0.3-0.8$ $\mathrm{mm}$ long T. bromoides var. bromoides
1. Culms 5-30 cm high; leaves sparsely villous adaxially and glabrous abaxially; racemes 5$10 \mathrm{~cm}$ long; upper glumes prominently awned, awns 1-2 mm long

T. bromoides var. longifolius

\section{T. bromoides Roem. \& Schult. var. bromoides}

Figs. $9,10 \& 13$

Caespitose perennials. Culms 30-70 cm high, erect; nodes glabrous, slightly geniculate and 1-noded. Leaf sheaths $04-10 \mathrm{~cm}$ long, linear, glabrous or hairy; ligules indistinct with a tuft of 1-2 mm long hairs at apex; leaf blades $15-50 \times 0.3-0.5 \mathrm{~mm}$, linear-lanceolate, flat-convolute, more hairy towards collar, villous along both surfaces, acuminate to attenuate at apex. Racemes $10-30 \mathrm{~cm}$ long, stiff, slender, spikelets tightly or loosely arranged in rachis; rachis 3-7 $\mathrm{mm}$ long, stout, glabrous or scabrid; peduncles $10-25 \mathrm{~cm}$ long, glabrous. Spikelets 7-10 × 2-2.8 mm, linear, compact, dorsiventrally flattened, olive green or yellowish with purple tinge, 5-16-flowered; callus bearded, hairs 1-1.2 mm long; rachilla 1-1.2 mm long, glabrous to scabrid, almost straight, not persistent; lower glumes 1.6-3.5 × 0.75-1.2 mm, ovate-lanceolate, notched on one side, 1-keeled, 1-nerved, keels slightly scabrid, acuminate at apex; upper glumes $2.4-5.8 \times 0.5-1 \mathrm{~mm}$, ellipticlanceolate, 1-keeled, 1-nerved, apex dentate, shortly aristate or awned, awns $0.3-0.8 \mathrm{~mm}$ long; lemmas $2-4.4 \times 1-2 \mathrm{~mm}$ (excluding awns), ovatelanceolate, 4-lobed, lateral lobes and lateral awns

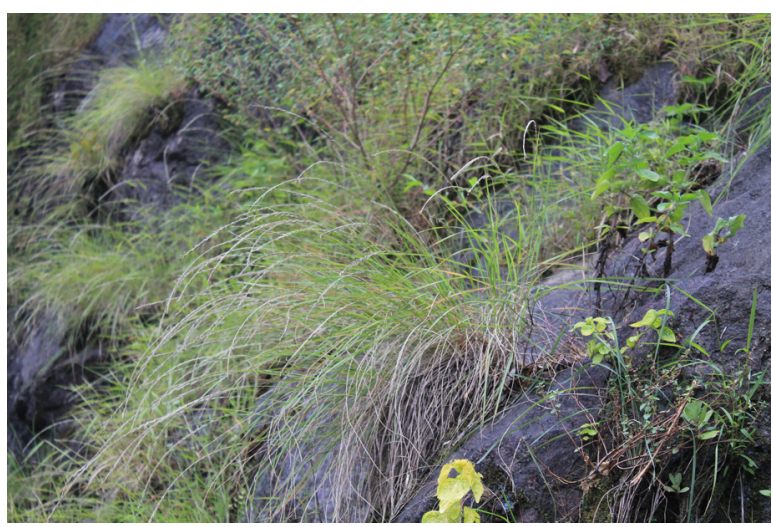

Fig. 9. Tripogon bromoides Roem. \& Schult. var. bromoides in its natural habitat (Photo by K. Thoiba). 
almost equal or sometimes reduced, 3-nerved, slightly keeled, 3-awned, awns scabrid, apex straight or geniculate, median awns 1.5-3.6 mm long, lateral awns $0.5-2.3 \mathrm{~mm}$ long; lateral lobes $0.3-1 \mathrm{~mm}$ long, margins scabrid; paleas $2-4 \times 0.5-$ $1.2 \mathrm{~mm}$, elliptic-oblanceolate, hyaline, 2-keeled, ciliate above the half, purple dotted emarginate or bi-lobed with a minute central notch at apex; lodicules 2, 0.3-0.4 mm long, truncate; stamens 3, anthers 1-1.2 mm long, oblong, filaments 0.4-0.75 $\mathrm{mm}$ long, slender, glabrous; ovary $0.3-0.5 \mathrm{~mm}$ long, obovate, styles 2, 0.5-0.75 mm long, slender, hyaline, stigma $0.75-1.2 \mathrm{~mm}$ long, plumose, creamy white. Caryopses 1-1.6 × 0.3-0.4 mm, narrowly oblong-lanceolate, dark brown.

Flowering \& fruiting: Flowering and fruiting from late July to December.

Habitat: Grows in grasslands, on moist slopes and in rock crevices, along forest margins and Ghat road sides at medium to high elevations, in association with Anisochilus carnosus, Leucas biflora R.Br., Pogostemon mollis Benth. (all Lamiaceae), Arundinella purpurea Steud., Chrysopogon nodulibarbis, Eragrostiella bifaria, Tripogon zeylanicus, T. ravianus, T. sivarajanii (all Poaceae), Henckelia humboldtiana (Gardner) A.Weber \& B.L.Burtt (Gesneriaceae), Exacum tetragonum Roxb. (Gentianaceae), Impatiens acaulis Arn. (Balsaminaceae), Justicia procumbens L. (Acanthaceae), Murdannia semiteres (Dalzell) Santapau (Commelinaceae) and Sonerila gamblei G.S.Giri \& M.P.Nayar (Melastomataceae).

Distribution: India and Sri Lanka.

Specimens examined: INDIA, Andhra Pradesh, Anantapur district, Batrepalli, 14.02.2009, B. Raviprasad Rao \& P. Priyadarsini 37069 (BSID); Nellore district, Rapur Ghat, 09.10.2016, K. Thoiba 146783 (CALI). Jammu \& Kashmir, Kathua district, Bhullari, 20.09.1963, R.S. Rao 91938 (BSI). Karnataka, Bangalore district, 10.06.1953, Govinda CoA 200 (CAL); Bommanahalli, way to Tumkur, 29.09.2015, K. Thoiba 144166; Ibid., 29.09.2015, K. Thoiba 144177 (CALI); Chamarajanagar district,

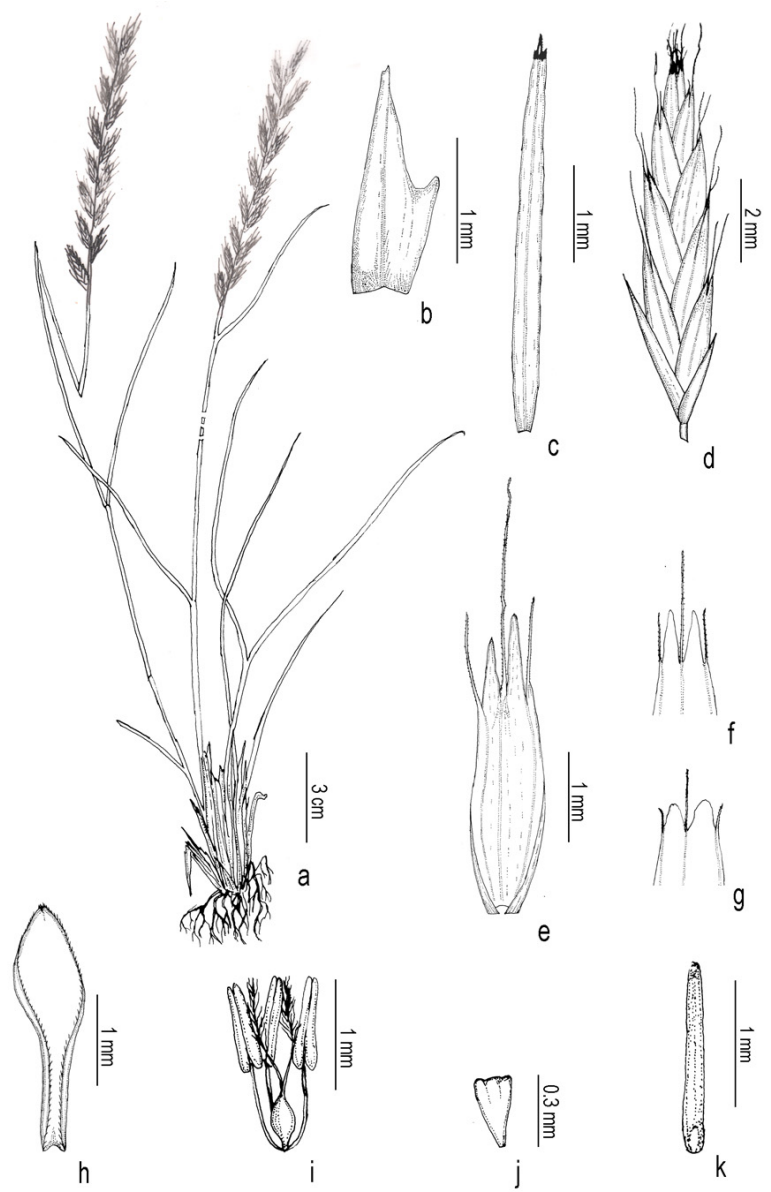

Fig. 10. Tripogon bromoides Roem. \& Schult. var. bromoides: a. Habit; b. Lower glume; c. Upper glume; d. Spikelet; e. Lemma; f. \& g. Lemma apices; h. Palea i. Stamen \& Pistil; j. Lodicule; k. Caryopsis.

Himavad Gopalaswamy hills, 30.09.2015, K. Thoiba 144169 (CALI); Chikmagalur district, way to Baba Budan hills, 13.09.2014, K. Thoiba 137531a; Ibid., 13.09.2014, K. Thoiba 137531b, 137531c (CALI); Kodagu district, Tadiyandamol hills, 26.11.2014, K. Thoiba 137555; Ibid., 26.11.2014, K. Thoiba 137558 (CALI); Mysore district, 06.09.1893, Talbot 3079 (CAL); Tumkur district, Devarayanadurga, 29.08.1961, A.R.K. Shastry 73420 (BSI). Kerala, Ernakulam district, Shoolamudi, Variyam, 14.12.2016, Nikhil Krishna \& K. Thoiba 146740 (CALI); Idukki district, Lockhart gap, 12.10.1963, K.M. Sebastine 17527, 17530 (CAL); Vagamon, way to Pullikanam, 15.10.2015, K. Thoiba \& A.K. Pradeep 146611; Parunthpara, 15.10.2015, K. Thoiba \& A.K. Pradeep 146618; Ibid., 15.10.2015, K. Thoiba \& A.K. Pradeep 146620; Kumaly-Theni road, 16.10.2015, 
K. Thoiba \& A.K. Pradeep 146625; Thrisangu hills, Peerumedu, 17.10.2015, K. Thoiba \& A.K. Pradeep 146637; Ibid., 17.10.2015, K. Thoiba \& A.K. Pradeep 146638, K. Thoiba \& A.K. Pradeep 146643; Munnar, Mattupetty, 16.12.2014, A.K. Pradeep \& K. Thoiba 144104; Cheruthoni, Pinav, ThodupuzhaKattapana road, 17.10.2015, K. Thoiba \& A.K. Pradeep 146633; Munnar, Pallivasal, 14.11.2014, K. Thoiba \& A.K. Pradeep 137565; Devikulam, 16.12.2014, K. Thoiba \& A.K. Pradeep 137588, 137589, 137590, 137591, 137593, 137595 (CALI); Eravikulam National Park, 23.08.1980, P.V. Sreekumar 67772; Ibid., 24.08.1980, P.V. Sreekumar 67780 (BSID, CALI); Peerumedu, 26.09.1964, K. Vivekananathan 21413 (MH); Poovanpara, 07.09.1997, S.D. Biju 36186 (TBGT); Mathikettan Shola National Park, 10.01.2016, K. Thoiba 146719; Ramakkalmedu, 23.10.2017, A.K. Pradeep \& K. Thoiba 146749 (CALI); Kollam district, Ponnambalamedu, 04.07.1981, P.V. Sreekumar 69495 (BSID); Kottayam district, Anaimudi slopes, 19.11.1965, B.V. Shetty 26544 (MH); Kozhikode district, Kakkadampoyil, 21.11.2009, A.K. Pradeep 90074; Ibid., 19.10.2016, Drisya V. \& K. Thoiba 146760; Kozhipara waterfall, 21.11.2009, A.K. Pradeep 90074; Ibid., 09.10.2013, K. Thoiba 134471, 134472; Ibid., 13.10.2017, Nikhil Krishna \& K. Thoiba 146754; (CALI); Palakkad district, Sispara, 28.03.1983, N.C. Nair 77216 (CAL, MH); Silent Valley Dam, near hanging bridge, 05.10.1979, N.C. Nair 64270; Dhoni R.F., 23.10.1963, Joseph 17860 (CAL); Malampuzha dam site, 11.12.2014, K. Thoiba 144129; Nelliyampathy, Seetharkundu, 17.09.2013, K. Thoiba 134435; Ibid., 19.09.2013, K. Thoiba 134439; Kaeshavanpara, 07.11.2013, K. Thoiba 134493; Ibid., 07.11.2015, K. Thoiba 146680; way to Silent Valley, near gateway, 18.09.1982, Sathish Kumar 10754; Silent Valley National Park 25.09.2013, K.M. Manudev \& K. Thoiba 135106; Walaghat, 21.04.1983, Sathish Kumar 11249 (CALI); Sirendhri, Nilgiri Biosphere Reserve, 03.10.2008, J. Remya \& Prasanna 63175; Aruvampara, s.d., Remya J. \& Prasanna 74584 (TBGT); Thiruvananthapuram district, Agasthyamala, Pongalapara, 06.10.1973, J. Joseph
44631; Ibid., 14.03.1988, N. Mohanan 9750; Ibid., 15.05.1988, N. Mohanan 9835 (MH); Ibid., 14.10.1988, N. Mohanan 4279, 4300 (CALI); Athirumala, 04.02.1988, N. Mohanan 8922 (MH); Thrissur district, Parambikulam Tiger Reserve, view point, 24.09.2013, K. Thoiba 134465; Earth dam, 26.09.2013, K. Thoiba 134462; Kariyamchola, 27.09.2013, K. Thoiba 134449; Vengoli hills, 25.09.2013, K. Thoiba 134453, 134460; Malakkapara, Shaekalmudi, Mudiyankunnu, 12.12.2013, K. Thoiba 138026 (CALI); Wayanad district, Myladipara, 22.08.2013, K. Thoiba 134430; Meenangadi, 15.09.2016, Janeesha A.P. \& K. Thoiba 146769; Kurichyarmala, 24.10.2000, Ratheesh Narayanan 2278 (CALI); Pakshipathalam, 06.10.2010, Remya J. \& Prasanna 65492; Ibid., 09.10.2012, Remya J. \& Prasanna 73837; Manikunnumala, 20.08.2011, J. Remya \& Prasanna 67129 (TBGT). Madhya Pradesh, Jabalpur district, 25.11.1989, J.F. Duthie 10038 (CAL). Maharashtra, Aurangabad district, Ajanta caves, 30.09.1963, R.S. Rao 92658 (BSI); Kolhapur district, Radhanagari, 18.10.2007, K.V.C. Gosavi 2805 (SUK); Zenda, 26.10.2004, Aparna Watve A0476 (AHMA); Pune district, Chirka farm, 30.09.1902, s.coll. s.n.; Chatarshingi hill, 02.11.1904, G.A. Gammie s.n.; Katraj Ghat, 30.09.1903, G.A. Gammie s.n.; Ibid., 06.10.1957, G.S. Puri \& Bheodi 25604 (BSI); Khandala, 07.10.1956, R. Fernandez 3001; Purandhar Vazirgarh fort, 08.10.1950, H. Santapau 11362 (BLAT); Ramling, 04.01.1958, S.K. Jain 30922; Shivneri fort, 10.10.1962, R.S. Rao 83517; Sinhagad, 1956, s.coll. 7819; Ibid., 21.10.1964, M.Y. Ansari 101702, 101676 (BSI); Raigad district, Junnar, on the way to Kukadi river, 14.10.1962, M.Y. Ansari 81945; Matheran-Neral road, Samson tank, 17.08.1963, B.M. Wadhwa 67386(BSI); Sindhudurg district, Amboli, Savantwadi, 30.09.1978, S.M. Almedia SMA 1953 (BLAT); Amboli Ghat, 27.11.2006, K.V.C. Gosavi 274; Bhuibavada Ghat, 07.10.2007, K.V.C. Gosavi 2791(SUK). Odisha, Koraput district, Pottangi, 25.08.2013, Alok Chorghe 10235 (BSID). Rajasthan, Ajmer district, Julelia mala block, Mount Alice, 24.10.1960, B.C. Nanda 2091 (CAL); 
Chittorgarh district, Sunna, 21.08.1959, S.K. Jain 58709 (BSI); Pali district, Bheru Ghat, 15.09.1957, G.S. Puri 26566 (BSI). Tamil Nadu, Coimbatore district, Anamalai Tiger Reserve, Nallamudi, Poonjolai, 13.12.2013, K. Thoiba 138016; Velliangiri hills, 13.01.2016, A.K. Pradeep 146718 (CALI); Dindigul district, Berijam slopes, 09.08.1984, K.M. Matthew 40784; Combal's cave, 15.06.1986, Fieldman 49768; Gundur-Vembadi slopes, 27.07.1982, K.M. Matthew \& M. Charles 45903; Ibid., 04.07.1987, K.M. Matthew 49837; Gundur Pumbarai road, 04.11.1987, K.M. Matthew \& K.T. Matthew 51083; Kodai-Berijam road, before fire tower, 07.09.1991, K.T. Matthew 54549 (RHT); Kodai to Kukkal, 07.07.1920, s.coll. 16522 (MH); Kodaikanal, foot of fire tower, 15.06.1990, K.T. Matthew 54304 (RHT); Kodaikanal, Palani hills, 02.07.1898, Bourne 1973; Ibid., 04.07.1901, Bourne 2100, 2111 (CAL); Konalar, 05.11.1987, K.M. Matthew \& K.T. Matthew 51121; Kukkal, 24.08.1986, K.M. Matthew, M. Charles \& N. Rajendren 46597; Palani Ghat road, Kombaikadu, Melpallam, s.d., K.M. Matthew \& N. Rajendren 50325; Palani hills, Perumal peak, 10.06.1986, K.M. Matthew 45618; Periakulam path, joining bridle path, 15.06.1990, K.M. Matthew \& K.T. Matthew 54358; Shembaganur, Periakulam path, 15.05.1982, K.M. Matthew 18067; Shembaganur, Kodai levinge path, 09.07.1986, K.M. Matthew \& N. Rajendren 45772 (RHT); Silver cascade, 26.05.1898, Bourne 3140; Tandigudi-lower Pulneys, 24.05.1899, Bourne 3150 (CAL); upper Pulney, 15.09.1911, Fisher 2998 (FRC); Vandaravu-Marion shola levinge path, 18.12.1989, K.M. Matthew \& K.T. Matthew 54054; Vadakavunchi, Kodaikanal, 27.09.1956, J. Pallithanam 2489; Ibid., 14.08.1967, K.M. Matthew \& K.T. Matthew 90188 (RHT); Madurai district, Hospital valley, s.d., V.Lakshmanan 87692; Kuthanatchi R.F., 25.05.1989, $V$. Lakshmanan 87692 (BSID); Nilgiri district, s.loc., 09.1883, J.S. Gamble 12477 (CAL); s.loc., 30.08.1983, K.N. Subramanian 9538 (FRC); DoddabettaKotagiri way, s.die., J. Remya \& Prasanna 73808: Ootty, 22.10.2015, A.K. Pradeep 86960 (CALI); upper Bhavani, s.d., J. Remya \& Prasanna 73762
1-2 mm long at sinus; lemmas 3-4 × 1-1.5 mm, oblong-lanceolate, 4-lobed, 3-nerved, slightly keeled, 3-awned, straight, scabrid, median awns 3$4.2 \mathrm{~mm}$ long, lateral awns $2.5-3 \mathrm{~mm}$ long, lateral lobes $0.5-1 \mathrm{~mm}$ long, acute-acuminate, hyaline; paleas 3-3.8 × 0.75-1 mm, elliptic-oblanceolate, hyaline, 2-keeled, ciliate above the half, coriaceous, acute or 2-lobed with a minute central notch at the apex; lodicules 2, 0.3-0.4 mm long, 3-toothed; stamens 3, anthers 1-1.2 mm long, oblong, filaments $0.5-0.75 \mathrm{~mm}$ long, slender, glabrous; ovary $0.3-0.5 \mathrm{~mm}$ long, obovate, styles 2, 0.5-0.75 $\mathrm{mm}$ long, slender, hyaline, stigma 1-2 mm long, plumose, creamy white. Caryopses $1.2-1.8 \times 0.2-$ $0.4 \mathrm{~mm}$, narrowly oblong-elliptic, dark brown.

Flowering \& fruiting: Flowering and fruiting from late August to December.

Habitat: Occurs in grasslands, soil pockets and in granitic rock crevices, and along Ghat road sides at higher elevations (900-2000 m), in association with Arundinella mesophylla Nees ex Steud., Chrysopogon nodulibarbis (both Poaceae), Henckelia humboldtiana (Gesneriaceae), Drosera indica L. (Droseraceae), Exacum wightianum Arn. (Gentianaceae), Impatiens acaulis (Balsaminaceae), Leucas ciliata Benth. (Lamiaceae), and Sonerila rheedei Wall. (Melastomataceae).

Distribution: India.

Specimens examined: INDIA, Karnataka, Bagalkot district, Badami fort, 09.1909, R.U. Shidi s.n. (BLAT); Chikmagalur district, Baba Budan hills, 18.10.1974, R.S. Raghavan 132420 (BSI); Ibid., 13.09.2014, K. Thoiba 137525; Kavikal Gandi hills, 13.09.2014, K. Thoiba 137503; way to Mullayangiri hills, 13.09.2014, K. Thoiba 137516a, 137516b, 137516c, 137516d (CALI). Kerala, Idukki district, Anamudi slopes, 19.11.1965, B.V. Shetty 26544 (CAL); Devikulam, 16.12.2014, K. Thoiba \& A.K. Pradeep 137591; Parunthpara, 16.10.2015, K. Thoiba \& A.K. Pradeep 146616, 146619; Peerumedu, 16.10.2015, K. Thoiba \& A.K. Pradeep 146631 (CALI); Kozhikode district, Vattappara, below Vellarimala, 15.12.1998, A.K. Pradeep 56232, 
(TBGT). Telangana, Mehbubnagar district, s.loc., 05.11.2008, B. Raviprasad Rao \& B. Sadasivaiah s.n. (BSID).

Tripogon bromoides Roem. \& Schult. var. longifolius Hook.f., Fl. Brit. India 7(22): 287. 1896. Tripogon festucoides Jaub. \& Spach. Ill. Pl. Orient. 4(34): 49, t. 333. 1851. Type: INDIA, Tamil Nadu, s.d., Perrottet 1260 (L [L0050269 digital image!]).

Tripogon lanatus Hochst. ex Steud., Syn. Pl. Glum. 1:301. 1854. Type: INDIA, Tamil Nadu, Montibus Niligiri, 1851, R.F. Hohenacker 922 (P [P00740185 digital image!]).

Figs. 11-13

Caespitose perennials. Culms 5-30 cm high, erect; nodes glabrous, slightly geniculate. Leaf sheaths 1$4 \mathrm{~cm}$ long, linear, fibrous, glabrous; ligules membranous with a tuft of 1-2 $\mathrm{mm}$ long villous hairs at apex; leaf blades 3-14 × 0.15-0.2 cm, linearlanceolate, flat-convolute, scabridulous, sparsely

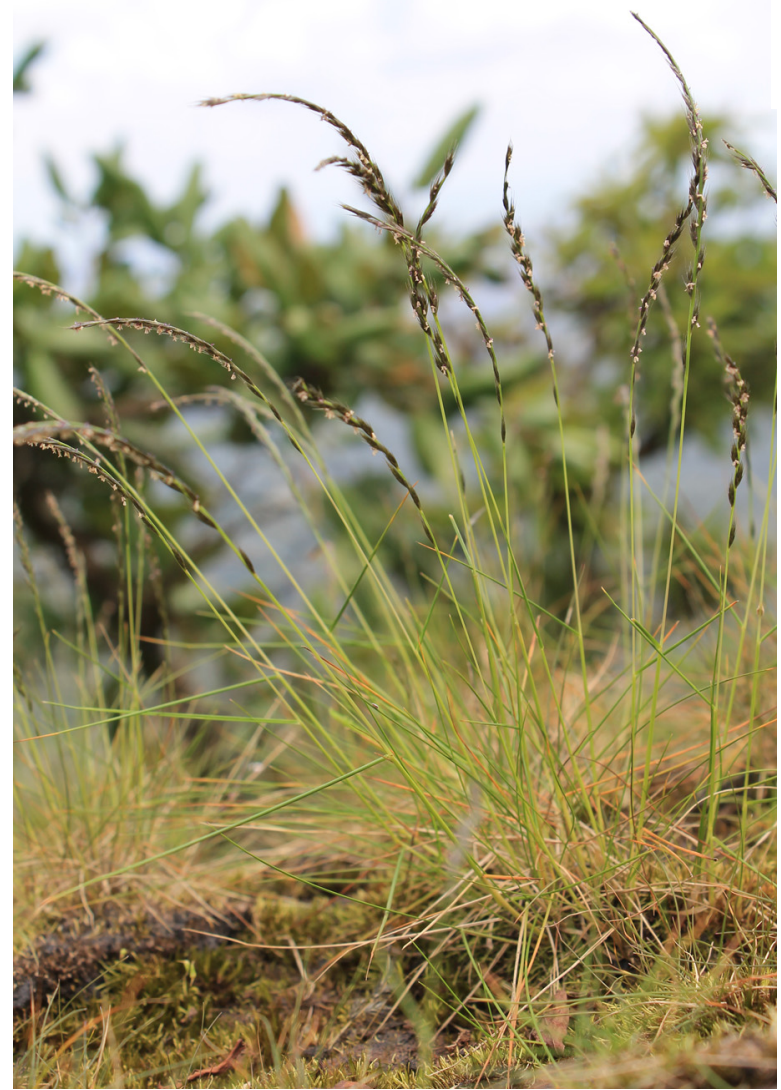

Fig. 11. Tripogon bromoides Roem. \& Schult. var. Iongifolius Hook.f. in its natural habitat (Photo by K. Thoiba).

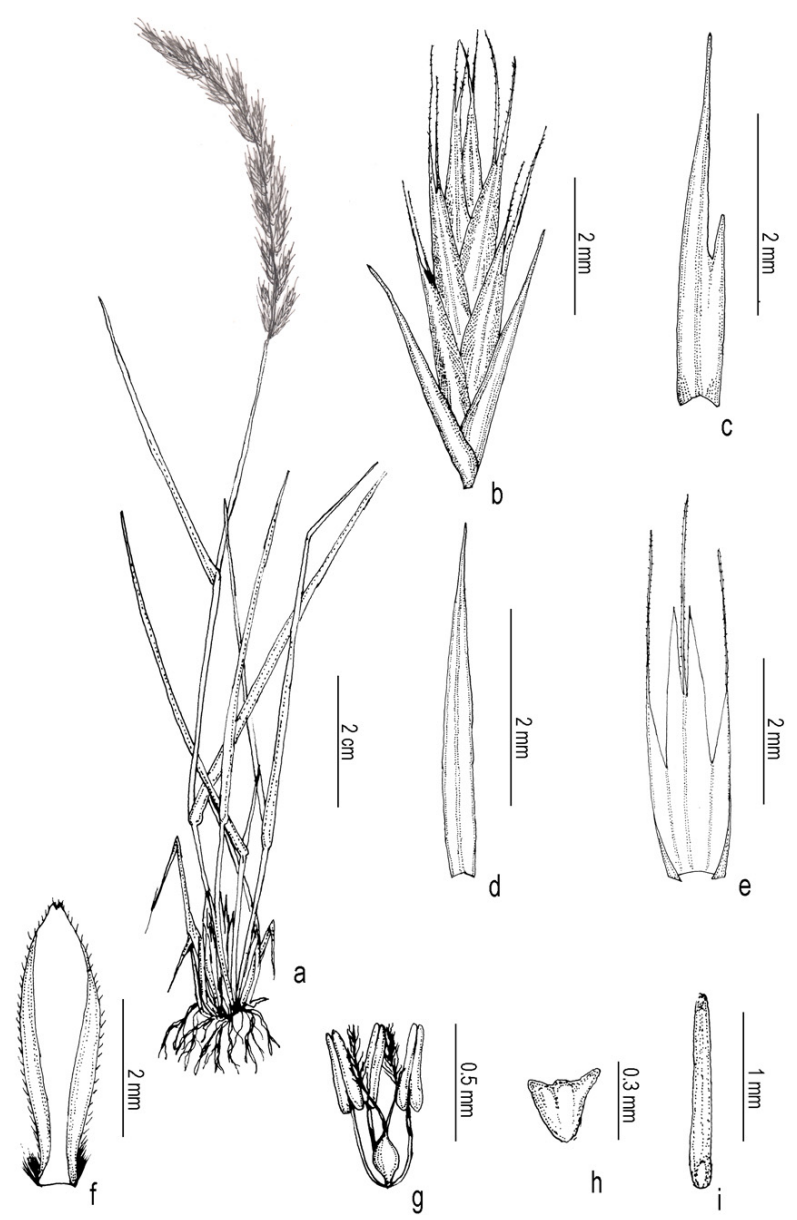

Fig. 12. Tripogon bromoides Roem. \& Schult. var. Iongifolius Hook.f.: a. Habit; b. Spikelet; c. Lower glume; d. Upper glume; e. Lemma; f. Palea; g. Stamen \& pistil; h. Lodicule; i. Caryopsis.

villous adaxially and glabrous abaxially, margins entire, acuminate at apex. Racemes 5-10 cm long, slender, straight to curved, 10-15 spikelets appressed in rachis; rachis $2.5-3.5 \mathrm{~mm}$ long, stout, triquetrous, glabrous or scabridulous; peduncles 5$10 \mathrm{~cm}$ long, glabrous. Spikelets $4.8-12 \times 2.5-3 \mathrm{~mm}$, ovate-lanceolate, compact, dorsiventrally flattened, dark green, 4-10-flowered; callus bearded, hairs 1$1.8 \mathrm{~mm}$ long; rachilla $0.75-1.2 \mathrm{~mm}$ long, glabrous to scabrid, almost straight, not persistent; lower glumes $3.3-4 \times 0.5-0.75 \mathrm{~mm}$, elliptic-lanceolate, notched on one-side, about 2-2.5 mm long from base, 1-keeled, 1-veined, coriaceous, apex acuminate; upper glumes $2.9-5 \times 0.5-0.75 \mathrm{~mm}$, elliptic-lanceolate, coriaceous, 1-keeled, 1-veined, 1-awned, awn continuous with apex, scabridulose, 


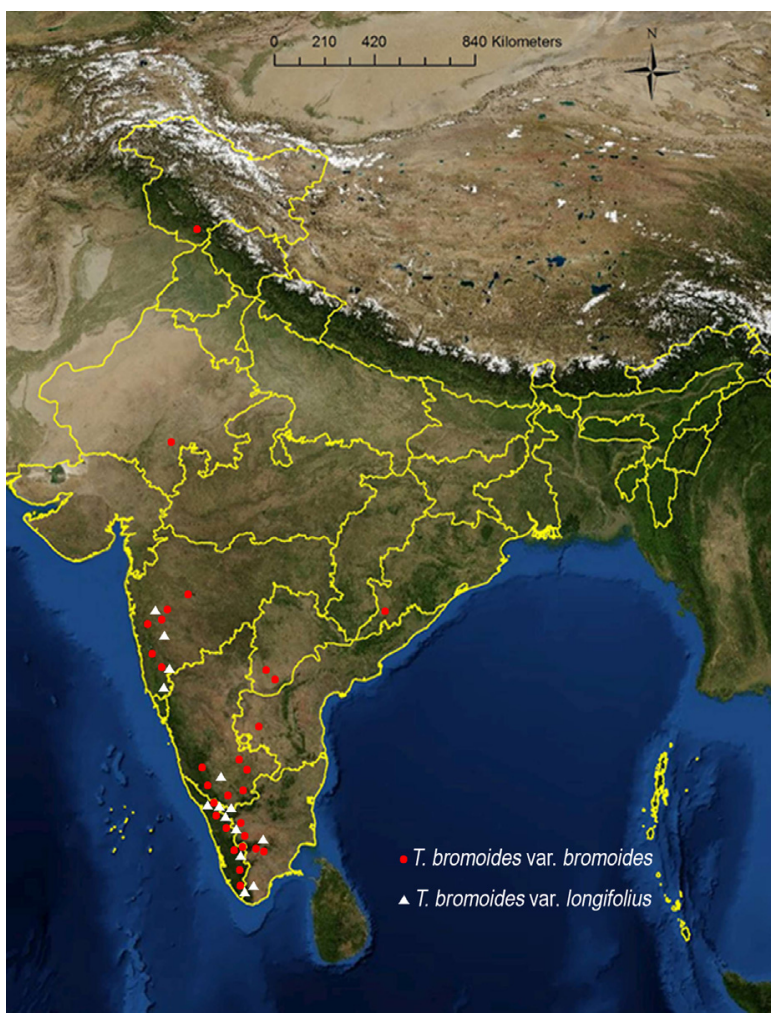

Fig. 13. Distribution of Tripogon bromoides Roem. \& Schult. var. bromoides and T. bromoides var. longifolius Hook.f. in India [drawn using ESRI (2011) ArcGIS Desktop: Release 10].

56423 (CALI); Thiruvananthapuram district, Agasthyamala, 14.10.1988, N. Mohanan 4279, 4300; Pongalapara, 28.08.1990, N. Mohanan 10050 (TBGT); Wayanad district, Chambra hills, 22.08.2013, K. Thoiba 134431 (CALI). Maharashtra, Kolhapur district, Gaganbawada, 03.10.1989, C.B. Salunkae 7409; Halkarni College, 08.07.2001, s.coll.s.n.; Vishalgad, 26.09.2008, K.V.C. Gosavi 2915 (SUK); Pune district, Purandar fort, 07.09.1907, R.K. Bhide 2071183; Raireshwar, 08.11.1958, V.D. Vartak 14633 (BSI); Ratnagiri district, Durgawadi, 24.09.2004, Aparna Watve 0412 (AHMA); Satara district, Kas, 09.08.2004, Aparna Watve 0211 (AHMA); Kas plateau, 19.09.2009, M.M. Lekhak 238; Mahavashi, 10.09.2005, Aparna Watve 0622 (SUK); Yavatmal district, Junnar, Ralegaon hill range, 23.09.1965, K. Hemadri 107267 (BSI). Tamil Nadu, Coimbatore district, Valparai, 28.07.1978, M. Chandrabose 57709 (CAL); Dindigul district, Kodaikanal, 16.09.1956, J. Pallithanam 2210;
Konalar, 09.09.1985, K.M. Matthew, S.J. Britto \& A. Usha 42331; Mannavanur lake exit, 30.08.1987, K.M. Matthew \& K.T. Matthew 50599; Perumalmalai, 30.10.1985, K.M. Matthew \& N. Rajendren 42442; Shembaganur, s.die., K.M. Matthew 741; Ibid., 01.06.1984, K.M. Matthew \& S.J. Britto 40231; Silver cascade top, 31.07.1985, S.J. Britto 41574; Vandaravu-Marion shola levinge path, 25.10.1988, K.M. Matthew 53644; VadakavunchiPerumal peak, 14.12.1989, K.T. Matthew \& S. Sebastian 53855; Vembadi peak, 04.11.1987, K.M. Matthew \& K.T. Matthew 51039 (RHT); Nilgiri district, Coonor peak, 06.1883, J.S. Gamble 15319; Kotagiri, Aravenu, 24.10.1956, K. Subramanyam 1109 (RHT); Coonor, Udagamandalam, 01.06.2014, Thoiba K. 138077 (CALI); Otacamund, Ebbanad, 10.09.1970, G.V.Subba Rao 36627 (MH); Pykara, 06.1883, J.S. Gamble 11835(RHT); Sholur, Gudallur-Ootty road, 22.10.2015, A.K. Pradeep \& K. Thoiba 86964, 86965; valley view, CoonorOotty road, 22.10.2015, A.K. Pradeep \& Thoiba K. 86961 (CALI); Tirunelveli district, 01.09.1964, A.N. Henry 17411 (CAL); Naduvattam, 23.06.2016, Nikhil Krishna \& K. Thoiba 146773, 146775 (CALI).

Notes: It differs from the typical variety by its short habit, dark green spikelets with sub-equal lemma awns, and lateral lobes of lemma being acute to acuminate at apex.

Tripogon capillatus Jaub. \& Spach, Ill. Pl. Orient. 4(33): 47, t. 332. 1851; Hook.f., Fl. Brit. India 7(22): 285. 1896; C.E.C.Fisch. in Gamble, Fl. Madras 3: 1834. 1934; Bor, Grass. Burma Ceylon India \& Pakistan 521. 1960; Sreek. \& V.J.Nair, Fl. Kerala Grass. 401. t. 83. 1991; S.Moulik, Grass. Bam. India 2: 622. 1997; Sasidh., Biodiv. Doc. Kerala-Fl. Pl. 595. 2004; Potdar, Salunkhe \& S.R.Yadav, Grass. Maharashtra 557. 2012. Type: INDIA, Western Ghats; from the Concan to the Nilghiri hills, s.d., Jacquemont s.n. (iso K [K000245009 digital image!], P [P00740179 digital image!]).

Figs. $14 \& 16$

Caespitose annuals, sometimes epiphytic. Culms 15-45 cm high, erect; nodes glabrous. Leaf sheaths 2-10 cm long, linear, glabrous; ligules membranous with a tuft of 1-2 mm long hairs on the margins; 


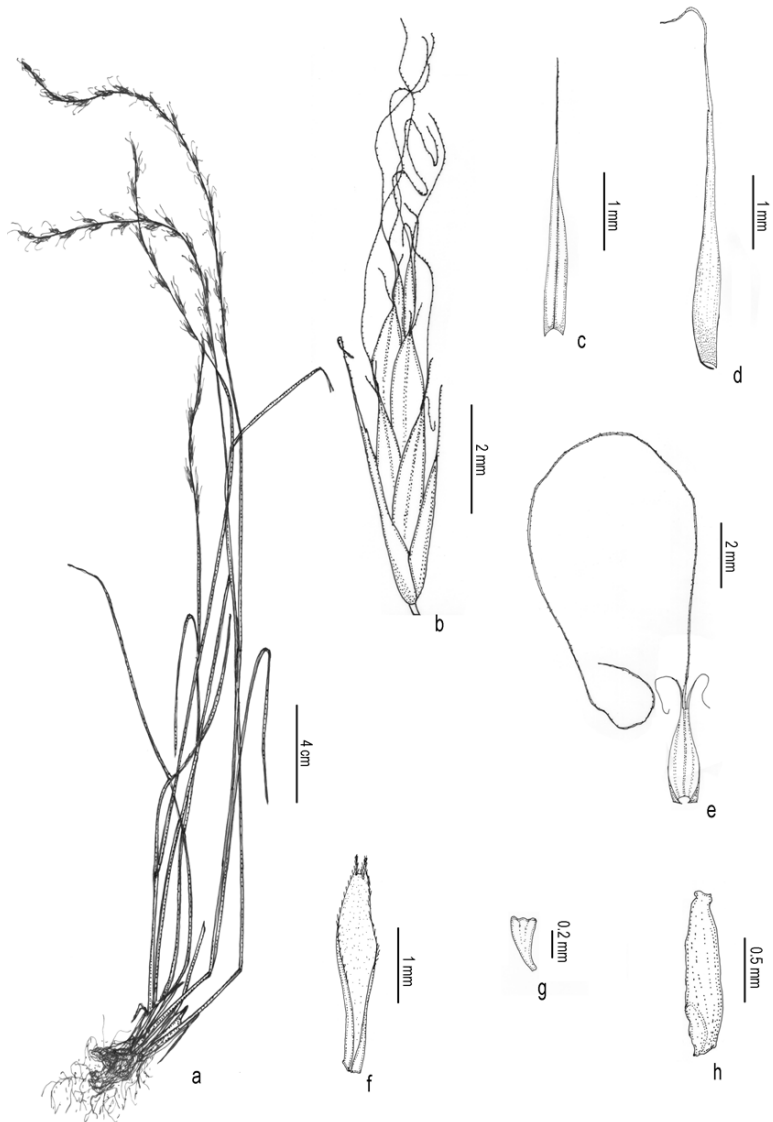

Fig. 14. Tripogon capillatus Jaub. \& Spach: a. Habit; b. Spikelet; c. Lower glume; d. Upper glume; e. Lemma; f. Palea; g. Lodicule; h. Caryopsis.

leaf blades $5-30 \times 0.1-0.2 \mathrm{~cm}$, linear-lanceolate, midrib prominent, acuminate to attenuate at apex. Racemes 10-30 cm long, filiform and flexuous, distant and loosely arranged spikelets; rachis 0.5$1.2 \mathrm{~cm}$ long, flexuous, smooth, triquetrous; peduncles 5-10 cm long, glabrous. Spikelets 5-8 × 1.3-1.6 mm long, linear-lanceolate, dorsiventrally flattened, 3-5-flowered; callus bearded, hairs 1-1.2 $\mathrm{mm}$ long; rachilla 1.2-1.6 mm long, not persistent, disarticulating with florets, glabrous; lower glumes $1.3-3.5 \times 0.5-0.75 \mathrm{~mm}$, elliptic-lanceolate, notched on one-side, 1-keeled, 1-nerved, attenuate or shortly awned at apex, awns 0.5-1.5 mm long, scabrid; upper glumes 3-3.5 × 0.4-0.75 mm, elliptic-lanceolate, 1-keeled, 1-nerved, apex awned, awns 5-6 mm long, scabrid, bristle-like; lemmas 2.8-4.5 × 0.4-1.2 mm (excluding awn), oblonglanceolate, 3-veined, 3-awned, awns scabrid, straight-geniculate, flexuous or filiform, lateral awns 2-8 mm long, median awns 15-35 mm long; paleas 2-2.8 × 0.4-1 mm, elliptic-oblanceolate, hyaline, 2-keeled, ciliate along the margins, 2-lobed at the apex; lodicules 2, 0.4-0.5 × 0.25-0.3 mm, truncate; stamens 3 , anthers 1-1.5 mm long, oblong, filaments $0.5-0.75 \mathrm{~mm}$ long, slender, glabrous; ovary $0.3-0.5 \mathrm{~mm}$, obovate styles $2,0.5-$ $0.75 \mathrm{~mm}$ long, slender, hyaline, stigma $0.75-1.2$ $\mathrm{mm}$ long, plumose, creamy white. Caryopses 1.3$1.6 \times 0.2-0.3 \mathrm{~mm}$, elliptic-oblong, dark brown.

Flowering \& fruiting: Flowering and fruiting from late August to early December.

Habitat: Occasional at higher elevations (500-1000 $\mathrm{m})$ on tree trunks, rocks and on dilapidated walls, in association with Aeschynanthus perrottetii A.DC. (Gesneriaceae), Eriocaulon sp. (Eriocaulaceae), Impatiens theuerkaufiana Ratheesh \& Sivad. (Balasaminaceae), Indopoa paupercula (Stapf) Bor, Arthraxon lancifolius Hochst. (both Poaceae), Porella sp. (Porellaceae), moss and fern species.

Distribution: India and Sri Lanka.

Specimens examined: INDIA, Goa, North Goa district, Surla plateau, Salan, 02.10.2005, Harshala Gad \& M.K. Janarthanam 243 (Goa University Herbarium). Karnataka, Chikmagalur district, Hazari, 1883, s.coll. 33677 (CAL); Kalhatti Falls, 19.10.1974, R.S. Raghavan 132501; s.loc., 09.1915, W.A. Talbot 2621 (BSI); s.loc., s.die., Hooker 539250; s.loc., s.die., W.M.Munro 866 (CAL); Kodagu district, Irippu falls, 01.01.2017, Nikhil Krishna \& K. Thoiba 146766 (CALI); Virajpet, 07.10.2010, Remya J. \& Prasanna 69556 (TBGT). Kerala, Kasargod district, Ranipuram, 20.09.2015, K. Thoiba 144164 (CALI); Palakkad district, Sirendhri, Nilgiri Biosphere Reserve, 06.11.2012, J. Remya \& Prasanna 74586 (TBGT); Wayanad district, Thirunelli, 09.10.2012, J. Remya 73861 (TBGT). Jharkhand, Giridih district, Parasnath, 01.10.1873, s.coll. 20777 (CAL). Maharashtra, Kolhapur district, Panhala, 10.10.2001, s.coll., s.n.; Amboli Ghat, 13.09.2008, K.V.C. Gosavi 2895 (SUK); Ibid., 08.11.2016, Nikhil Krishna \& K. Thoiba 146732 (CALI); Mumbai 
district, Trombay, 09.1907, A. Meebold 8847 (CAL); Borivali National Park, 28.08.1952, Fernandez 330 (BLAT); Nashik district, Igatpuri, Mahalunga hills, 13.09.1962, R.S. Rao 81381 (BSI, CAL); Pune district, Dhak hill range, Junnar, 29.09.1965, $K$. Hemadri 107454 (BSI, CAL); Dukar Ghat, Panchai, A.L. Nager, 24.09.1970, B.M. Wadhwa 127525; Durga Khilla, 06.11.1969, K. Hemadri 117916; Hari Chandnagarh, 25.09.1970, B.M. Wadhwa 127621; Khandala, 20.09.1902, G.A. Gammie 15413; Ibid., 09.1910, L.D. Earade s.n. (BSI); Khubi, 16.09.2005, S.B. Nagarkar s.n. (AHMA); Lonavala-Bridgeon dam, 05.09.1956, S.K. Jain 5996; Muhshi, Ambawane, 07.09.1964, B. Venkata Reddi 99093 (BSI); Rajgad, 25.09.1956, V.D. Vartak 5999 (AHMA); Raireshwar, 18.08.1958, V.D. Vartak 8 (CAL); way to INS Shivaji-Lonavle, 27.04.1964, B. Venkata Reddi 98739 (BSI); Tork range, 19.11.1968, K. Hemadri 115773 (CAL); Raigad district, Dhak, 07.2007, S.S. Rahangdale s.n. (AHMA); Satara district, Mahabalaeshwar, 29.09.2013, K.M. Manudev 134467 (CALI); Yawateshwar, 01.09.1991, C.B. Salunkhe 8223 (SUK); Sindhudurg district, Amboli Ghat, 11.10.1970, B.G. Kulkerni 121622 (BSI); Ibid., 30.04.2001, s.coll. s.n. (SUK); Thanae district, Cactle rock, 10.1908, A. Meebold 10501; Durgadi killa, 20.09.1968, K. Hemadri 117916; Panecnai plateau, Taroda range, 19.11.1968, K.V. Billore 115773; near Kirouli School, 16.09.1962, R.S. Rao 81563(CAL).

Notes. The median awns of the lemma are flexuous and reach up to $35 \mathrm{~mm}$.

Tripogon filiformis Nees ex Steud., Pl. Glum. 1: 301. 1854; Hook.f., Fl. Brit. India 7(22): 288. 1896; Bor, Grass. Burma Ceylon India \& Pakistan 521. 1960; S.Moulik, Grass. Bam. India 2: 622. 1997; S.M.Philip \& S.L.Chen, Kew Bull. 57(4): 919. 2002; Potdar, Salunkhe \& S.R.Yadav, Grass. Maharashtra: 560. 2012. Type: INDIA, Northwest India, s.d., Royle s.n. (iso K [K000245038 digital image!]).

Plagiolytrum filiforme Nees, Proc. Linn. Soc. London 1: 95. 1841. nom. nud.

Plagiolytrum unidentatum Nees, Proc. Linn. Soc. London 1: 95. 1841. nom. nud.
Catapodium filiforme Nees ex Duthie, N.W. Ind. 33. 1883. nom. inval.

Tripogon semitruncatus Nees ex Steud., Pl. Glum. 1: 301. 1854. Type: INDONESIA, Monte Laeou Javae, s.d., Junghugn, s.n. (iso L [L0050285 digital image!]).

Tripogon unidentatus Nees, Pl. Glum. 1: 301. 1854. Type: Northwest India, s.d., Royle P. 92 (holo P; iso $\mathrm{K})$.

Tripogon filiformis Steud. var. tenuispicus Hook.f., Fl. Brit. India 7(22): 288. 1896. Type: Northeast India, s.d., Wallich 8892 (holo K [K000245041 digital image!]).

Tripogon nanus Keng f., Acta. Bot. Sin. 9: 71. 1960. Type: CHINA, Sikang, Miao-niu, July 1940, K.L. Chu 7683 (holo N).

Figs. $15 \& 16$

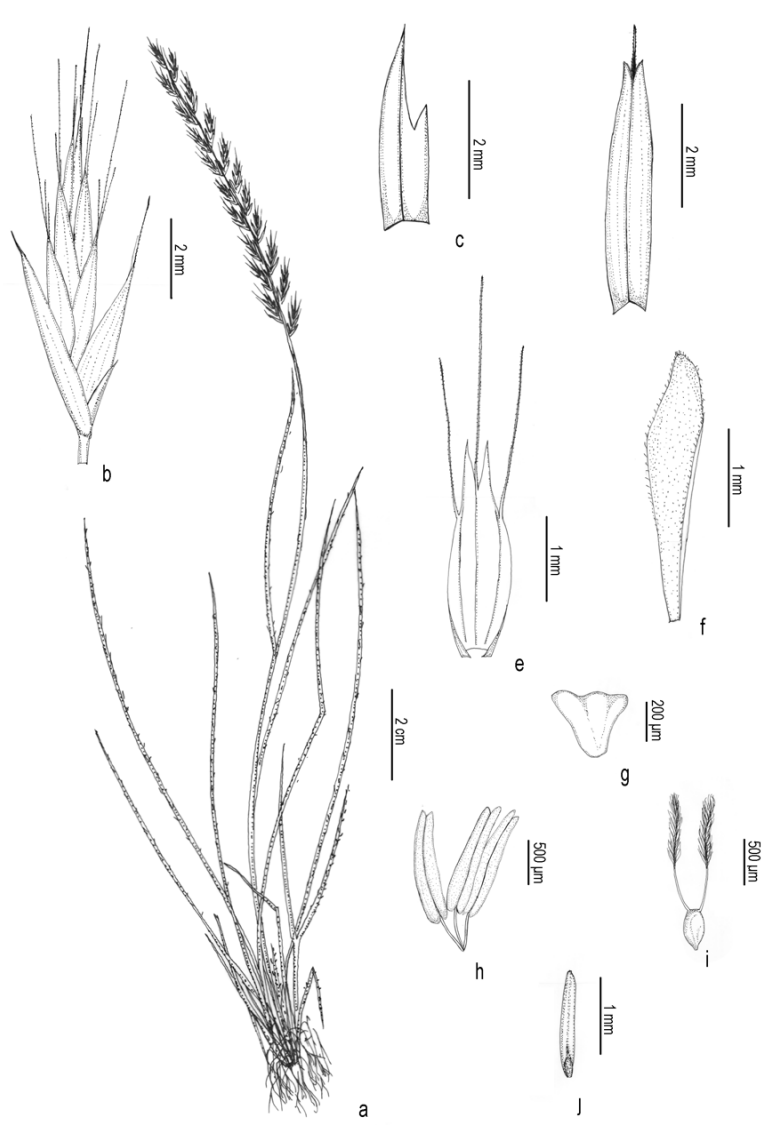

Fig. 15. Tripogon filiformis Nees ex Steud.: a. Habit; b. Spikelet; c. Lower glume; d. Upper glume; e. Lemma; f. Palea; g. Lodicule; h. Stamen; i. Pistil; j. Caryopsis. 
Caespitose perennials. Culms 10-50 cm high, erect, slender; nodes glabrous. Leaf sheaths $2-10 \mathrm{~cm}$ long, linear, ribbed and hairy; ligules obsolete, ciliate with a tuft of 1-2 mm long hairs at both apex; leaf blades 5-17 × 0.2-2.5 cm, linear-lanceolate, filiform, convolute, more hairy towards collar, densely pilose along both surfaces, acuminate to attenuate at apex. Racemes 5-25 × 0.3-0.5 cm, 25-35 spikelets loosely or tightly appressed to concavities in rachis; rachis 3-5 mm long, stout and slightly scabrid; peduncles 3-10 cm long, glabrous. Spikelets 6-8 × 1-2 mm long, linear, dorsi-ventrally flattened, 4-10flowered; callus bearded, hairs $0.5-1 \mathrm{~mm}$ long; rachilla $0.5-0.75 \mathrm{~mm}$ long, glabrous, slightly zigzag; lower glumes 1.5-2.5 × 0.5-1 mm, ovatelanceolate, asymmetrical, notched on one side, 1keeled, 1-nerved, keels slightly scabrid, margins serrulate, obtuse at apex; upper glumes 2.5-4 × 0.5$1 \mathrm{~mm}$, elliptic-lanceolate, 1-keeled, 1-nerved, apex dentate, 1-awned, awns 1-1.2 mm long at sinus; lemmas 5-8 × 1-1.5 mm (including awn), oblonglanceolate, 4-lobed, 3-awned, 3-nerved, slightly keeled, 3-awned, awns scabrid, straight or flexuous, median awns 2.5-5 mm long, lateral lobes 1-1.2 mm long, lanceolate, apex acute, margins serrulate, lateral awns 1-2 mm long, arising from sinus; paleas $2-3 \times 0.5-0.75 \mathrm{~mm}$, elliptic-lanceolate, slightly winged, 2-nerved, 2-keeled, hyaline, keels ciliate more at the tip, apex obtuse or minutely bimucronate; lodicules 2, 0.3-0.4 mm long, truncate; stamens 3, anthers 1-1.5 mm long, oblong; filaments $0.5-0.75 \mathrm{~mm}$ long, slender, glabrous; ovary $0.3-0.5 \mathrm{~mm}$, obovate, styles $2,0.5-0.75 \mathrm{~mm}$ long, slender, hyaline, stigma 0.75-1.2 mm long, plumose, creamy white. Caryopses $1.5-2.5 \times 0.3-$ $0.5 \mathrm{~mm}$, narrowly oblong-elliptic, light brown.

Flowering \& fruiting: Flowering and fruiting from August to November.

Habitat: Moist rock outcrops at higher elevation grassland (1000-4500 m). It grows in association with Cyanotis sp. (Commelinaceae), Eriocaulon sp. (Eriocaulaceae), Impatiens goughii Wight (Balasaminaceae), Tripogon bromoides, $T$. karnatakensis (both Poaceae), and moss species.

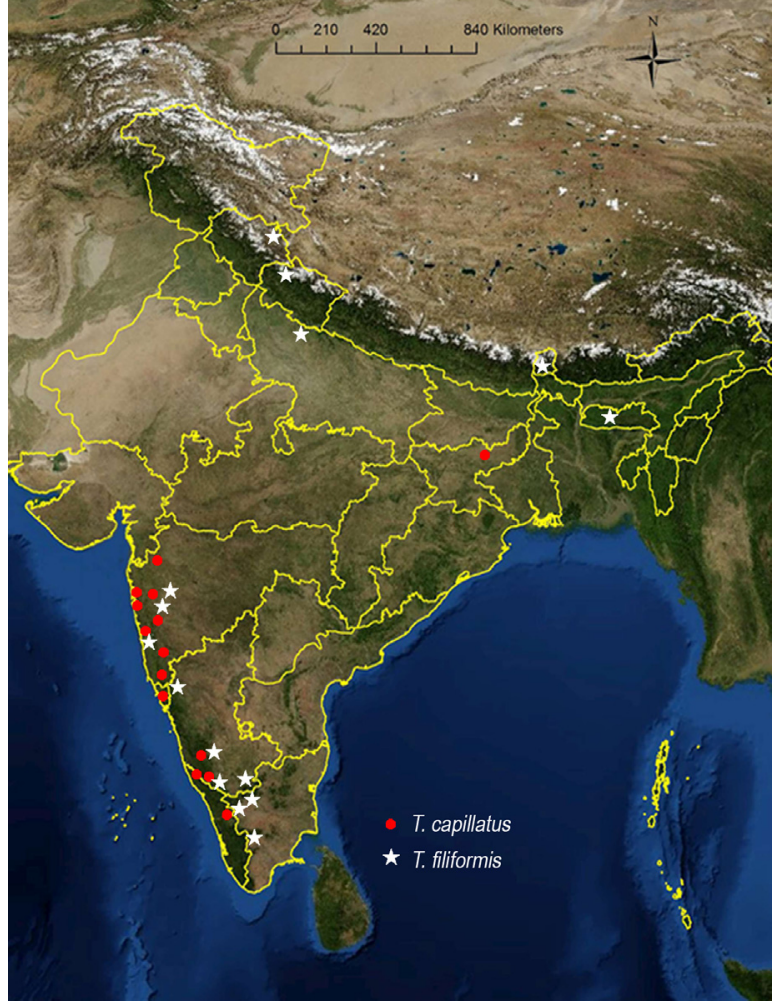

Fig. 16. Distribution of $T$. capillatus Jaub. \& Spach and T. filiformis Nees ex Steud. in India [drawn using ESRI (2011) ArcGIS Desktop: Release 10].

Distribution: India, Bhutan, Nepal, North Myanmar, Pakistan and China.

Specimens examined: INDIA, Karnataka, Belgaum district, s.loc., 10.1897, W.A. Talbot s.n. (BSI); Chamarajanagar district, Biligirirangan Betta, 28.04.1962, A.S. Rao 80409 (CAL); Chikmagalur district, Kavikal Gandi hills, 13.09.2014, K. Thoiba 137504 (CALI); Kodagu district, Tadiyandamol hills, 26.11.2014, K. Thoiba 137555 (CALI). Himachal Pradesh, Lahaul \& Spiti district, Shell village site, 13.02.1957, S.C. Agarwal 1145 (CAL). Maharashtra, Pune district, Khandala, Paoli hill, 17.09.1951, H. Santapau 13409, 13415 (BLAT); Junnar, Ambe plateau, 04.10.1965, K. Hemadri 107590; Khed Taluk, near Gayimukh, 07.10.1962, K.P. Janardhanan 81643 (BSI); Raigad district, Matheran, hart point, 17.10.1960, N.A. Irani 5567 (BLAT). Meghalaya, East Khasi hills district, K \& J hills, 25.09.1956, G. Panigrahi 3498 (CAL). Sikkim, North Sikkim district, Lachung, 01.09.1892, G.A. Gammie 1095; s.loc., 16.10.1875, 
s.coll. 25326; s.loc., 08.1866, Wight s.n. (CAL). Tamil Nadu, Coimbatore district, Velliangiri hills, 30.09.2016, A.K. Pradeep, Nikhil Krishna \& K. Thoiba 146721, 146724, 146730 (CALI); Dindigul district, Kodaikanal, Mannavanur, Sheep farm, 28.08.1987, K.M. Matthew 5044; Kukkal, 19.10.1987, K.M. Matthew 50798; Vembadi peak, 17.12.1989, K.M. Matthew \& K.T. Mathew 53998 (RHT); Salem district, Shevaroy hills, 10.08.2004, A.K. Pradeep 93156a, 93156b, 93156c, 10 93156d, 93156e (CALI). Uttarakhand, Pauri Garhwal district, Chopra, 21.09.1975, A.S. Rao 56419 (CAL). Uttar Pradesh, s.loc., 10.1890, J.F. Duthie 10774 (CAL). 'Himalayas', s.die., s.coll. s.n. (MH).

Notes: Tripogon filiformis is a high altitude (1000$4500 \mathrm{~m}$ ) species, often confused with T. bromoides and $T$. longearistatus but can be distinguished by its densely villous leaves, dense feathery racemes and long awned lemmas.

Tripogon idukkianus Sunil \& Pradeep, Phytotaxa 202(4): 295. 2015. Type: INDIA, Kerala, Idukki district, Ramakkalmedu 1500 m, 10.11.2002, C.N. Sunil 2267 (holo K; iso CAL!, CALI [CALI112100!], $\mathrm{MH}[\mathrm{MH} 00003234 !])$.

Figs. $17,18 \& 22$

Caespitose perennials. Culms up to $55 \mathrm{~cm}$ high, slender; nodes glabrous. Leaf sheaths 5-9 cm long, coriaceous, sparsely hispid; ligules with a tuft of hairs, hairs 1-2 mm long, basal leaf sheaths papery; leaf blades $20-62 \times 0.2-0.3 \mathrm{~cm}$, linear-lanceolate, flat or involute, glabrous or sparsely hairy on both surfaces, midrib inconspicuous, margins entire, acuminate. Racemes 15-45 cm long, 20-58 spikelets loosely or tightly arranged in rachis; rachis smooth, angular, glabrous. Spikelets 5-13 × 1.5$2.5 \mathrm{~mm}, 6-14-f l o w e r e d$, secund, pale-green or yellow, distant, laterally compressed, florets loosely imbricate; callus bearded; rachilla usually not visible; lower glumes 2-3 × 1-1.5 mm, 1-veined, membranous, asymmetrical, and broadened on one-side below the middle, apex sub-acute, sometimes lobed; upper glumes $4-5 \times 1-1.5 \mathrm{~mm}$, lanceolate-oblong, 1-veined, membranous, apex

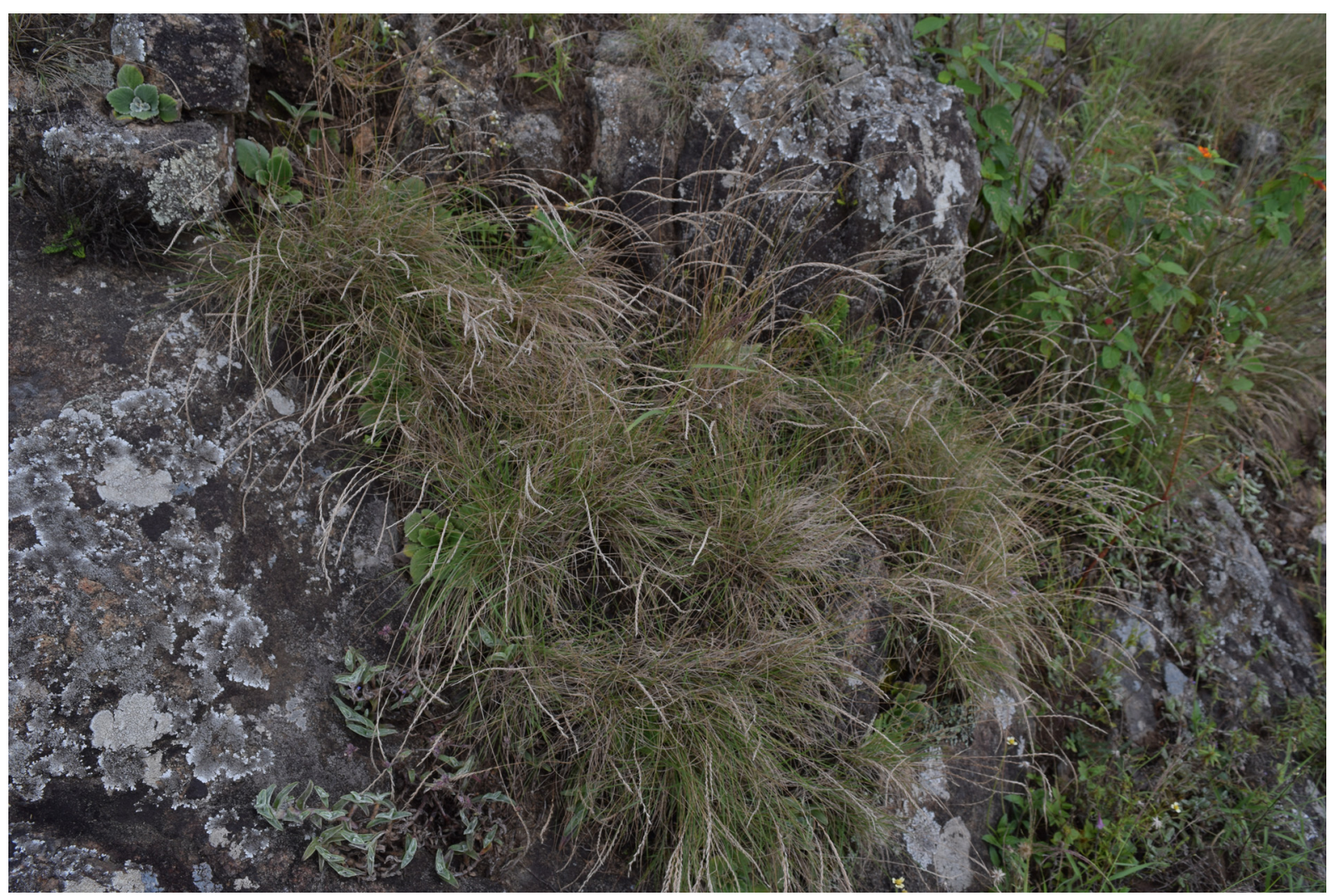

Fig. 17. Tripogon idukkianus Sunil \& Pradeep in its natural habitat at Ramakkelmedu in Idukki district (Photo by A.K. Pradeep). 


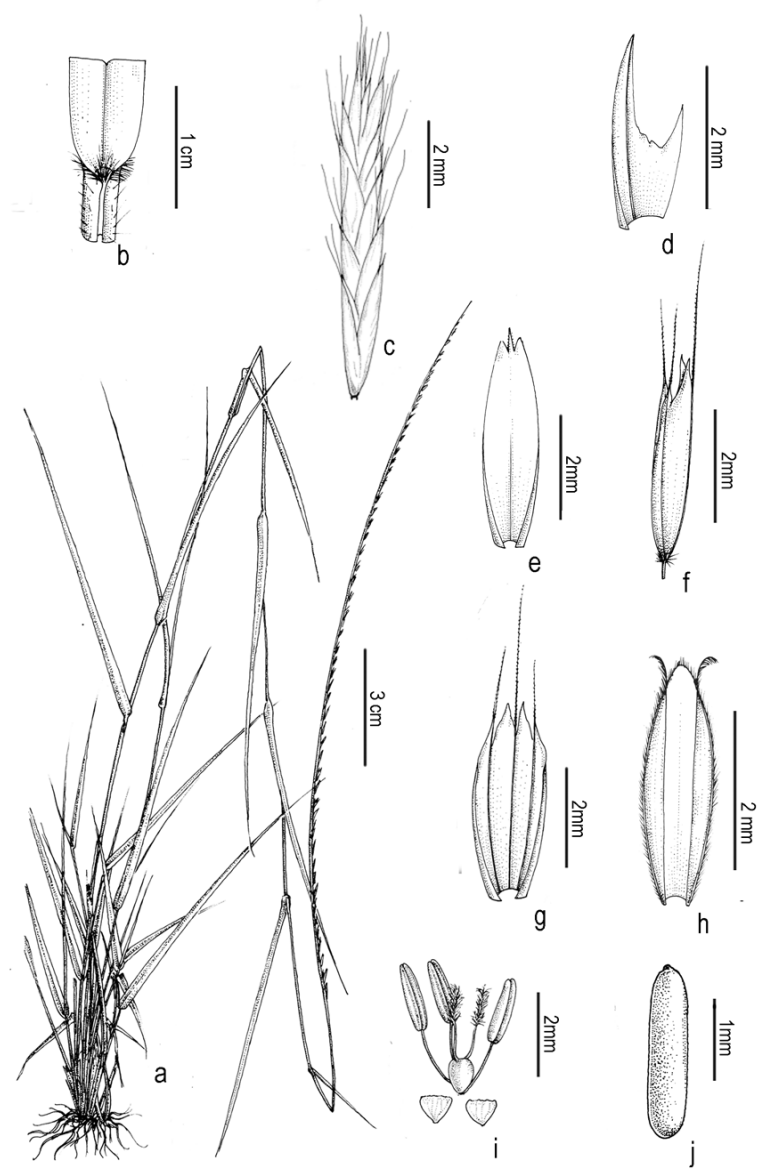

Fig. 18. Tripogon idukkianus Sunil \& Pradeep: a. Habit; b. Ligule; c. Spikelet; d. Lower glume; e. Upper glume; f. Floret; g. Lemma; h. Palea; i. Lodicule, stamen \& pistil; j. Caryopsis.

acute, notched, mucronate, mucro $0.5 \mathrm{~mm}$ long, scabrous, the lateral lobes as long or shorter than the mucro; lemmas $3-3.5 \times 2-2.5 \mathrm{~mm}$, membranous, grey-green, 3-veined, 3-awned, median awn 2-2.5 mm long, straight, rigid, scabridulous, lateral awns up to $2 \mathrm{~mm}$ long, apex acute, 4-lobed, each lobe, 0.5-1 ×0.25 mm, ovatelanceolate; paleas $2.5-3 \times$ c. $1 \mathrm{~mm}$, hyaline, obovate, apex obtuse or acute, bi-setaceous sub-apically, setae $0.25 \mathrm{~mm}$ long, curved, ciliolate; stamens 3 , anthers 1-1.5 mm long; ovary c. $0.25 \mathrm{~mm}$ long, globose, styles 2, stigma $0.75 \mathrm{~mm}$ long, purplish, feathery. Caryopses 1.5-1.6 × 0.25-0.3 mm, oblong-lanceolate, cylindric, light brown.

Flowering \& fruiting: Flowering and fruiting from October to late December.

Habitat: This species grows from 1000-1500 m elevations on seasonally wet rocky hillsides, in association with Apluda mutica L., Eragrostiella bifaria, Themeda tremula Hack., Tripogon bromoides (all Poaceae), Crotalaria beddomeana Thoth. \& A.A.Ansari (Fabaceae), Henckelia incana (Gesneriaceae) and Leucas ciliata (Lamiaceae).

Distribution: Endemic to South India, hitherto known only from the type locality.

Specimens examined: INDIA, Kerala, Idukki district, Ramakkalmedu, 1500 m, 13.11.2014, K. Thoiba \& A.K. Pradeep 137546; Ibid., 16.12.2014, K. Thoiba \& A.K. Pradeep 137584 (CALI).

Notes: Similar to Tripogon bromoides but can be identified by its sparsely hairy leaf blades, widely spread spikelets and paleas with an obtuse or acute apex with two sub-apical setae.

Tripogon jacquemontii Stapf, Kew Bull. 64: 85. 1892; Hook.f., Fl. Brit. India 7(22): 286. 1896; Bor, Grass. Burma Ceylon India \& Pakistan 522. 1960; S.Moulik, Grass. Bam. India 2: 622. 1997; Pull., Fl. Andhra Pradesh 3: 1268. 1997; Sasidh., Biodiv. Doc. Kerala-Fl. P1. 595. 2004; Potdar, Salunkhe \& S.R.Yadav, Grass. Maharashtra: 560. 2012. Lectotype (designated by Thoiba \& Pradeep, 2018): INDIA, Maharashtra, Poona, 09.02.1892, Jacquemont 320 (K [K000245018 digital image!]). Figs. 19, 20 \& 22

Tufted perennials. Culms 10-70 cm high, glaucous, terete, erect; nodes glabrous. Leaf sheaths $2-10 \mathrm{~cm}$ long, terete, glabrous; ligules ciliate, membraneous, with a tuft of 1-2 mm long hairs at apex; leaf blades 4-30 × 0.1-0.3 cm, linear, sub-filiform, involute, glaucous, setaceous, acuminate to attenuate at apex. Racemes 10-30 cm long, stout, erect or curved towards apex; 15-35 spikelets loosely arranged in rachis; rachis $3-12 \mathrm{~mm}$ long, glabrous or scabrid; peduncles $8-15 \mathrm{~cm}$ long, glabrous. Spikelets 7.5$16 \times 1-1.5 \mathrm{~mm}$, narrowly elliptic, linear, distant, leaden grey, 4-25-flowered; callus bearded, hairs c. $0.5 \mathrm{~mm}$ long; rachilla $0.3-0.4 \mathrm{~mm}$ long, glabrous, straight or zig zag, not persistent; lower glumes 2.8$3.5 \times 0.3-0.5 \mathrm{~mm}$, asymmetrical, notched on oneside, ovate-lanceolate, 1-nerved, 1-keeled, keels slightly scabrid, with acute apex; upper glumes 3$4 \times 0.5-0.8 \mathrm{~mm}$, elliptic-ovate, 1-nerved, 1-keeled, 


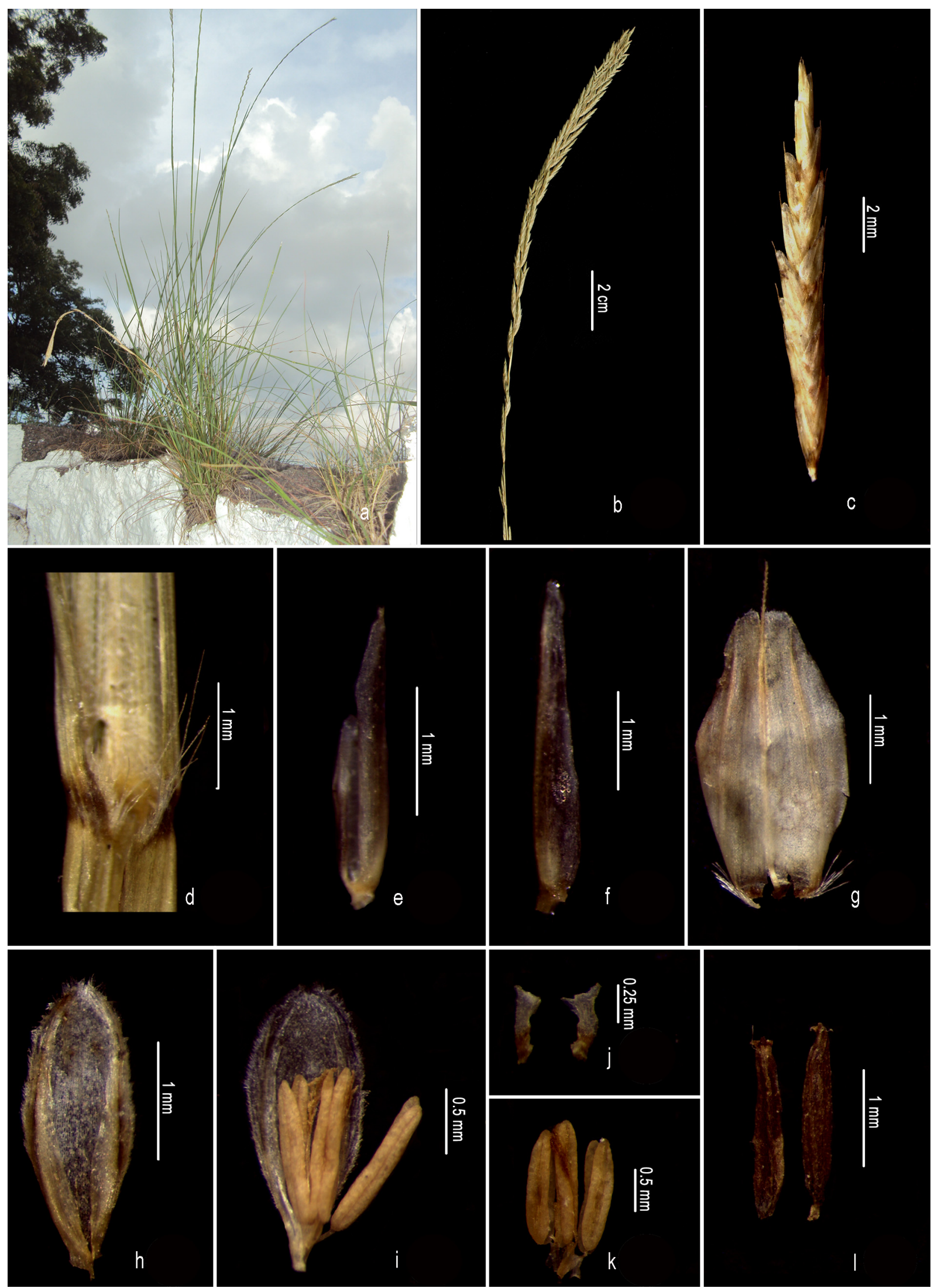

Fig. 19. Tripogon jacquemontii Stapf: a. Habit; b. Enlarged portion of raceme; c. Spikelet; d. Ligule; e. Lower glume; f. Upper glume; g. Lemma; h. Palea; i. Palea with flower; j. Lodicule; k. Stamen \& pistil; I. Caryopsis. 


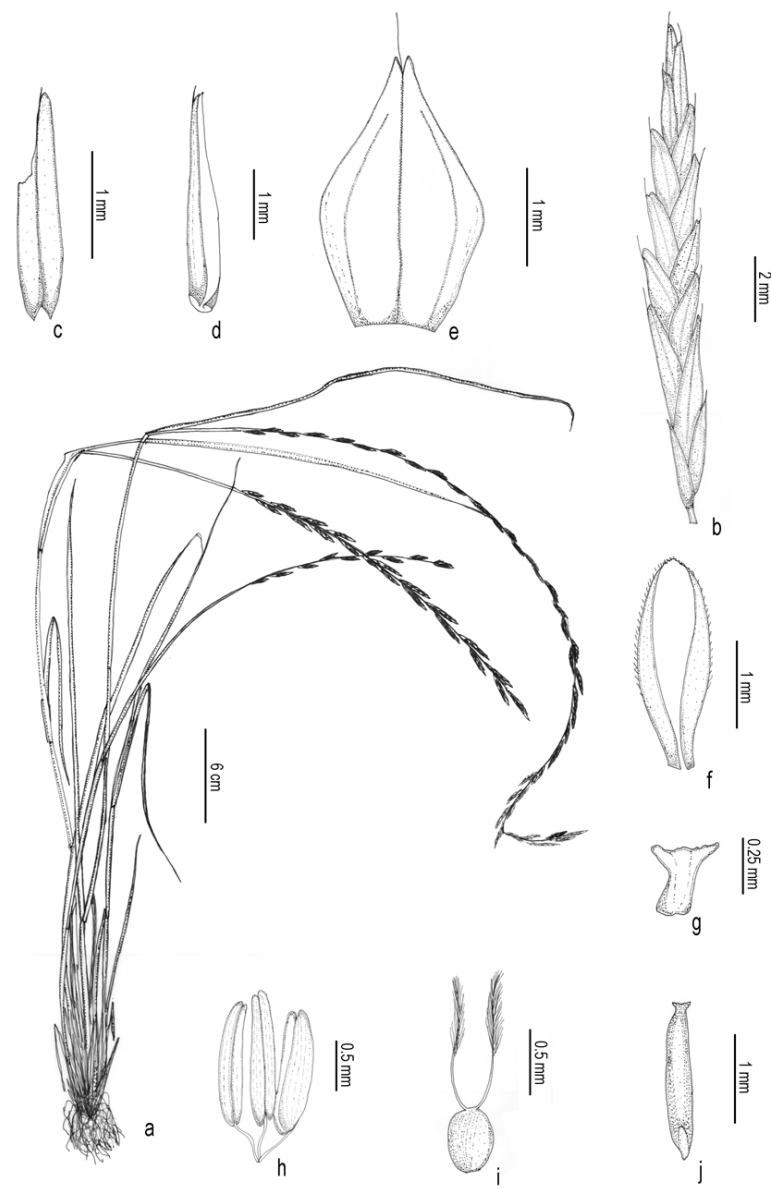

Fig. 20. Tripogon jacquemontii Stapf: a. Habit; b. Spikelet; c. Lower glume; d. Upper glume; e. Lemma; f. Palea; g. Lodicule; h. Stamen; i. Pistil; j. Caryopsis.

apex apiculate; lemmas 3-4 × 0.6-1 mm, broadly ovate, 2-lobed, 3-nerved, slightly keeled, 1-awned, awns 1-1.5 mm long, scabridulous, lateral lobes membranous; paleas $2.3-3 \times 0.5-0.8 \mathrm{~mm}$, elliptic, narrowly winged, hyaline, 2-keeled; keels scabrid, obtuse or emarginated at apex; lodicules 2, c. 0.3 mm long, slightly cordate; stamens 3 , anthers 1$1.6 \mathrm{~mm}$ long, oblong, filaments $0.5-0.75 \mathrm{~mm}$ long, slender, glabrous; ovary $0.3-0.5 \mathrm{~mm}$ long, obovate, styles 2, 0.5-0.75 mm long, slender, hyaline stigma 0.75-1.2 mm long, plumose, creamy white. Caryopses $1.4-1.6 \times 0.3-0.5 \mathrm{~mm}$, linear-oblong, light brown.

Flowering \& fruiting: Flowering and fruiting from August to early November.

Habitat: Gregarious, usually found in tussocks in open grassland, along boulders and on old walls of forts. It grows in association with Apluda mutica, Dimeria deccanensis Bor, Eragrostis maderaspatana Bor, E. minor Host, Eragrostiella bifaria, Tripogon bromoides (all Poaceae) and Pouzolzia zeylanica (L.) Benn. (Urticaceae).

\section{Distribution: India.}

Specimens examined: INDIA, Karnataka, Belgaum district, s.loc., 1897, Talbot 9782 (BLAT); Dharwad district, fort walls, 10.09.1890, W.A. Talbot 2301 (BLAT). Gujarat, Dhahod district, Devgadh, s.d., G.S. Puri 27303 (BSI). Jammu \& Kashmir, Kathua district, Bhullari, 20.09.1963, R.S. Rao 91987 (BSI). Madhya Pradesh, Indore district, Manpur in Mordu road, 18.10.1982, A.S. Rao 83808 (BSI). Maharashtra, Akola district, Narnala fort, 25.02.1978, S.Y. Kamble 152866 (BSI); Amravati district, Chikhladara, Melghat, 23.08.1976, M.Y. Ansari 143769 (BSI); Aurangabad district, Ajanta caves, 06.11.1961, R.S. Rao 71440 (BSI); Buldhana district, Lodi forest, 25.09.1982, P.G. Diwaker 164171 (BSI); Jalna district, Shelgaon, 31.10.1961, K.P. Janardhanan 72827 (CAL); Nagpur district, Amravati road-39/3 mile point, 17.09.1963, R.S. Rao \& U.R. Deshpande 91718a, 91718b (BSI); Nashik district, Peth Ghat, 08.08.1961, K.P. Janardhanam 71983 (CAL); Saptashrungi Hill, 15.08.1983, P.L. Narasimhan 165257 (BSI); Osmanabad district, Ramling hill, 06.10.2001, s.coll. s.n. (SUK); Pune district, AIT, 08.2007, S.S. Rahangdale s.n. (AHMA); Awhat-Shorewadi, on the way from Khed Taluk, 10.08.1961, K.P. Janardhanan 72406 (BSI); Barshinge hills 11.09.1966, R.D. Pataskar 101263 (BSI, CAL); Bopodi, 13.02.1902, s.coll. 15309; Bawjhan, 10.10.1956, Jain 7535; between mental hospital \& Vishrantwadi, 23.09.1960, M.Y. Ansari 64727; Chakan-Talegaon road, 19.09.1960, K.P. Janardhanan 66417; Camp canal cantt., 29.07.1960, K.C. Kanodia 64410; Donaje, 07.08.1964, M.Y. Ansari 97569 (BSI); Ganeshkhind, 08.1951, B.A. Razi 6276 (AHMA); Gargatwadi, 19.09.1960, K.P. Janardhanan 66276; Junnar, Shivneri hill, 14.08.1964, K. Hemadri 99655; Katraj Ghat, 30.09.1956, S.K. Jain 4076 (BSI); Katraj hill, 21.07.1956, V.D. Vartak 5249 (AHMA); 
Kharpudi hill top, 05.08.1961, K.P. Janardhanan 71835; Khed, Chakan-Talegaon road, $4^{\text {th }}$ mile point, 19.09.1960, K.P. Janardhanan 66417; Mahalunge, 02.11.1961, K.P. Janardhanan 72911; top of peth Ghat, 08.08.1961, K.P. Janardhanan 71983; Shelgaon, 31.10.1961, K.P. Janardhanan 72827; Manchar, Ghodegoan, 09.09.1956, G.S. Puri 7302; Markandey Top, 16.09.1966, R.D. Pataskar 108889; Narayangaon, Junnar, 23.10.1967, K. Hemadri 107055; Parvathi hills, 22.07.1960, M.Y. Ansari 64368 (BSI); Shivneri, 19.10.2001, s.coll.s.n. (SUK); Shivneri eastern exposure, 13.10.1962, s.coll. 83793; Shivneri hills, on way to top Junnar, 25.07.1963, M.Y. Ansari 88706; Shivneri fort, 10.10.1962, Hemadri \& R.S. Rao 83545; Ibid., 15.08.1964, K. Hemadri 99685; Sinhagad, s.d., G.S. Puri s.n.; Ibid., 09.08.1956, G.S. Puri 5675; Taleran, 09.2006, S.S. Rahangdale s.n.; Vetal hill, 08.10.1987, V.N. Jhoshi 333(AHMA); s.loc., 18.06.1902, L.D. Garade 486 (BSI); Raigad district, Shelu budruk, 29.08.1977, S.Y. Kamble 150213 (BSI). Rajasthan, Banswara district, s.loc., 20.03.1976, V. Singh 2913 (CAL); Chittorgarh district, s.loc., 17.08.1979, V. Singh \& R.P. Pandey 7249 (CAL). Tamil Nadu, Coimbatore district, Poonachi, 10.10.1901, C.A. Barber 3726 (MH). Telangana, Hyderabad district, Golconda fort, 13.09.2014, A.K. Pradeep \& K. Thoiba 144128a, 144128b, 144128 c, 144128d; Ibid., 07.02.2018, K. Thoiba 146795 (CALI).

Notes: Dey and Prasanna (2019) reduced T. polyanthus as a variety of $T$. jacquemontii, which is not accepted here, as our field studies showed both as two distinct species though they may look similar in herbarium specimens. Tripogon jacquemontii can easily be distinguished by its glaucous leaves and culms, and awns either shorter than the lemma or occasionally almost absent.

Tripogon karnatakensis Thoiba \& Pradeep, Phytotaxa 272(2): 126. 2016. Type: INDIA, Karnataka, Chikmagalur district, Kavikal Gandi hills, 1450 m, 13.09.2014, T. Kottekkattu \& M. Yoonus T137510 (holo CAL!; iso CALI [CALI1112092!], $\mathrm{MH}$ !, K!).

Figs. $21 \& 22$

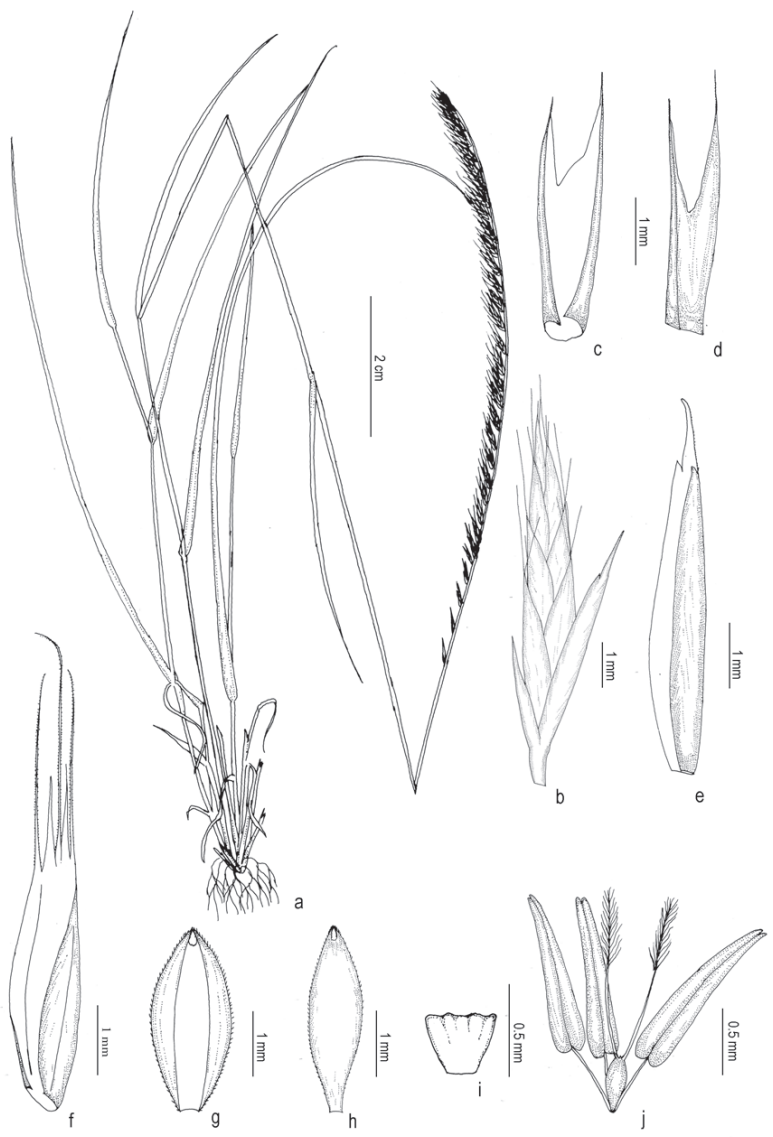

Fig. 21. Tripogon karnatakensis Thoiba \& Pradeep: a. Habit; b. Spikelet; c. \& d. Lower glume; e. Upper glume; f. Lemma; g. Palea - ventral view; h. Palea - dorsal view; i. Lodicule; j. Stamen \& pistil.

Caespitose perennials. Culms 40-60 cm tall, erect, stout, unbranched; nodes glabrous, slightly geniculate. Leaf sheaths 3-8 cm long, closely involute to the culm, persistent, fibrous; ligules narrow membrane, fringed with long hairs at apex, hairs 3-7 mm long; leaf blades $12-23 \times 0.4-0.5 \mathrm{~cm}$, flat to convolute, apex acute-acuminate, scabrid, sparsely villous adaxially and glabrous abaxially, margins entire. Racemes 15-28 × 0.5-0.7 cm, 5060 spikelets loosely or tightly appressed to concavities in rachis; rachis $3-5 \mathrm{~mm}$ long, stout, tri-angular, minutely scabridulose; peduncle 5-10 cm long, stout, glabrous. Spikelets 10-30 × 3-4 mm, linear-lanceolate, leaden green, 7-10-flowered; callus bearded, hairs 1-1.5 mm long; rachilla persistent, 1-1.2 mm long, glabrous, slightly zigzag; lower glumes 5-7 × 1 mm, oblong-lanceolate, asymmetrical, 2-nerved, 2-keeled, bi-lobed, 


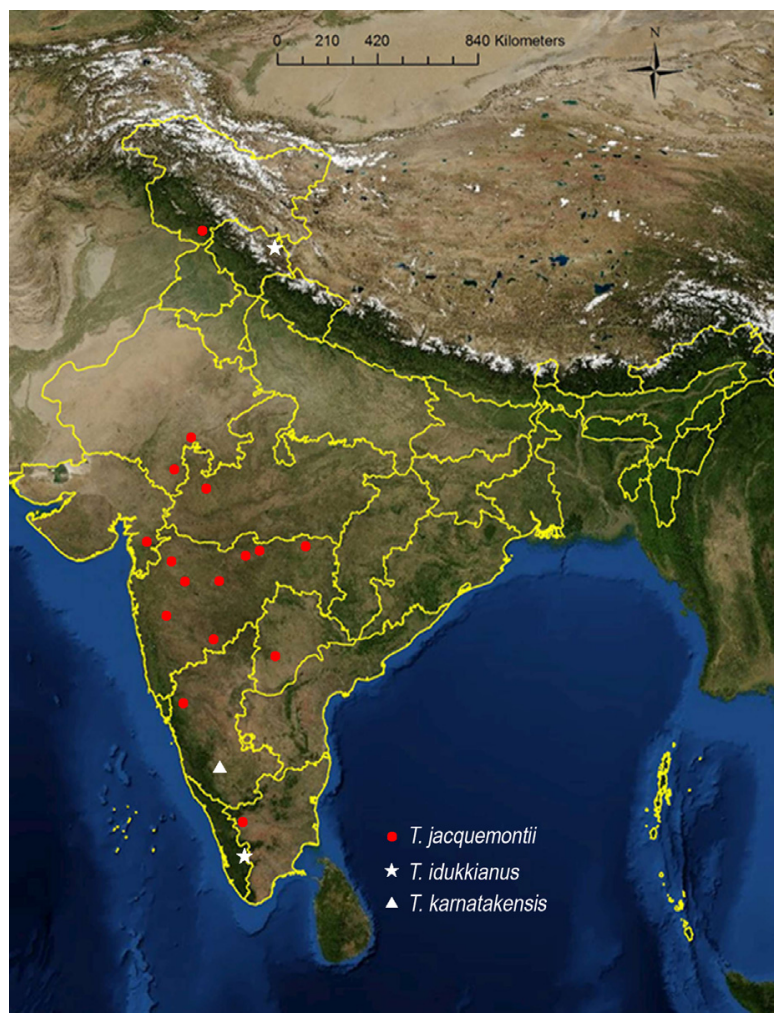

Fig. 22. Distribution of Tripogon jacquemontii Stapf, T. idukkianus Sunil \& Pradeep and T. karnatakensis Thoiba \& Pradeep in India [drawn using ESRI (2011) ArcGIS Desktop: Release 10].

chartaceous, shortly awned at the apex, awns $1 \mathrm{~mm}$ long; upper glumes 6-8 × 0.5-1 mm, symmetrical, elliptic-lanceolate, 1-nerved, 1-keeled, prominentely, scabrid, apex 2-fid, shortly awned from the notch, awns 2.5-3 mm long, scabrid; lemmas 3-3.5 × 1.5-2 mm (excluding awns), narrowly elliptic to ovatelanceolate, 4-lobed, 3-nerved, 3-awned, lobes acuteacuminate, 1-1.5 mm long, median awn 3.7-4.2 mm long, scabrid, straight or curved, lateral awns 2.5-3 mm long; paleas 2.8-3 × 1.7-2 mm, hyaline, ellipticlanceolate, keeled and slightly winged, keels minutely ciliolate along margins, sub-acute to deeply notched at apex; lodicules 2, c. $0.5 \mathrm{~mm}$ long, wedge-shaped, apex coarsely 3-toothed; stamens 3, anthers $1.5-2 \mathrm{~mm}$ long, oblong, filaments $0.5-0.75 \mathrm{~mm}$ long, slender, glabrous; ovary $0.25-0.5 \mathrm{~mm}$ long, obovate, style 2, $0.75-1 \mathrm{~mm}$ long, slender, hyaline, stigma $0.75-1 \mathrm{~mm}$ long, plumose. Caryopses not seen.

Flowering \& fruiting: Flowering and fruiting from September to December.
Habitat: It grows on hill tracts among boulders and also along the Ghat road sides at elevations between 1000-1950 m, in association with Eriocaulon spp. (Eriocaulaceae), Impatiens spp. (Balsaminaceae), Indopoa paupercula, Tripogon bromoides, T. filiformis (all Poaceae) Cyanotis papilionacea and Murdannia dimorpha G.Brückn. (both Commelinaceae).

Distribution: Endemic to South India, hitherto known only from the type locality and adjoining areas.

Specimens examined: INDIA, Karnataka, Chikmagalur district, Baba Budan hills, $1560 \mathrm{~m}$, 13.09.2014, K. Thoiba \& Mohammed Yoonus 137501; Ibid., 13.09.2014, K. Thoiba \& Mohammed Yoonus 137530 (CALI).

Notes: Tripogon karnatakensis resembles T. fliformis, but differs in having flat to convolute, sparsely villous and adaxially scabrid leaves, 15-28 cm long stout leaden green racemes, 2-keeled lower glumes and ciliate paleas with a deep apical notch.

Tripogon lisboae Stapf, Kew Bull. 1892: 84. 1892; Hook.f., Fl. Brit. India 7(22): 286.1896; Bor, Grass. Burma Ceylon India \& Pakistan 522. 1960; S.Moulik, Grass. Bam. India 2: 622. 1997; Kabeer \& V.J.Nair, Fl. Tamil Nadu Grass. 180. 2009; Potdar, Salunkhe \& S.R.Yadav, Grass. Maharashtra 563. 2012. Lectotype (designated by Thoiba \& Pradeep, 2018): INDIA, Rajasthan, Rajputana, Mt. Abu, on rocks, $3500 \mathrm{ft}, 12.10 .1987$, J.F. Duthie 6788 (K [K000245015 digital image!]).

Figs. $23,24 \& 27$

Tufted perennials. Culms 10-120 cm high, terete, erect; nodes glabrous. Leaf sheaths 6-12 cm long, terete, glabrous; ligules absent; leaf blades 20-60 × 0.4-0.7 cm, linear, lanceolate, glaucous, flat or sometimes rolled, sparsely villous adaxially, scabridulous on veins, acuminate at apex. Racemes 10-30 cm long, stout, erect or curved, 15-35 spikelets appressed to concavities in rachis; rachis stout, glabrous or scabrid, 3-12 mm long; peduncles 8-15 cm long, glabrous. Spikelets 5-13 × 1-2 mm narrowly elliptic, linear, distant, olive green, 5-10flowered; callus bearded, hairs c. $0.3 \mathrm{~mm}$ long; rachilla $0.1-0.2 \mathrm{~mm}$ long, glabrous, straight or zig 


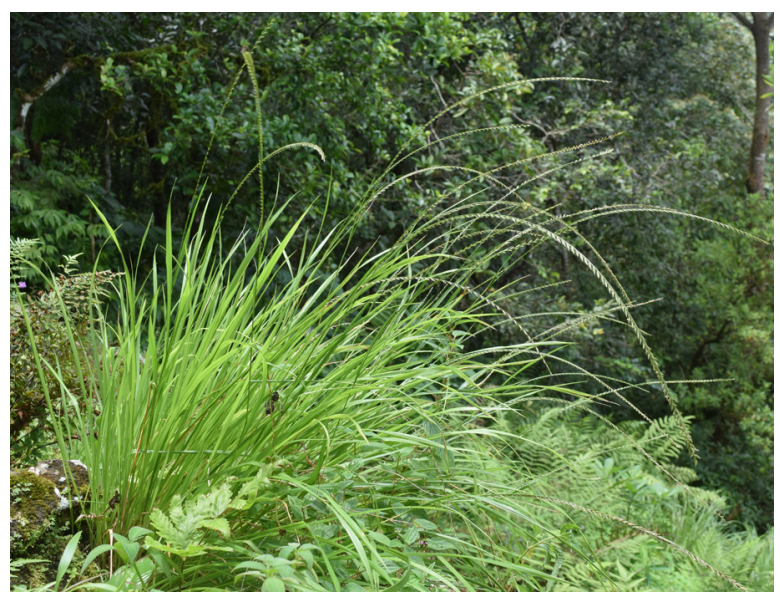

Fig. 23. Tripogon lisboae Stapf in its natural habitat (Photo by A.K. Pradeep)

zag, not persistent; lower glumes 2.5-4 × 0.5-0.8 $\mathrm{mm}$, notched on one-side, ovate-lanceolate, $1-$ nerved, 1-keeled, glabrous, and acute at apex; upper glumes 3-5.3 × 0.5-0.8 mm, elliptic-ovate, 1keeled, 1-3-nerved, lateral nerves not prominent, apex 2-toothed, shortly aristate; lemmas 2.8-3.2 $\times$ 0.6-0.8 mm, narrowly ovate, 2-lobed, 3-nerved, slightly keeled, 1-awned, awns 1-2.2 mm long, scabridulous, lateral lobes membranous; paleas $2.8-3.2 \times 0.5-1 \mathrm{~mm}$, elliptic-lanceolate, narrowly winged, hyaline, 2-keeled, keels ciliate, acute at apex; lodicules 2, c. $0.3 \mathrm{~mm}$ long, slightly cordate, 3-toothed; stamens 3, anthers 1-1.8 mm long, oblong, filaments $0.5-0.75 \mathrm{~mm}$ long, slender, glabrous; ovary $0.3-0.5 \mathrm{~mm}$ long, obovate, styles 2, 0.5-0.75 mm long, slender, hyaline, stigma 0.75$1.2 \mathrm{~mm}$ long, plumose, creamy white. Caryopses 1.4-2.2 $\times$ c. $0.5 \mathrm{~mm}$, narrowly oblong, terete, light brown.

Flowering $\&$ fruiting: Flowering and fruiting from September to November.

Habitat: This species is found growing on the cliffs of moist rocks along Ghat road sides and also grasslands at elevations of 600-1800 m. Occasionally seen in association with Begonia malabarica Lam. (Begoniaceae), Chlorophytum heynei Baker (Asparagaceae), Chrysopogon hackelii (Hook.f.) C.E.C.Fisch., Eragrostis collinensis Vivek, G.V.S.Murthy \& V.J.Nair, Tripogon bromoides (all Poaceae) and Impatiens sp. (Balsaminaceae).
Distribution: India, fairly common in southwest regions of the Peninsula.

Specimens examined: INDIA, Goa, North Goa district, Dicholi, 12.09.1970, R.K. Kochhar 154177; Naruja, tableland Keyna, 29.11.1978, R.K. Kochhar 157977 (BSI); Surla, Satari, 02.10.2005, Harshala Gad \& M.K. Janarthanam 244 (Goa University Herbarium); South Goa district, s.loc., 11.09.2016, Syam Radh S. \& K. Thoiba 146739 (CALI). Karnataka, Chikmagalur district, Kudremukh National Park, 18.09.2015, K. Thoiba \& A.K. Pradeep 144145 (CALI); Shimoga district, Mookambika Wildlife Sanctuary, Khodachadri, 09.09.2015, K. Thoiba 144163 (CALI). Kerala, Idukki district, way to Vagamon, 10.10.2015, K. Thoiba \& C. Pramod 146606 (CALI); Wayanad district, Periya, Gurukula Botanical Sanctuary, 22.08.2015, K. Thoiba \& A.K. Pradeep 144144 (CALI). Madhya Pradesh, Hoshangabad district, Pachmaxi road, Bori R.F., 06.10.1960, J. Joseph 11254 (MH). Maharashtra, Kolhapur district, Amba Ghat, 06.11.2016, K. Thoiba 146733 (CALI); Tillari, 16.09.2001, s.coll. s.n. (SUK); Mumbai district, Mumbai hills, Thanae range, 09.09.1968, K.V. Billore 116607 (CAL); Pune district, Bhimashankar, 30.03.1957, G.S. Puri 12598 (BSI); Ibid., 09.10.1962, K.P. Janardhanan 81740 (CAL); Junnar, Aie, 08.2007, S.B. Nagarkar 11526 (AHMA); Khandala, 20.09.1902, G.A. Gammie 15442; Meroli, 15.09.1957, S.D. Mahajan 17184 (BSI); Kukdeshwar, 07.10.2001, S.B. Nagarkar s.n. (AHMA); Lonavla, Ravine above Bashi lake, 28.09.1964, B. Venkata Reddi 98796 (BSI); Sinhagad, 05.09.2010, s.coll. 3422 (CALI); Rairashwar, 17.09.1958, V.D. Vartak 13860 (CAL); Vazirgarh fort, 26.12.1944, H. Santapau 5648; Ibid., 29.12.1945, H. Santapau 8335 (BLAT); Raigad district, Karnala, 29.09 1976, V.D. Vartak 1126 (AHMA); Matheran, Hart point, 08.10.2003, R.K. Singh 188778 (BSI); Satara district, Carnac point, 12.10.1960, M.Y. Ansari 67659; on the top of the fort, Muhshi, Ambawane, 06.09.1964, B. Venkata Reddi 99051; Khandala, 09.1907, A.Meebold 9121; Mahablaeshwar, Koyna, Tadorhi, 24.11.1978, R.K. Kochhar 157834; Ibid., 05.10.1957, G.S. Puri 25639 (CAL); Panchgani, 10.1908, E. Blatter 338; 


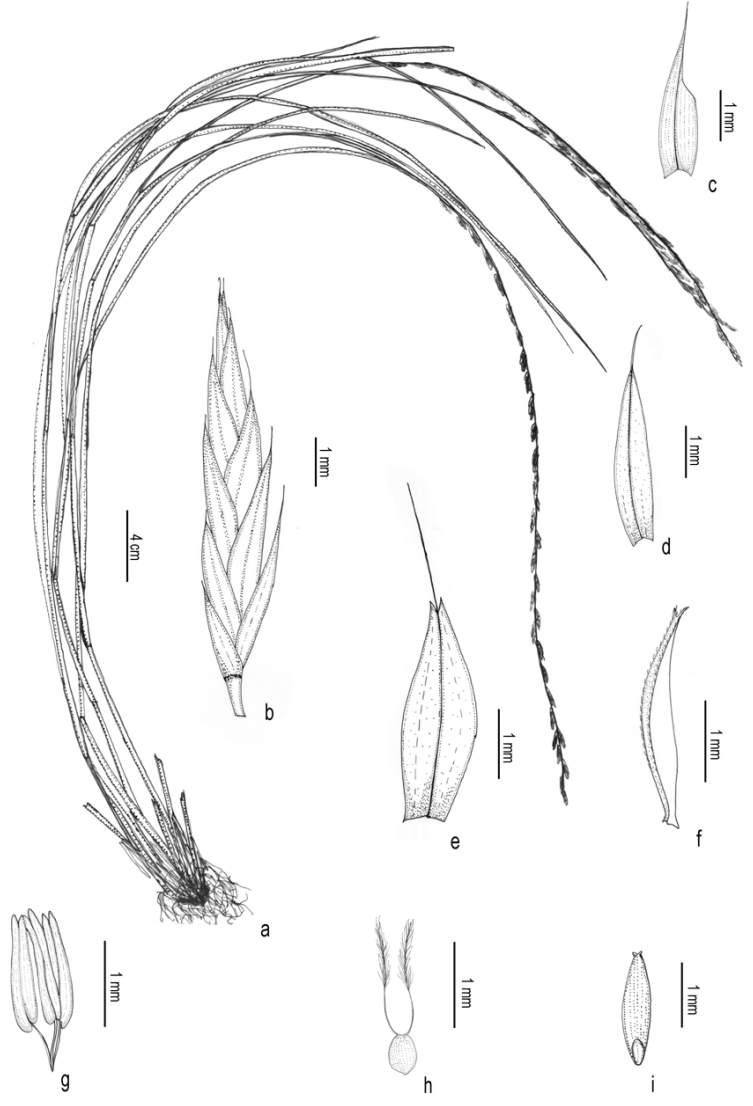

Fig. 24. Tripogon lisboae Stapf: a. Habit; b. Spikelet; c. Lower glume; d. Upper glume; e. Lemma; f. Palea; g. Stamen h. Pistil; i. Caryopsis.

Mahabalaeshwar, 14.09.1958, H. Santapau 22737 (BLAT); Sindhudurg district, Amboli Ghat, Nanapuri, 31.10.1969, B.G. Kulkerni 119234; Mahadeogad, 09.10.1970, B.G. Kulkerni 121545 (BSI); s.loc., 30.09.2001, s.coll., s.n.; s.loc., s.die., M.M. Sardesai s.n. (SUK); Sangli district, Dongarwadi, 26.01.2005, Aparna Watve 00511 (AHMA). Rajasthan, Banswara district, s.loc., 20.03.1976, V. Singh 2913 (CAL); Churu district, Ratangarh, 04.10.1970, B.M. Wadhwa 128120 (BSI); Jaipur district, Devaliya, 29.09.1960, R.S. Rao 6606 (BSI); Jhalawar district, s.loc., 17.09.1964, B.M. Wadhwa 5405 (CAL); Jhunjhunu district, Khola hill, Sirohi, 24.10.1960, P.C. Nanda 2086 (CAL); Jodpur district, Mahalkari, Manar, 17.10.1906, s.coll. 2 (BSI); Sirohi district, Mount Abu, Guru Shikhar road, 10.11.1959, S.K. Jain 660177; on way to Devaliya, 29.09.1960, R.S. Rao 66643 (BSI). Tamil Nadu, Coimbatore district, Lambden's peak, 03.06.1954, D. Daniel Sundararaj s.n. (MH); Dindigul district,
Kodaikanal, Law's Ghat road, 08.09.1985, K.M. Matthew 42249 (RHT).

Notes: It resembles T. malabaricus, but differs in having flat or rolled leaves, inconspicuous ligules, racemes being straight or curved, upper glumes shortly aristate and awns as long as or shorter than the lemmas.

Tripogon longearistatus Hack. ex Honda, Bot. Mag. Tokyo 41: 11. 1927, as “longe-aristatus"; Lee, Manual Korean Grass. 244. 1966; Koyama, Grass. Japan \& Neighbour Regions 271: 1987; Osada, Illustr. Grass. Japan 1: 466. 1989; S.L.Chen, Fl. Reipubl. Popul. Sin. 10(1): 61. 1990; S.M.Philips \& S.L.Chen, Kew Bull. 57: 920. 2002; Z.Y.Wu, P.H.Raven \& D.Y.Hong in S.L.Chen \& S.M.Philips, Fl. China 22: 468. 2006. Tripogon chinensis (Franch.) Hack. var. longearistatus Hack. ex Honda, Bot. Mag. (Tokyo) 41: 11. 1927. Lectotype (designated here): KOREA, Cheju-do, Quelpaert, 1908, Taquet 3425 (TI [TI00016318 digital image!]).

Tripogon longearistatus Nakai, Veg. Isl. Quelpaert: 19: 147. 1914, nom. nud.

Tripogon coreensis (Hack.) Ohwi var. longearistatus Hack. ex T. Mori, nom. nud.

Tripogon longearistatus Honda var. japonicas Honda, Bot. Mag., Tokyo 41: 12. 1927. Tripogon japonicus (Honda) Ohwi, Acta Phytotax. Geobot. 4: 63. 1935; Tripogon longearistatus Honda subsp. japonicus (Honda) T.Koyama, Grass. Japan \& Neighbour. Regions 271: 532. f.103. 1987. Type: JAPAN, Hondo, s.d., Ando 69 (holo TI!).

Tripogon panxianensis H.Peng, Acta Bot. Yunnan. 13 (2): 147. 1991. Type: CHINA, Guizhou, Panxian, 28.09.1988. Gesuo, C.Z. Dang 1010 (holo HGAS; iso YUNU).

Figs. 25-27

Caespitose perennials. Culms 15-30 cm high, erect; nodes glabrous. Leaf sheaths $4-10 \mathrm{~cm}$ long, papery, ribbed, glabrous; ligules ciliolate membranous; leaf blades $4-17 \times$ c. $0.1 \mathrm{~cm}$, linear, convolute, glabrous or loosely pilose adaxially and glabrous abaxially, acuminate or attenuate at apex. Racemes $8-30 \mathrm{~cm}$ 
long, slightly flexuous, spikelets loosely arranged in rachis; rachis slender; peduncles $5-8 \mathrm{~cm}$ long, glabrous. Spikelets 4.5-10.5 × 1-1.3 mm, linearlanceolate, pale green to dark grey; 4-7(-12)flowered; callus bearded, hairs $0.5-1 \mathrm{~mm}$ long; rachilla $0.4-0.5 \mathrm{~mm}$ long, glabrous, straight or zig zag, not persistent; lower glumes 2.5-3 × 0.5-0.7 $\mathrm{mm}$, linear-lanceolate, asymmetrical, broadened or toothed on one side, 1-nerved, 1-keeled; keels slightly scabrid, sub-acute or acuminate at apex; upper glumes 3.5-4.5 × 0.5-0.7 mm, lanceolateoblong, 1-nerved, 1-keeled, acuminate-rostrate or emarginated and mucronate at apex; lemmas 2.3$3.3 \times 0.5-0.8 \mathrm{~mm}$ (excluding awns), ellipticlanceolate, 3-nerved, bidentate, median awn 3.6-8 $\mathrm{mm}$ long, stiff, scabrid, strongly reflexed, teeth acute; lateral veins extended into $0.2-0.4 \mathrm{~mm}$ long awns arising free from lemma tooth or from its outer margins, lateral awn size may be equal or not; paleas 2-2.5 × 0.4-0.6 mm, lanceolate, hyaline, 2-keeled, narrowly winged, keels ciliolate, apex acute or bilobed; lodicules 2, 0.2-0.3 mm long, dentate at apex; Stamen 1, anthers 1-1.5 mm long; ovary 0.2-0.3 $\mathrm{mm}$ long, styles $2,0.2-0.3 \mathrm{~mm}$ long, slender, hyaline, stigma $0.4-0.5 \mathrm{~mm}$, feathery. Caryopses 1-1.6 × 0.1-0.2 mm, linear-lanceolate, cylindric, light brown.

Flowering and fruiting: Flowering and fruiting from September to December.

Habitat: On rocky slopes and also in river beds between 300-1500 m altitude.

Distribution: China, India, Japan and Korea.

Specimens examined: INDIA, Meghalaya, East Khasi hills district, Mawlynnong, 1496 m, 11.10.2017, Santhosh Nampy \& Vishnu Mohan 156870a, 156870b, 156870c, 156870d, 156870e (CALI). Sikkim, West Sikkim district, Tashiding, on the way to Phamerong waterfalls, 29.07.2018, Nikhil Krishna \& K. Thoiba 159815 (CALI).

Notes: Honda (1927) described T. longearistatus based on specimens collected from Quelpeart (Japan, Korea). He cited six specimens "Corea: ins. Quelpaert (Taquet 1882, Anno 1908); ibidem

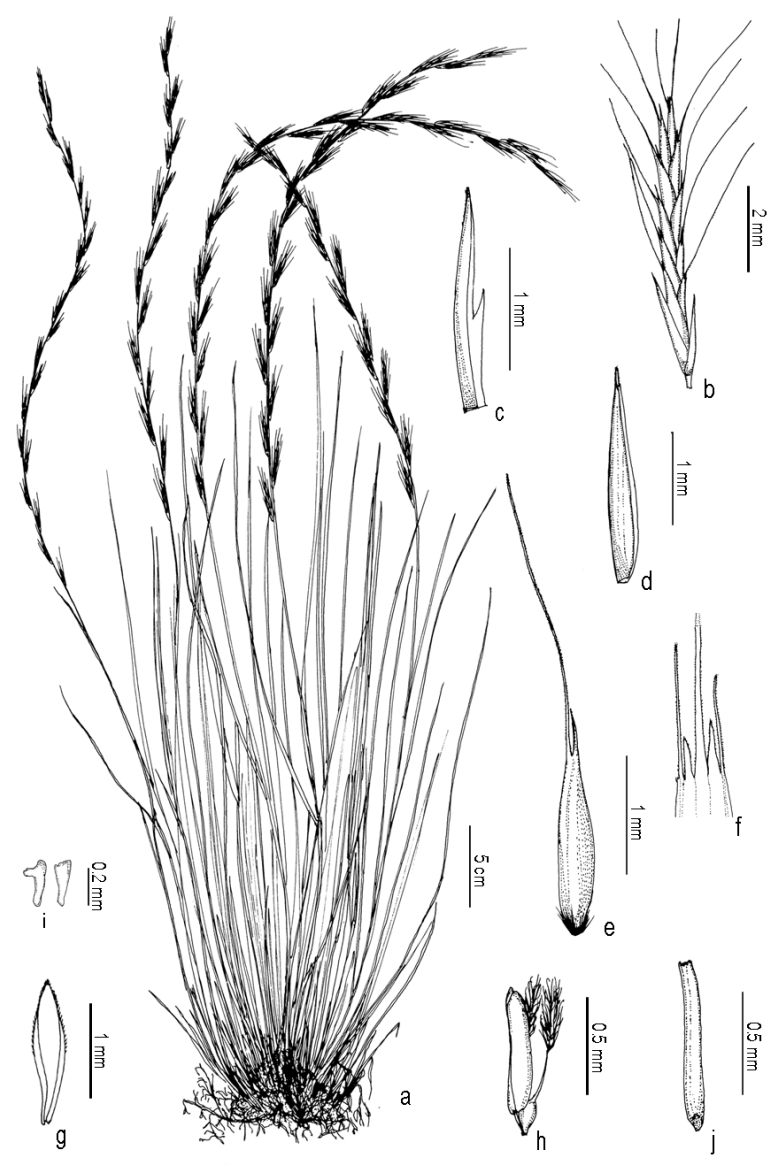

Fig. 25. Tripogon longearistatus Hack. ex Honda: a. Habit; b. Spikelet; c. Lower glume; d. Upper glume; e. Lemma; f. Enlarged portion of lemma apex; g. Palea; h. Stamen \& pistil; i. Lodicule; j. Caryopsis.

(Taquet 3425, Anno 1909); ibidem (T. Nakai 4845, Anno 1917). Planta endemica” without designating a type. According to Phillips and Chen (2002), among the materials cited, three syntypes are available at the Herbarium of the University of Tokyo (TI). While searching for the original specimens at TI, we could trace only two relevant sheets. Among them, the sheet Taquet 3425 (TI [TI00016318 digital image!]) bears the type label, and also a determination slip affixed by M. Honda in the same year. The other sheet Nakai 4845 (TI [TI100016317 digital image!]) was also determined by Honda in 1925 but with three specimens mounted on a single sheet without any type labels. Therefore, the sheet Taquet 3425 (TI [TI00016318 digital image!]) carrying a well preserved specimen matching exactly with the protologue is designated here as the lectotype of T. longearistatus. 


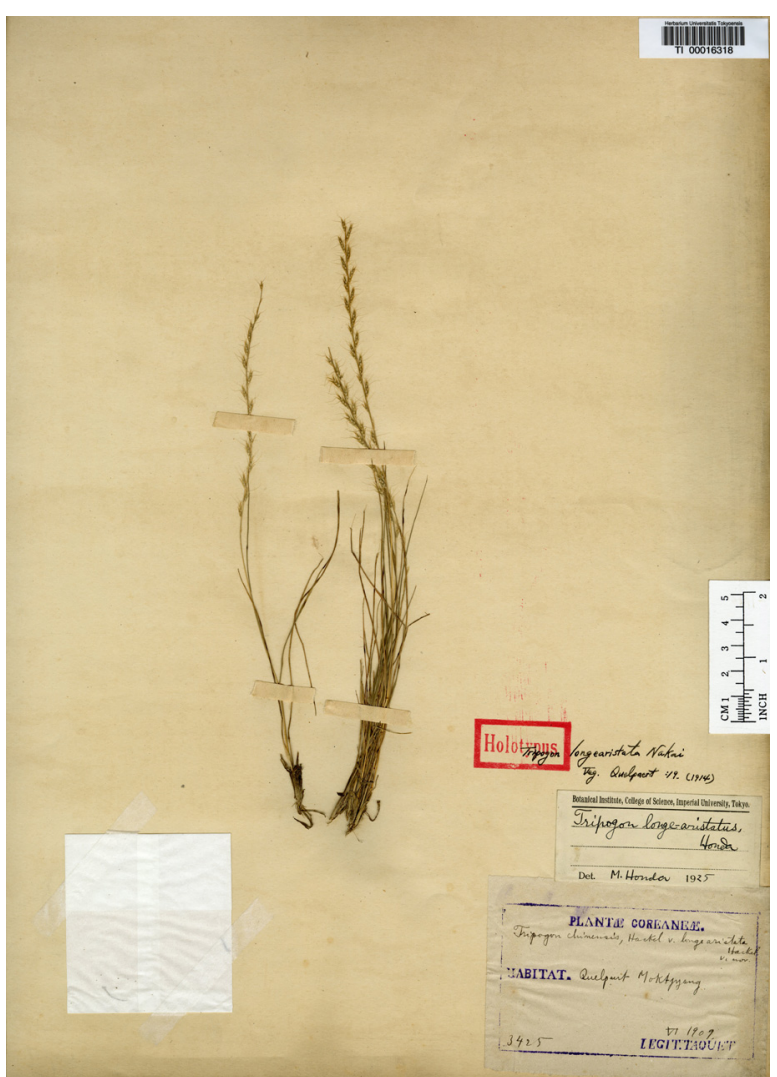

Fig. 26. Lectotype of Tripogon Iongearistatus Hack. ex Honda (TI 00016318) designated here. (C) The Board Trustees for the University of Tokyo, Japan. Reproduced with permission.

Tripogon longearistatus closely resembles T. filiformis Nees ex Steud., but can easily be distinguished by its widely spaced spikelets with stiff strongly reflexed awns, lateral awns arising free from lemma tooth or from its outer margins, and by its solitary anther. However, specimens of $T$. filiformis with laxer racemes than usual, or with a tendency for the awns to reflex, and also young specimens of $T$. longearistatus with straight awns, can cause difficulty. There is little overlap in their geographical range. Tripogon filiformis is an upland and high altitude species, whilst T. longearistatus is confined to the eastern lowlands (Phillips \& Chen, 2002; Chen \& Phillips, 2006). Thorough examination of the protologue and type (Royle s.n., (K [K000245038 digital image!]) of T. filiformis showed it as a distinct species. Tripogon filiformis is characterized by its filiform, lax and densely pilose leaves, upper glumes 2-toothed below the apex, lemmas 4-lobed, 3-awned, median awns twice as long as the lemma or more.
Tripogon mahendragiriensis Chorghe, Sang.Dey, K.Prasad, Prasanna \& Y.V.Rao, Nord. J. Bot. 33(6): 655. 2015. Type: INDIA, Odisha, Gajapati district, Mahendragiri hill, N $18^{\circ}$ 58'04.3", E 084 21'57.4', 1478 m, 17.09.2014, Chorghe \& Prasad 4210 (holo BSID!; iso CAL [CAL0000025360!]).

Fig. 27

Caespitose perennials. Culms 15-20 cm high, erect; nodes glabrous. Leaf sheaths $3-5 \mathrm{~cm}$ long, linear, glabrous; ligules ciliate, cilia $0.5 \mathrm{~mm}$ long; leaf blades $4-10 \times 0.05 \mathrm{~cm}$, linear, filiform, sparsely covered with white hairs. Racemes $5-10 \mathrm{~cm}$ long, spiciform, spikelets loosely arranged in rachis; rachis flat, glabrous; peduncle 7-12 cm long. Spikelets 4.5-5.2 × $1 \mathrm{~mm}$, linear-lanceolate, laterally compressed, 4-5-flowered with reduced florets near apex; lower glumes $2-2.25 \times$ c. $0.50 \mathrm{~mm}$, linearlanceolate, shallowly lobed on one-side, 1-nerved, 1-keeled, acuminate at apex; upper glumes 3.5-3.7 × c. $0.5 \mathrm{~mm}$, linear-lanceolate, 1-nerved, 1-keeled,

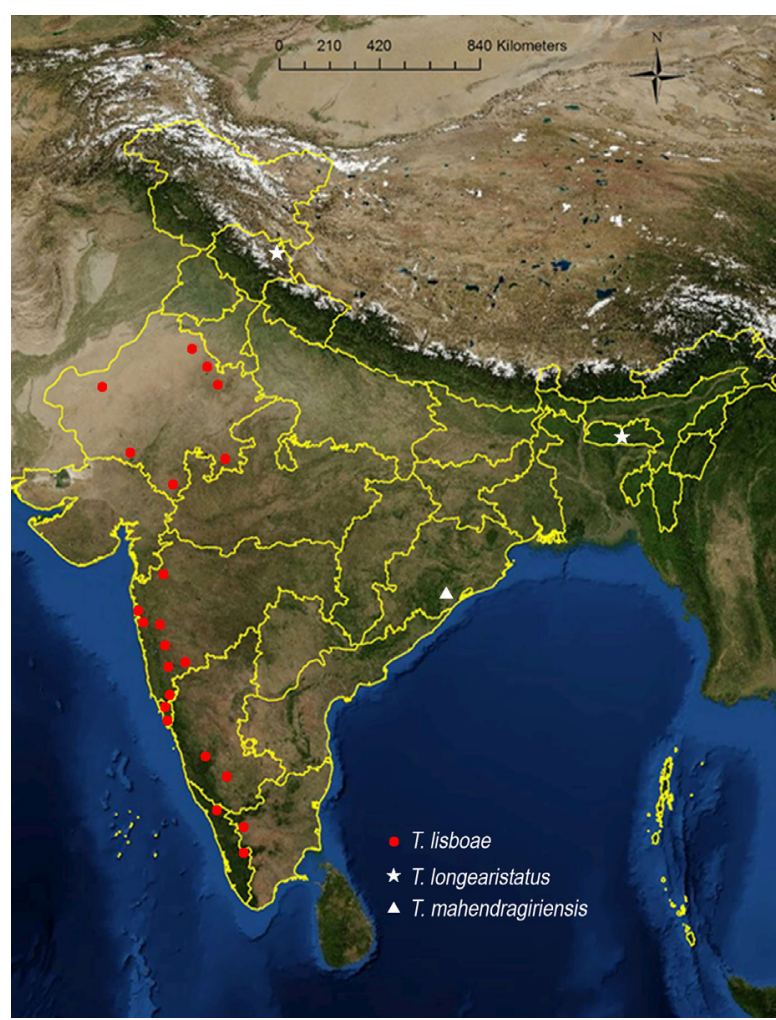

Fig. 27. Distribution of Tripogon lisboae Stapf, T. Iongearistatus Hack. ex Honda and T. mahendragiriensis Chorge, Sang.Dey, K.Prasad, Prasanna \& Y.V. Rao in India [drawn using ESRI (2011) ArcGIS Desktop: Release 10]. 
acuminate at apex; lemmas 2.8-3.1 × 0.7-0.9 mm, lanceolate, bearded at base, 3-nerved, 1-keeled, unlobed, 3-awned, median awn 1.8-2.2 mm long, shorter than lemma, arising from the median nerve, lateral awns $0.5 \mathrm{~mm}$ long; paleas 2.6-2.8 $\times 0.6 \mathrm{~mm}$, oblanceolate, hyaline, 2-keeled, keels ciliate, acute at apex; stamens 2, anthers $1.3 \mathrm{~mm}$ long; ovary 0.4 $\mathrm{mm}$ long, styles 2 , slender, hyaline, stigma feathery. Caryopses 1.5-1.6 × 0.5-0.6 mm, oblanceolate or oblong-elliptic, light brown.

Flowering \& fruiting: Flowering and fruiting in September.

Habitat: It was found growing on rocks above 1400 m elevation associated with Arundinella setosa Trin., Garnotia tenella Janowski and Jansenella griffithiana (Müll.Hal.) Bor (all Poaceae).

Distribution: Endemic to India, hitherto known only from the type locality.

Notes: The authors were not able to collect $T$. mahendragiriensis, and the description provided here is based on the type collection. The species is similar to Tripogon humilis and T. purpurascens, but differs mainly in having longer culms, leaf blades and racemes; lemmas unlobed, 3-awned and two anthers.

Tripogon malabaricus Thoiba \& Pradeep, J. Bot. Res. Inst. Texas 8(2): 523. 2014, as "malabarica". Type: INDIA, Kerala, Kozhikode district, Malabar Wildlife Sanctuary, Kakkayam, 990 m, N 11 33.037', E $075^{\circ} 55.024 '$ ' 19.09.2013, Thoiba Kottekkattu 134436 (holo BRIT!; iso CAL!, CALI [CALI112096!], K!, MH [MH00003235!]).

Figs. $28,29 \& 32$

Tufted perennial herb. Culms 25-65 cm high; nodes glabrous. Leaf sheaths 10-18 cm long, linearlanceolate, coriaceous, villous adaxially, apex pubescent; ligules indistinct, tuft of $2.5-3.5 \mathrm{~mm}$ long hairs at apex; leaf blades 30-70 × 0.2-0.8 cm, linear-lanceolate, flat, margins slightly scabridulous or scabrid, pubescent with short white papillose hairs adaxially and glabrous abaxially. Racemes 15-40 cm long, 30-55 spikelets tightly arranged in rachis; rachis rigid, stout, glabrous; peduncles 8 $15 \mathrm{~cm}$ long, glabrous. Spikelets 13-14 × 1.5-2 mm, linear, distant, dorsiventrally flattened, 4-7flowered; callus bearded; rachilla $1 \mathrm{~mm}$ long, glabrous; lower glumes 2.5-4 × 1-1.5 mm, coriaceous, ovate-lanceolate, 1-nerved, 1-keeled, keels slightly scabrid, acuminate at apex; upper glumes 4.5-6 × 1-1.5 mm, lanceolate, coriaceous, 1-3-nerved, 1-keeled, scabrid, acute at apex; lemmas 3-5 × 1.5-2 mm, ovate-lanceolate, 3-nerved, slightly keeled, scabrid, 1-awned, awns 3-5 mm long, scabrid, sometimes slightly geniculate towards apex; paleas 3-5 × 1-1.5 mm, obovate, hyaline, 2-keeled, ciliate, subulate at apex; lodicules 2, 0.25-0.5 mm long, truncate at apex, coarsely 3-toothed; stamens 3, anthers 1.5-2 mm long, oblong, filaments $1 \mathrm{~mm}$ long, slender, glabrous; ovary $0.5-0.75 \times 0.25-0.5 \mathrm{~mm}$, obovate, styles 2, 0.5-0.75 mm long, slender, hyaline, stigma $0.75 \mathrm{~mm}$ long, plumose, creamy white. Caryopses not seen.

Flowering \& fruiting: Flowering and fruiting from late July to early November.

Habitat: This species grows from 950-1500 m altitude on steep granitic cliffs, road cuts and wet rocky hillsides in association with Chlorophytum malabaricum Baker (Asparagaceae), Impatiens scapiflora B.Heyne, I. gardneriana Wight (Balsaminaceae), Kleinia grandiflora (DC.) Rani (Asteraceae), Pouzolzia auriculata Wight, P. bennetiana Wight (both Urticaceae), Themeda triandra Forssk. and Panicum spp. (both Poaceae).

Distribution: Endemic to South India, hitherto known only from the type locality and adjoining areas.

Specimens examined: INDIA, Kerala, Kozhikode district, Malabar Wildlife Sanctuary, Kakkayam, 09.11.2014, K. Thoiba 137545; Vayalada, Thalayad, Kakkayam, 16.09.2017, Nikhil Krishna \& K. Thoiba 146746 (CALI); Malappuram district, Thamburankolli, Koodaranji, 30.10.2013, K. Thoiba 134480 (CALI).

Notes: Dey and Prasanna (2019) reduced this taxon to synonymy under T. major subsp. vellarianus (Pradeep) Sang.Dey \& Prasanna. This conclusion 


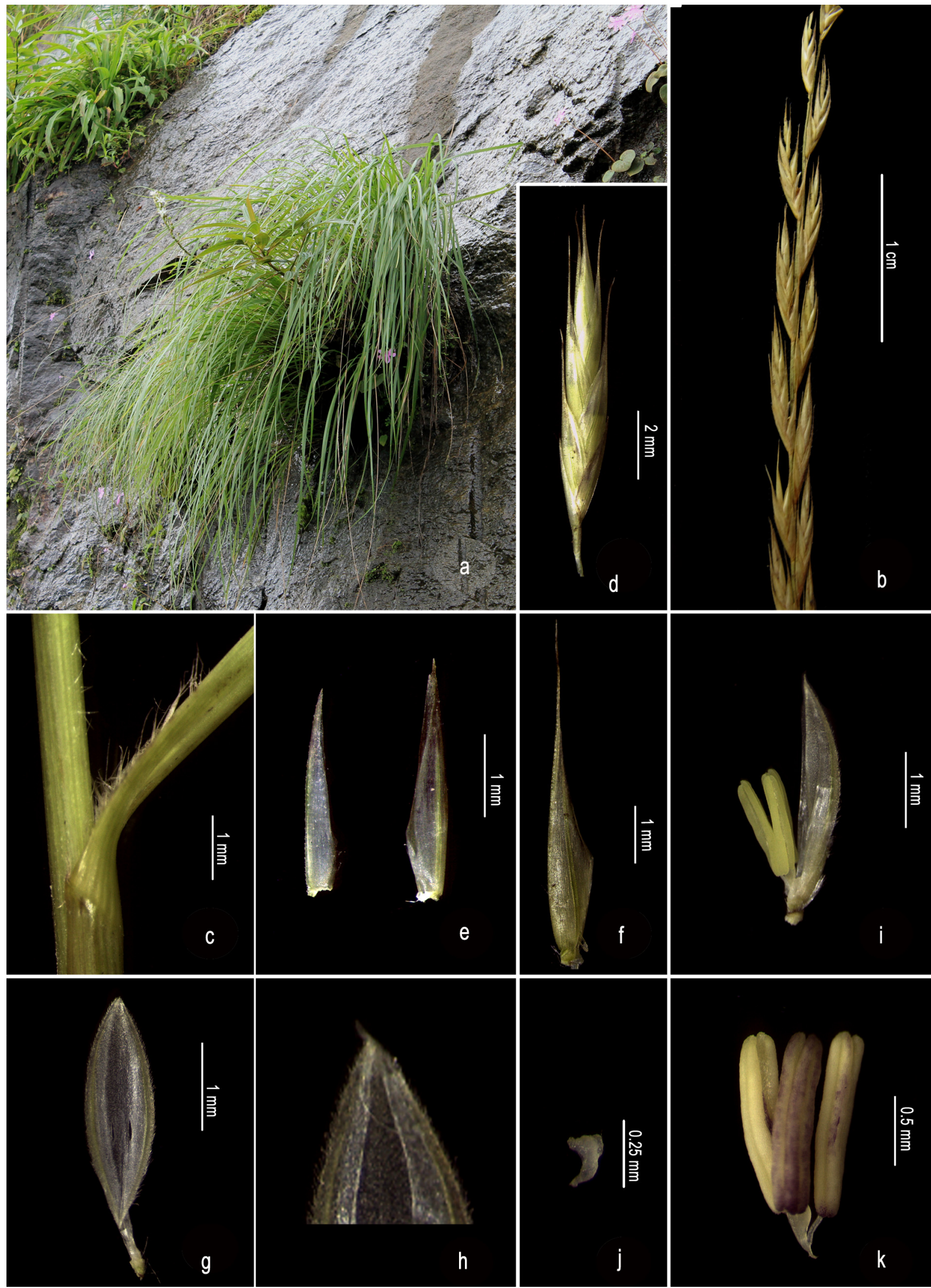

Fig. 28. Tripogon malabaricus Thoiba \& Pradeep: a. Habit; b. Enlarged portion of raceme; c. Ligule; d. Spikelet; e. Glumes; f. Lemma; g. Palea; h. Enlarged portion of palea apex; i. Palea with flower; j. Lodicules; k. Stamen \& pistil. 


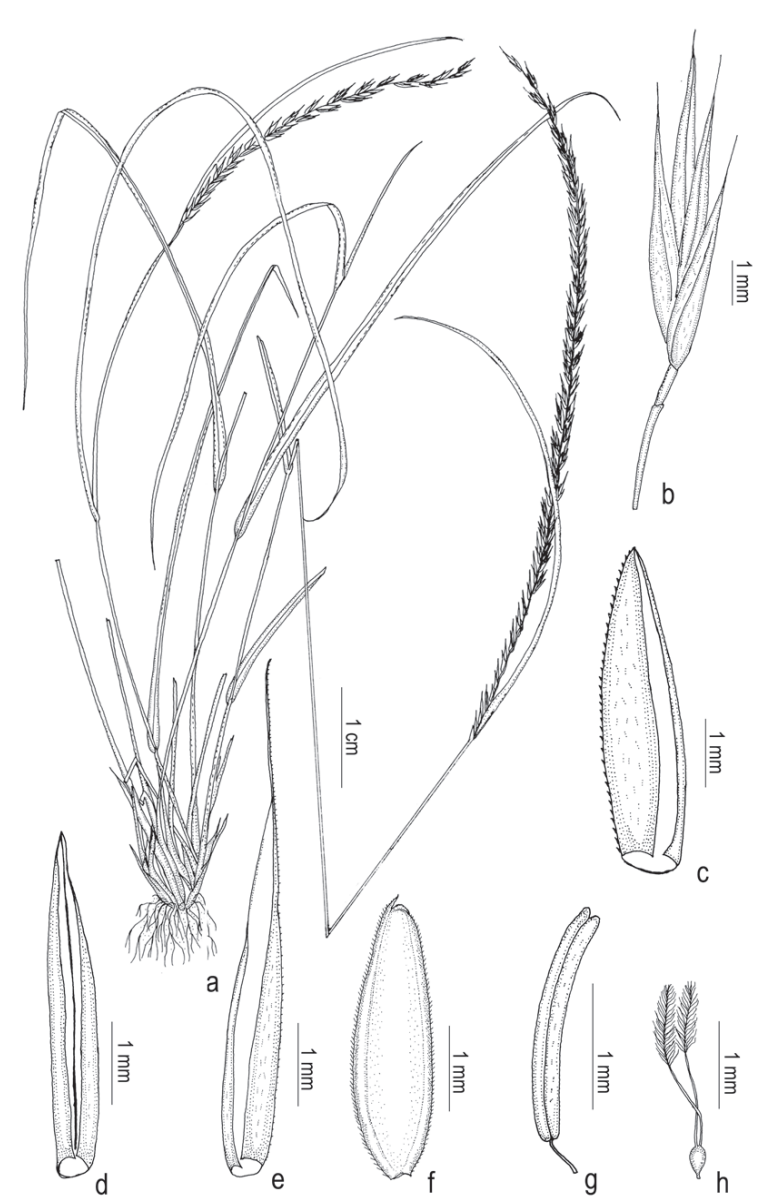

Fig. 29. Tripogon malabaricus Thoiba \& Pradeep: a. Habit; b. Spikelet; c. Lower glume; d. Upper glume; e. Lemma; f. Palea; g. Stamen; h. Pistil.

is based on a comparison of single specimen each of both the taxa available at CAL. Our field observations of both taxa do not support this finding. Tripogon malabaricus differs from $T$. vellarianus and T. lisboae in having linear, coriaceous, villous leaves, 13-14 mm long spikelets with 4-7florets, lemma with straight or geniculate, scabrid awns and keeled palea with a subulate apex.

Tripogon munnarensis Thoiba \& Manudev, Phytotaxa 272(2): 130. 2016. Type: INDIA, Kerala, Idukki district, Munnar, Udumbanchola, near Mundiyaruma, 1430 m, 16.12.2014, T. Kottekkattu \& A.K. Pradeep 144105 (holo CAL!; iso CALI [CALI112103!], MH!, K!).

Figs. 30 \& 32

Caespitose perennials. Culms 5-15 cm high erect, unbranched; nodes glabrous, slightly geniculate.
Leaf sheaths $2-5.5 \mathrm{~cm}$ long, closely involute to the culm, persistent, fibrous; ligules obscure, fringed with long hairs at apex, hairs 1-2 mm long; leaf blades $5-15 \times 0.18-0.25 \mathrm{~mm}$, lanceolate, flat to convolute, densely villous adaxially and sparsely villous abaxially, margins entire, minutely pungent at apex. Racemes 7-15 cm, contracted, 14-35 spikelets loosely or tightly arranged in rachis; rachis 0.5-1 cm long, stout, quadrate, glabrous; peduncles 5-10 cm long, stout, glabrous. Spikelets 10-16 × 1-2.5 mm, linear-lanceolate, 10-16-flowered, younger florets with pinkish dots; callus bearded, barbate in front at the base of rachilla internode, hairs 1-1.5 mm long; rachilla not persistent, 1-1.2 $\mathrm{mm}$ long, glabrous, slightly zig-zag; lower glumes 1-2.7 ×0.5-1 mm, oblong-lanceolate, asymmetrical, chartaceous, 1-nerved, acute at apex; upper glumes 3-4.5 × 0.5-1 mm, symmetrical, elliptic-lanceolate, 1-nerved, 1-keeled, prominently scabrid, 2-fid at apex, shortly awned from the notch, awns 0.3-0.5 mm long, scabrid; lemmas 3-3.5 × 1-1.5 mm (including awn), narrowly elliptic to ovatelanceolate, 4-lobed, 3-nerved, 3-awned, lobes acute-acuminate, $0.3-0.5 \mathrm{~mm}$ long, aristate, median awn 1-1.5 mm long, scabrid, almost straight or curved, lateral awns $0.5-0.8 \mathrm{~mm}$ long; paleas $1.5-$ $3 \times 0.6-2 \mathrm{~mm}$, hyaline, elliptic-lanceolate, keeled and slightly winged, keels minutely ciliolate along the margins distally, sub-acute to deeply notched at apex; lodicules 2, 0.5-0.8 mm long, wedgeshaped, apex coarsely 3-toothed; stamens 3, anthers 1-1.5 mm long, oblong, filaments $0.4-0.5 \mathrm{~mm}$ long, slender, glabrous; ovary $0.3-0.5 \mathrm{~mm}$ long, obovate, style 2, 0.5-0.75 mm long, slender, hyaline, stigma $0.5-0.75 \mathrm{~mm}$ long, plumose. Caryopses not seen.

Flowering \& fruiting: Flowering and fruiting from September to early December.

Habitat: It grows on hill sides among boulders and also along the Ghat road sides at elevations between 1000-1500 m, in association with Chrysopogon nodulibarbis, Tripogon bromoides, T. sivarajanii (all Poaceae), Henckelia incana (Gesneriaceae), Eriocaulon spp. (Eriocaulaceae) and moss species. 
Distribution: Endemic to India, hitherto known only from the type locality and adjoining areas.

Specimens examined: INDIA, Kerala, Idukki district, Kambakal Medu, Munnar, 26.09.2014, K.M. Manudev 138906 (CALI); Munnar, Udumbanchola, near Mundiyaruma, 16.12.2014, K. Thoiba, A.K. Pradeep \& T. Mohammed Yoonus 144105 (CAL, CALI, K, MH); Ramakkalmedu, 23.10.2017, A.K. Pradeep \& K. Thoiba 146752 (CALI).

Notes: Tripogon munnarensis is similar to $T$. narayanae, but differs from it in having 10-16 $\mathrm{mm}$ long spikelets with 10-16 florets, rachilla $0.5-0.8$ $\mathrm{mm}$ long, apically acute asymmetrical lower

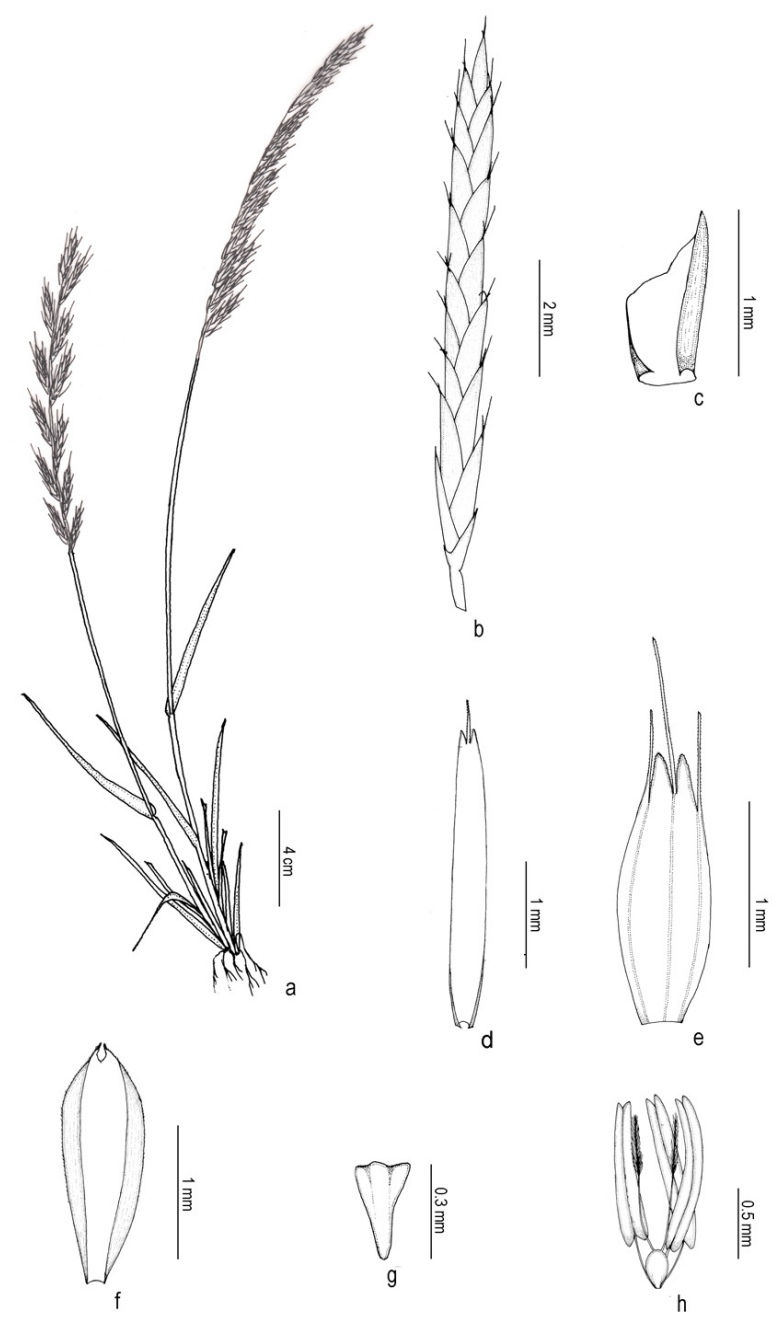

Fig. 30. Tripogon munnarensis Thoiba \& Manudev: a. Habit; b. Spikelet; c. Lower glume; d. Upper glume; e. Lemma; f. Palea; g. Lodicule; h. Stamen \& pistil. glumes, median awns 1-1.5 mm long, almost straight or curved, and lateral awns $0.5-0.8 \mathrm{~mm}$ long.

Tripogon narayanae Sreek., V.J.Nair \& N.C.Nair, J. Bombay Nat. Hist. Soc. 80(1): 196. 1983, as “narayanii"; Sreek. \& V.J.Nair, Fl. Kerala Grass: 401, t. 84. 1991; S.Moulik, Grass. Bamb. India 2: 622. 1997; Kabeer \& V.J.Nair, Fl. Tamil Nadu Grass: 181. 2009; Sasidh., Biodiv. Doc. Kerala-Fl. Pl. 595. 2004. Type: INDIA, Kerala, Idukki district, Eravikulam National Park, 2000 m, 28.08.1980, P.V. Sreekumar 68412 (holo (CAL [CAL0000002441!]; iso K [K000245043 digital image!], $\mathrm{MH}$ [MH00001654!]).

Figs. $31 \& 32$

Caespitose perennials. Culms 10-25 cm high erect; nodes glabrous, slightly geniculate, 1 or 2 noded. Leaf sheaths 4-10 cm long, linear, hairy; ligules thin, membranous, with a ciliate rim at apex, cilia 1-2 mm long; leaf blades $10-25 \times 0.15-0.25 \mathrm{~cm}$, linearlanceolate, flat-convolute, hairy along margins and both surfaces, acuminate to attenuate at apex. Racemes 6-12 cm long; peduncle 5-10 cm, glabrous; spikelets loosely or tightly arranged in rachis; rachis 2-3 $\mathrm{mm}$ long, triquadrate, stout, scabrid. Spikelets $5-10 \times 2-2.8 \mathrm{~mm}$, linearlanceolate, distant, dorsiventrally flattened, leaden grey, 4-6-flowered; callus bearded, hairs 1-1.2 mm long; rachilla $2 \mathrm{~mm}$ long, glabrous, almost straight, not persistent; lower glumes 2.3-3.5 × 0.5-1 mm, notched on one-side, ovate-lanceolate, 1-nerved, 1-keeled; keels slightly scabrid, acuminate or mucronate at apex, mucro $0.3 \mathrm{~mm}$ long, scabrid; upper glumes $4-5.5 \times 0.5-0.75 \mathrm{~mm}$, ellipticlanceolate, 1-nerved, 1-keeled, apex dentate or shortly awned, awns $0.5-0.8 \mathrm{~mm}$ long at sinus; lemmas 3.5-4 × 1-1.2 mm, ovate-lanceolate, coriaceous, 3-nerved, slightly keeled, 4-lobed and 3-awned; awns scabrid, apex straight or geniculate, median awn 1.5-3.6 mm long, lateral awns almost half the length of median, $0.5-2.3 \mathrm{~mm}$ long, lateral lobes ovate-acute, $0.3-1 \mathrm{~mm}$ long, margins scabrid or aristate; paleas $2-3 \times 0.5-0.75 \mathrm{~mm}$, ellipticoblanceolate, hyaline, 2-nerved, 2-keeled, keels ciliate above the halves, emarginate or 2-lobed with 


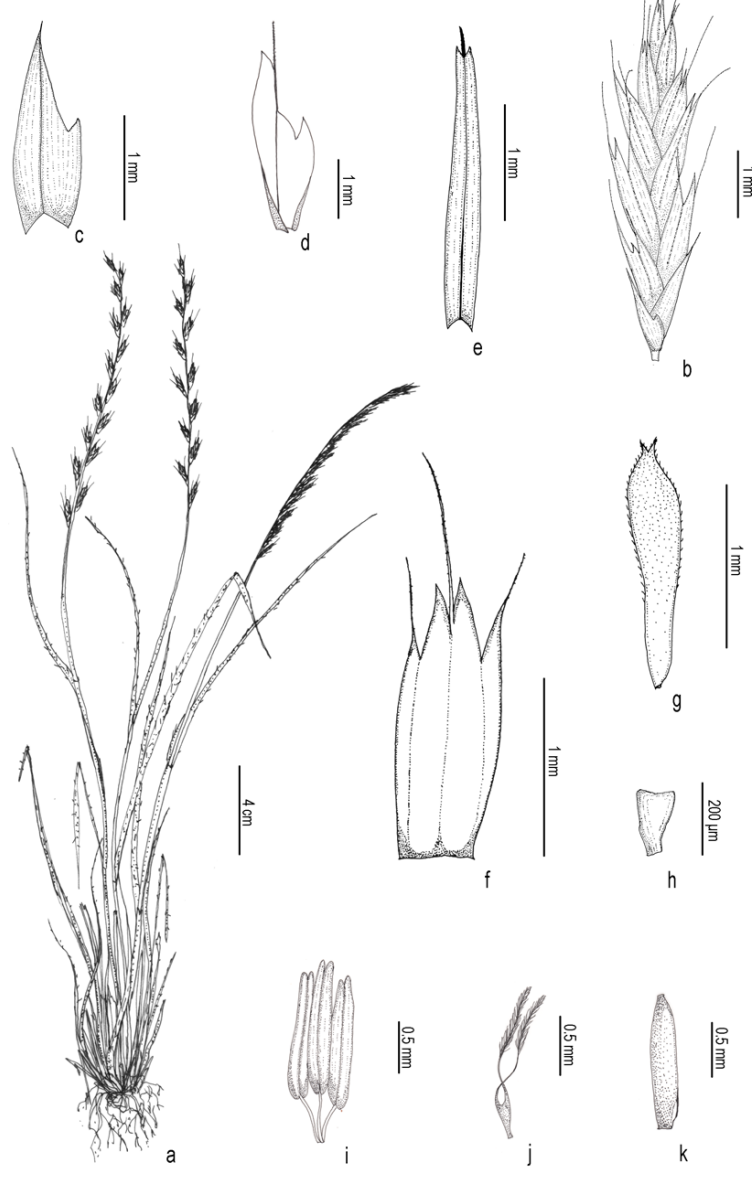

Fig. 31. Tripogon narayanae Sreek., V.J.Nair \& N.C.Nair: a. Habit; b. Spikelet; c. \& d. Lower glume; e. Upper glume; f. Lemma; g. Palea; h. Lodicule; i. Stamen; j. Pistil; k. Caryopsis.

a minute central notch at the apex; lodicules 2, 0.3$0.4 \mathrm{~mm}$ long, obovate, 3-lobed at apex; stamens 3, anthers 1.3-1.5 mm long, oblong, purple tinged, filaments 1.5-2.5 mm long, slender, glabrous; ovary 0.3-0.5 mm long, obovate, styles 2, c. $4 \mathrm{~mm}$ long, slender, hyaline, stigma 2, c. $0.6 \mathrm{~mm}$ long, plumose, creamy white. Caryopses 1.2-1.6 × 0.3-0.4 mm, narrowly oblong, light brown.

Flowering \& fruiting: Flowering and fruiting from August to October.

Habitat: This species is usually found growing along soil filled rock crevices, and wet areas along Ghat road sides and high elevation grasslands in association with Arundinella ciliata Nees, A. pumila, Garnotia tenella, Tripogon bromoides, T. munnarensis (all Poaceae), Cyanotis tuberosa (Roxb.) Schult \&
Schult.f. (Commelinaceae) and Henckelia innominata (B.L.Burtt) A.Weber \& B.L.Burtt, (Gesneriaceae).

Distribution: Endemic to Peninsular India.

Specimens examined: INDIA, Kerala, Idukki district, Kanthalloor, Pius Nagar, 13.11.2012, A.K. Pradeep \& K. Thoiba 134401a, 134401b, 134401c, 134401d; Kanthallur, 22.11.2013, A.K. Pradeep \& K. Thoiba 138008a, 138008b, 138008c, $138008 \mathrm{~d}$; Udumbanchola, Mundiyeruma, 16.12.2014, K. Thoiba \& A.K. Pradeep 144103a, 144103b, 144103c, 144103d; Chinnar Wildlife Sanctuary, 09.12.2016, A.K. Pradeep \& K. Thoiba 146764 (CALI). Tamil Nadu, Coimbatore district, Velliangiri hills, 30.09.2016, A.K. Pradeep \& Nikhil Krishna 146722, 146723 (CALI); Nilgiri district, Gudallur, suicide point, 12.12.2015, K. Thoiba 146708 (CALI); Salem district, Yercaud, Rosegarden view point, 10.12.2017, Nikhil Krishna \& K. Thoiba 146788 (CALI).

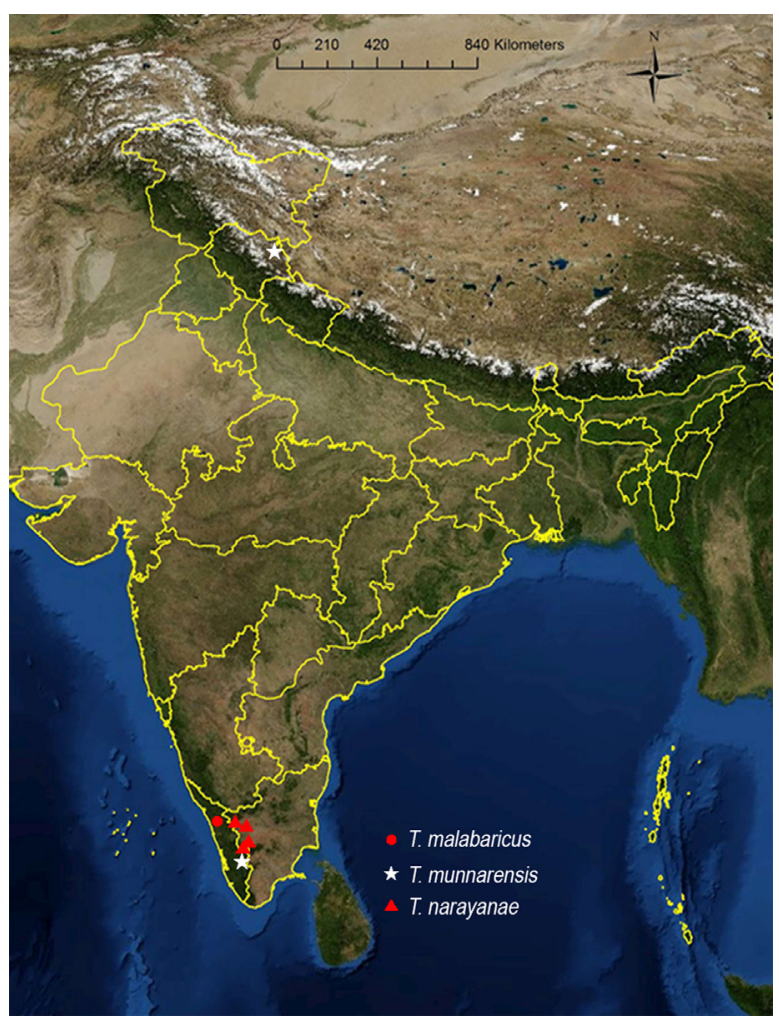

Fig. 32. Distribution of Tripogon malabaricus Thoiba \& Pradeep, T. munnarensis Thoiba \& Manudev and T. narayanae Sreek., V.J.Nair \& N.C.Nair in India [drawn using ESRI (2011) ArcGIS Desktop: Release 10]. 
Notes: It resembles Tripogon bromoides but differs in having densely tufted villous leaves, florets 4-6, lateral awns half the length of the median awns, and palea ciliate above the middle.

Tripogon polyanthus Naik \& Patunkar, Bull. Bot. Surv. India 15 (1-2): 158. 1973; S.Moulik, Grass. Bam. India 2: 623. 1997; Potdar, Salunkhe \& S.R.Yadav, Grass. Maharashtra: 563. 2012. Tripogon jacquemontii var. polyanthus (Naik \& Patunkar) Sang.Dey \& Prasanna, J. Econ. Tax. Bot. 43(1-4): 7. 2020[“2019"]. Type: INDIA, Maharashtra, Aurangabad district, Daulatabad, 18.10.1973, Patunkar 1859A (holo CAL [CAL0000002445!]; iso Marathwada University, BSI [BSI0000000677!]).

Figs. $33,34 \& 36$

Caespitose perennials. Culms 30-80 cm high, terete, erect; nodes glabrous. Leaf sheaths $1.5-6 \mathrm{~cm}$ long, ribbed, margins long ciliate; ligules membranous or ciliate; leaf blades 3-10 × $0.1-0.2$ $\mathrm{cm}$, linear, convolute, hairy at adaxially and glabrous abaxially, acuminate to attenuate at apex. Racemes 10-30 cm long, spiciform, erect or curved; spikelets arranged loosely in rachis; rachis $0.5-1.5$ $\mathrm{cm}$ long, stout, glabrous or scabridulous; peduncle $5-13 \mathrm{~cm}$, glabrous. Spikelets 2-6.5 × 0.1-0.2 cm, linear, yellowish or purple tinged, straw-coloured at dry, 20-60-flowered; callus bearded, hairs 0.5$1 \mathrm{~mm}$ long; rachilla 1-1.2 mm long, scabridulous; lower glumes 3-3.5 × 0.5-0.75 mm, notched on one-side, ovate-lanceolate, 1-nerved, 1-keeled, keels slightly scabrid, mucronulate at apex; upper glumes 3.5-4.5 × 0.75-1 mm, linear-lanceolate, 1nerved, 1-keeled, mucronate at apex, mucro 0.5 mm long; lemmas 3.5-4 × 0.6-1 mm (excluding awn), ovate-lanceolate, 3-nerved, 1-awned, 2lobed, lobes ciliate or sometimes minutely awned, median awns 1-2 mm long, scabrid; paleas 3-4.25 $\times 0.8-1.25 \mathrm{~mm}$, obovate-lanceolate, narrowly winged, hyaline, 2-keeled, keels ciliate, acute at apex; lodicules 2, 0.5-0.7 mm long, slightly cordate; stamens 3, anthers 1-1.6 mm long, oblong, filaments $0.5-0.75 \mathrm{~mm}$ long, slender, glabrous; ovary $0.3-0.5 \mathrm{~mm}$ long, obovate, styles $2,0.5-0.75$ $\mathrm{mm}$ long, slender, hyaline, stigma $0.75-1 \mathrm{~mm}$ long, plumose, creamy white. Caryopses $1.4-1.8 \times 0.2-$ $0.3 \mathrm{~mm}$, narrowly oblong-lanceolate, light brown. Flowering \& fruiting: Flowering and fruiting from August to October.

Habitat: This species is generally found growing in open situations along dry river bed rocks in association with Aristida funiculata Trin. \& Rupr., Cynodon dactylon (L.) Pers., Chrysopogon polyphyllus Blatt. \& McCann, Digitaria annиa Van der Veken, Eragrostis amabilis (L.) Wight \& Arn. (all Poaceae) and Tridax procumbens L. (Asteraceae).

Distribution: Endemic to India, hitherto known only from a few localities in Maharashtra State.

Specimens examined: INDIA, Maharashtra, Aurangabad district, Daulatabad, $400 \mathrm{~m}$, 18.10.1973, B.W. Patunkar 1857a (BSI, CAL); Nandurbar district, Tapi river bed, Prakasha, Shahada, 15.11.2016, K.V.C. Gosavi \& K. Thoiba 146747a, 146747b, 146747c (CALI); Pune district, Sinhagad, 05.09.2010, K.M. Manudev \& Santhosh Nampy 3429 (CALI).

Notes: Dey and Prasanna (2019) consider T. polyanthus as a variety of $T$. jacquemontii apparently based on the examination of herbarium specimens. However, our field studies show that they are very distinct and to be treated as two separate species. Tripogon polyanthus is rather confined to a few localities in Maharashtra and prefers to grow along dry river bed rocks, while $T$. jacquemontii is rather widespread in the hills of Central India. The report of this taxon (l.c.) from Madhya Pradesh is doubtful. Tripogon polyanthus differs from T. jacquemontii in having much longer many-flowered spikelets which are rather divaricate, more loose and strawcoloured, and the lemmas are 2-fid at the apex.

Tripogon pungens C.E.C.Fisch., Bull. Misc. Inform. Kew 1934(4): 170. 1934 \& in Gamble, Fl. Pres. Madras 3: 1834: 1934; Bor, Grass. Burma Ceylon India Pakistan 522. 1960; S.Moulik, Grass. Bam. India 2: 623. 1997; Kabeer \& V.J.Nair, Fl. Tamil Nadu Grass: 180. 2009; Potdar, Salunkhe \& S.R.Yadav, Grass. Maharashtra: 564. 2012. Type: 


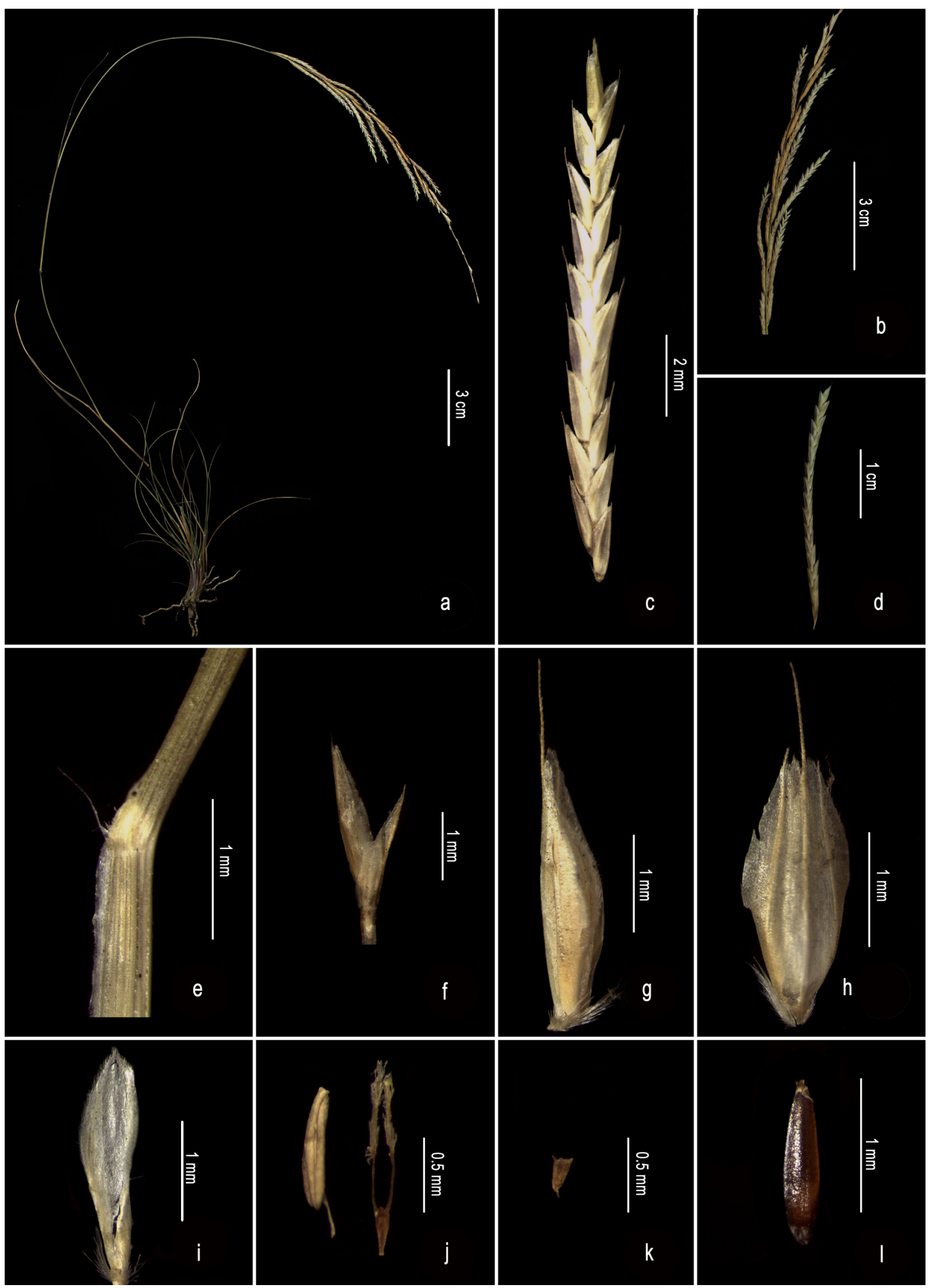

Fig. 33. Tripogon polyanthus Naik \& Patunkar: a. Habit; b. Enlarged portion of raceme; c. \& d. Spikelet; e. Ligule; f. Glumes; g. Floret; h. Lemma; i. Palea; j. Stamen \& pistil; \& k. Lodicules; I. Caryopsis. 


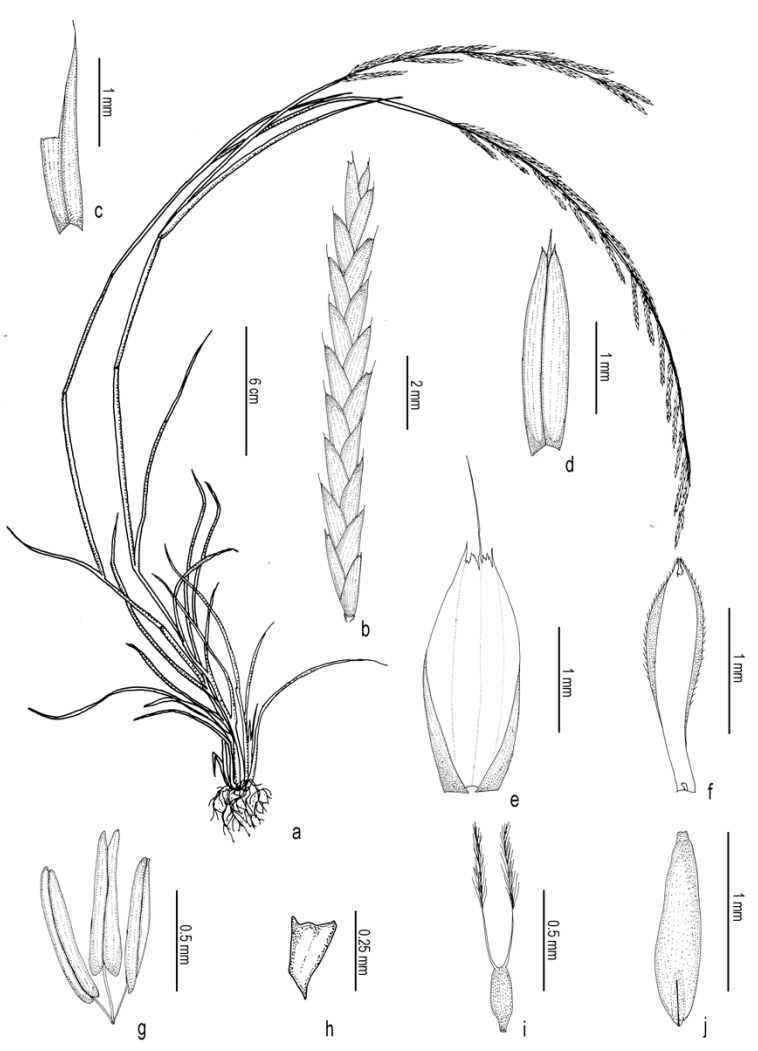

Fig. 34. Tripogon polyanthus Naik \& Patunkar: a. Habit; b. Spikelet; c. Lower glume; d. Upper glume; e. Lemma; f. Palea; g. Stamen; $\mathbf{h}$. Lodicule; i. Pistil; j. Caryopsis.

INDIA, Tamil Nadu, Coimbatore district, Punachi, Anaimalais hills, $3500 \mathrm{ft}, 10.10 .1901$, C.A. Barber 3717 (holo K [K000245044 digital image!]; iso $\mathrm{MH}[\mathrm{MH} 00002624 !])$.

Tripogon paramjitianus Murug., Arum. \& Kabeer, Indian J. Forest. 40 (3): 285. 2017, syn. nov. Type: INDIA, Tamil Nadu, Velliangiri hills, Western Ghats, 22.11.2014, Murugesan 302A (holo MH [MH00003447!]; iso MH [MH00003448!]).

Figs. $35 \& 36$

Densely tufted perennials. Culms up to $20 \mathrm{~cm}$ high, branched, thickened with the imbricating old leaf sheaths, slender, wiry and usually long exerted above; nodes concealed by the sheaths. Leaf sheaths 5-10 mm long, imbricate, striate; ligules represented by a minute hairy ridges; leaf blades $1-5 \mathrm{~cm}$ long, convolute or involute, rigid, pungent, often curved, equitant, coriaceous, 6-8 ribbed and minutely hispidulous adaxially, glabrous abaxially.
Racemes 3-8 cm long, numerous, erect or curved, 15-35 spikelets loosely or tightly arranged in rachis; rachis narrow; peduncles 5-15 cm long, glabrous. Spikelets 2-6 × 1-1.5 mm, narrowly ellipsoid, 39-flowered; callus bearded; rachilla $0.6-1 \mathrm{~mm}$ long, terete, more or less hairy, disarticulating at the base of each floret; lower glumes 2-3 × 0.5-1 mm, membranous, asymmetrical, 1-nerved, obtuse (sometimes the glume completely divided into two sub-equal parts); upper glumes $2.8-4 \times 0.5-1 \mathrm{~mm}$, oblong, acute or notched, strongly 1-ribbed, back rounded or more or less keeled; lemmas 1.5-2.5 × 0.8-1.5 mm (excluding awn), upper lemmas in spikelet tend to be smaller towards apex, broadly ovate, obtuse, 3-nerved, apex shortly 2-lobed, lobes obtuse, bearing an arista or awn from the sinus, arista 0.6-1.2 mm long; paleas 2-2.2 $\times 0.5-0.8 \mathrm{~mm}$, oblanceolate, 2-keeled, keels ciliolate, obtuse, conduplicate at apex; lodicules 2 , narrowly ob-cuneate, 0.1-0.2 mm long, truncate; stamens 3 , anthers 1 $\mathrm{mm}$ long, oblong, filaments $0.5-0.75 \mathrm{~mm}$ long, slender, glabrous; ovary minute, sub-globose, styles 2 , distinct at the base. Caryopses $0.2-0.5 \times 0.5-1$ $\mathrm{mm}$, oblong, cylindrical, light brown.

Flowering \& fruiting: Flowering and fruiting from September to November.

Habitat: This species grows on rocks in higher elevation grasslands (900-1800 m) with the lower part of the stem in rock crevices. It is found in association with Arundinella mesophylla, Jansenella griffithiana, Tripogon bromoides (all Poaceae), Eriocaulon sp. (Eriocaulaceae), Desmodium styracifolium (Osbeck) Merr. (Fabaceae) and Utricularia praeterita P. Taylor (Lentibulariaceae).

Distribution: Endemic to South India.

Specimens examined: INDIA, Kerala, Idukki district, Pothadi, Marayoor, 26.01.2018, F. Dani \& K. Thoiba 146796; Ibid., 26.01.2018, F. Dani \& K. Thoiba 158302 (CALI). Tamil Nadu, Dindigul district, s.loc., s.d., s.coll. 50215 (RHT).

Notes: Tripogon paramjitianus was described by Murugesan et al. (2017) from the Velliangiri hills in Tamil Nadu. The authors distinguished their 


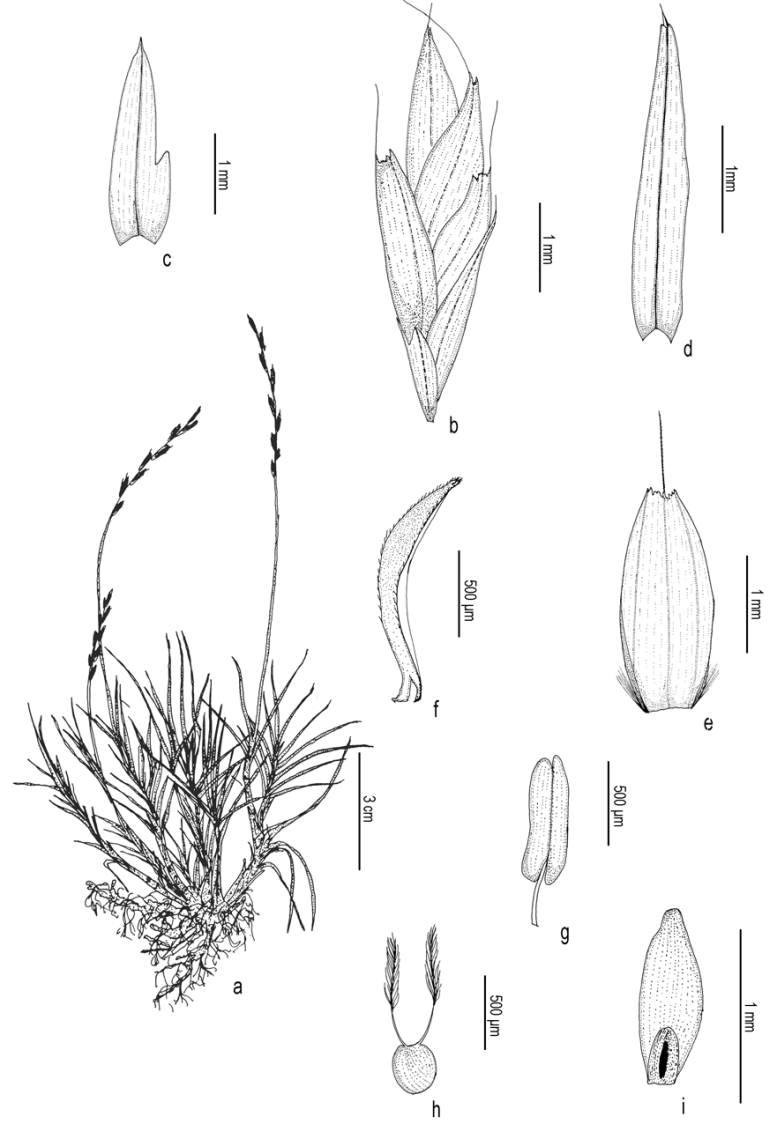

Fig. 35. Tripogon pungens C.E.C.Fisch.: a. Habit; b. Spikelet; c. Lower glume; d. Upper glume; e. Lemma; f. Palea; g. Stamen; h. Pistil; i. Caryopsis.

new species from $T$. pungens based on the size of leaf blades, lobes of glumes, and the number of florets in the spikelets. Tripogon pungens is unique in having thickened culms by persistent leaf sheaths and leaves being equitant, rigid and pungent. An examination of T. pungens in the field also shows that its leaves are not as pungent in the young stages and the racemes not much more extended from the leaves, while characters such as the size of spikelets, shape and number of lobes in glumes, size of awns in the lemma and the size of anthers are found to be variable, and cannot be taken as a reliable character in distinguishing species. An examination of the type of $T$. paramjitianus (Murugesan 302A, MH!) and other specimens from Kerala and Tamil Nadu showed it to be conspecific with T. pungens, hence reduced it here into synonymy of $T$. pungens.
Tripogon purpurascens Duthie, Ann. Roy. Bot. Gard. (Calcutta) 9: 74, t. 92. 1901; Bor, Grass. Burma Ceylon India \& Pakistan 522. 1960; S.Moulik, Grass. Bam. India 2: 623. 1997; S.M.Philips \& S.L.Chen, Kew Bull. 57(4): 913. 2002; Teerawat. \& Sungkaew, Thai For. Bull. (Bot.) 40: 130. Type: INDIA, Uttarakhand, Western Himalaya, Tehri-Garhwal district, Tons Valley, 05.05.1927, Duthie 23532 (B [B100279879 digital image!]).

Festuca filiformis Nees ex Steud., Syn. Pl. Glumac. 1: 302. 1854, non Pourr. 1788.

Tripogon abyssinicus auct. Hook.f., Fl. Brit. Ind. 7: 287. 1896, non Nees ex Steud., 1854.

Tripogon jacquemontii var. submuticus Hook.f., Fl. Brit. Ind. 7: 287. 1896. Type: INDIA, Himachal Pradesh, Simla, 08.1889, Duthie 10149 (K [K000245021 digital image!]).

Tripogon hookerianus Bor, Grass. Burma Ceylon India \& Pakistan 522. 1960. Lectotype (designated by Teerawatananon \& Sungkaew, 2012): INDIA, N.W. Himalaya, Hazara district, Kagan Valley, 8000 ft, 24.07.1896, Duthie 20364 (K [K000245025 digital image!]).

Fig. 36

Caespitose perennials, purple tinged. Culms 4-18 $\mathrm{cm}$ high, erect, sub-erect or ascending, slender, purplish, reddish-brown to green; nodes glabrous. Leaf sheaths 2-4 cm long, linear, persistent with remnants of culm bases of previous years, inrolled to the culm; ligules membranous, ciliate; leaf blades $1.7-8 \times 0.07-0.2 \mathrm{~cm}$, filiform, convolute, margins entire, densely pilose adaxially and glabrous abaxially, acuminate at apex. Racemes 3-15 cm long, solitary, spike-like, slender, straight or arcuate, spikelets arranged loosely in rachis; rachis angular; peduncles 7-11 cm long, glabrous. Spikelets $2.5-7 \times 0.4-0.5 \mathrm{~mm}$, oblong-elliptic, distant, laterally compressed, 2-4(-5-8)-flowered, purplish, with diminished sterile floret at the apex, disarticulation of florets from above glumes and in between florets; callus bearded; rachilla glabrous, almost straight, not persistent; lower glumes $1.5-3 \times 0.5-0.7 \mathrm{~mm}$, oblong-lanceolate, 


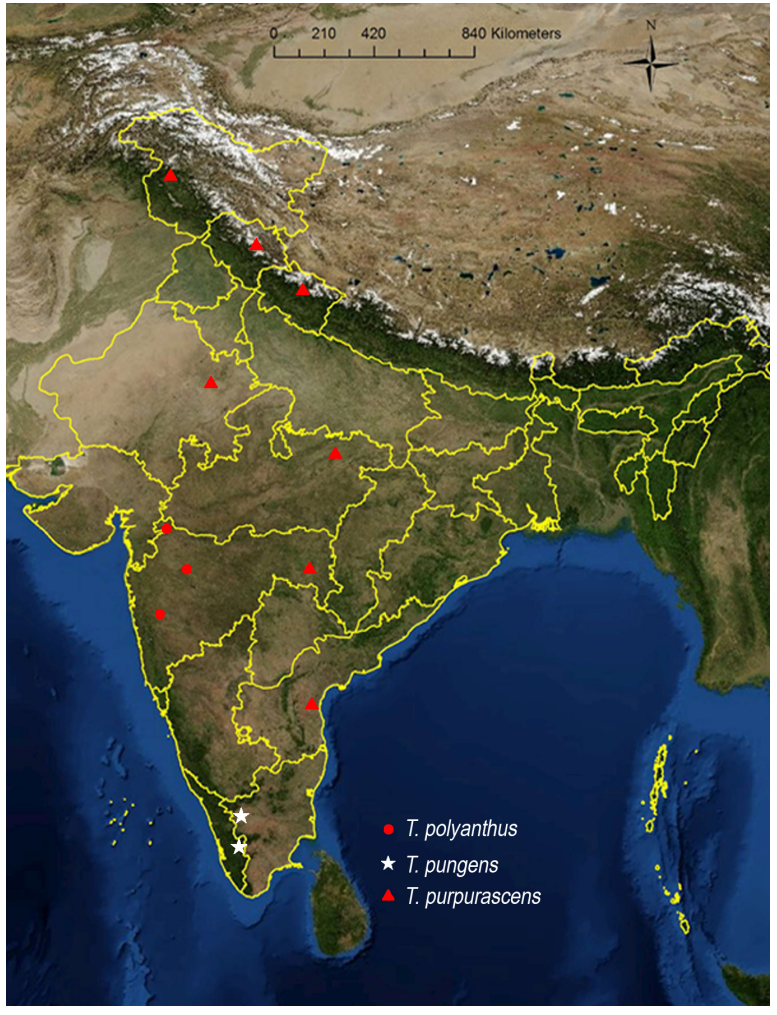

Fig. 36. Distribution of Tripogon polyanthus Naik \& Patunkar, T. pungens C.E.C.Fisch. and T. purpurascens Duthie in India [drawn using ESRI (2011) ArcGIS Desktop: Release 10].

notched on one-side, 1-nerved, 1-keeled, keels slightly scabrid, acute at apex; upper glumes (2-) 2.6-4.5 × 0.5-1 mm, elliptic-lanceolate, 1-nerved 1-keeled; lemmas 2-4.5 × 1-1.2 mm, elliptic, membranous, keeled, bifid, 3-nerved, median nerve extends to form a mucro at apex; paleas $2-4 \times 0.8-$ $1 \mathrm{~mm}$, obovate to elliptic, winged, surface pubescent, 2-keeled, ciliate along margins, obtuse or emarginated at apex; lodicules 2, 0.3-0.4 mm long, truncate; stamens 3, anthers 1-1.2 mm long, oblong, filaments $0.3-0.5 \mathrm{~mm}$ long, slender, glabrous; ovary $0.3-0.4 \times$ c. $0.25 \mathrm{~mm}$, obovate, styles 0.7-1.2 mm long, slender, hyaline, stigma 1.2-1.3 $\mathrm{mm}$ long, plumose, creamy-white. Caryopses 0.5$1.2 \times 0.2-0.3$, linear-lanceolate, light brown.

Flowering $\&$ fruiting: Flowering and fruiting from June to September.

Habitat: This species restricted to open moist, rocky areas and open grassy slopes between 750-3200 m altitudes.
Distribution: India, China, Afghanistan, Bhutan, Nepal, Pakistan, Saudi Arabia, Oman and Yemen.

Specimens examined: INDIA, Andhra Pradesh, Nellore district, Veligonda, 07.1914, M.S. Ramaswami 1417 (CAL). Himachal Pradesh, Lahasa, on the way of Shimla to Kinnaur, 19.04.2016, K.V.C. Gosavi 146798a, 146798b, 19768c (CALI). Kashmir, Ganderbal district, Sind Valley, 24.06.1892, J.F.Duthie 11457; s.loc., s.d., R.B. Majumder 670 (CAL). Madhya Pradesh, Rewa district, Nawagaon, 21.08.1954, K. Subramanyam 8565 (CAL). Maharashtra, Chandrapur district, Devada forest Camp, 20.08.1970, B.M. Wadhwa 137425 (BSI). Rajasthan, Jaipur district, Gopalpura, 17.07.1976, G.P. Roy 2679; University Campus, s.d., Y.D. Tiagi 9; s.loc., s.d., Griffith 6644; s.loc., 09.1964, Braun 3138 (CAL).

Notes: Tripogon purpurascens is unique in having all parts purplish tinged, slender racemes and 2-lobed lemma with the midrib extending in to a small mucro and awnless lateral lobes.

Tripogon ravianus Sunil \& Pradeep, Sida 19(4): 803. 2001; Kabeer \& V.J.Nair, Fl. Tamil Nadu Grass: 182. 2009. Type: INDIA, Tamil Nadu, Nilgiri district, Pykara near Udagamandalam, 1990 m, 03.12.2000, Sunil 2176 (holo BRIT!; iso CAL!, CALI [CALI112116!], K!, L!, NY!).

Tripogon bromoides Roem. \& Schult. var. major Stapf ex Hook.f., Fl. Brit. India 7(22): 288. 1896, syn. nov., non Tripogon major Hook.f., J. Proc. Linn. Soc., Bot. 7: 230. 1864. Lectotype (designated here): INDIA, Tamil Nadu, Nilgiri hills, Naduvattum, $6000 \mathrm{ft}$, 11.1888, Lawson 35 (K [K000907448 digital image!]).

Figs. $37 \& 41$

Plants caespitose perennials. Culms 24-70 cm high erect, stout; nodes glabrous, slightly geniculate. Leaf sheaths 3-10 cm long, linear, glabrous or hairy, ribbed, persistent; ligules a fine membrane with tuft of 1-4 mm long hairs; leaf blades 3-35 × 0.2-0.4 $\mathrm{cm}$, linear-lanceolate, flat-convolute, sparsely hairy and scabrid adaxially, glabrous abaxially, margins scabrid especially towards base, acuminate to attenuate at apex. Racemes 15-40 cm long, 18-48 
spikelets loosely arranged in rachis; rachis $4-7 \mathrm{~mm}$ long, stout, glabrous; peduncles $4-12 \mathrm{~cm}$ long, glabrous. Spikelets 10-25 × 2.5-3.5 mm, linear, distant, dorsiventrally flattened, leaden green, 6-14-flowered; callus bearded, hairs c. $1 \mathrm{~mm}$ long; rachilla 1-1.5 mm long, glabrous, almost straight, not persistent; lower glumes 3-4 × 1-2 mm, asymmetrical, ovate-lanceolate, 1-nerved, 1-keeled, keels slightly scabrid, unequally 2-lobed, acuminate at apex; upper glumes 5-6 × 1.5-2 mm, ellipticlanceolate, 1-nerved, 1-keeled, coriaceous, lobed and awned at sinus, awns 1-1.5 mm long, scabrid, lobes $0.5-1 \mathrm{~mm}$ long; lemmas 3-4 × 2-3 mm (excluding awn), elliptic-lanceolate, coriaceous, 3nerved, 4-lobed, slightly keeled, 3-awned, awns scabrid, straight, median awns 4-5 mm long, lateral awns 3-3.5 mm long, lateral lobes $2 \mathrm{~mm}$ long, lanceolate-acuminate; paleas 2.5-3.5 × 1-1.5 mm, elliptic-oblanceolate, hyaline, winged, 2-keeled; keels ciliate or 2-lobed, acute with a minute central notch at apex; lodicules 2, 0.5-0.75 mm long, wedge shaped; stamens 3 , anthers 1.5-2 mm long, oblong, filaments 1-1.5 mm long, slender, glabrous; ovary $0.75-1 \times 0.5 \mathrm{~mm}$, obovate, styles $2, c .1 \mathrm{~mm}$ long, slender, hyaline, stigma 1-1.5 mm long, plumose, creamy white. Caryopses $1.8-2.3 \times 0.4-$ $0.7 \mathrm{~mm}$, oblong-cylindric, light brown.

Flowering \& fruiting: Flowering and fruiting from September to late December.

Habitat: This species grows from 1500-2200 m elevations on road cuts and seasonally wet rocky hillsides. It is found growing in association with Anaphalis leptophylla DC. (Asteraceae), Eragrostis collinensis, E. nigra Steud., E. schweinfurthii Chiov., Tripogon bromoides (all Poaceae), Hypericum mysurense Wight \& Arn. (Hypericaceae) and Spermacoce articularis L.f. (Rubiaceae).

Distribution: Endemic to South India.

Specimens examined: INDIA, Kerala, Idukki district, Valakettimala, Moolamattam, 09.09.1998, C.N. Sunil 2115a, 2115b (CALI); Palakkad district, Silent Valley National Park, 25.09.2013, K. Thoiba \& K.M. Manudev 135132; Nelliyampathy, Kurishumudi, s.die., C.N. Sunil 4478 (CALI). Tamil Nadu, Coimbatore district, Velliangiri hills, 22.02.1932, S.R. Raju \& Ratnavelu 250 (MH); Ibid., 30.09.2016, A.K. Pradeep, Nikhil Krishna \& K. Thoiba 146768 (CALI); Nilgiri district, Emerald, 09.11.2012, Remya J. \& Prasanna 75485 (TBGT); Gudallur, shooting point, 12.12.2015, K. Thoiba 146704; Naduvattam, 10.1910, Bor 11562 (CAL); Ibid., 23.02.2010, Santhosh Nampy 2778 (CALI); Ibid., s.d., s.coll. 56 (MH); Nilgiri hills, Coonor, 20.03.1870, s.coll.11125a; Ibid., 20.03.1870, s.coll.11125b (CAL); Pykara, 03.12.2000, C.N. Sunil 2176, 2163; Suicide point, 12.12.2015, K. Thoiba 146707; (CALI); Udagamandalam, 23.12.2017, Nikhil Krishna \& K. Thoiba 146794 (CALI); Ibid., 12.09.1930, N. Narayanaswami 4190, 4273; Ibid., 09.01.1902, C.A. Barber 4161a, 4161b (MH).

Notes: Hooker (1896) recognized a new variety, $T$.
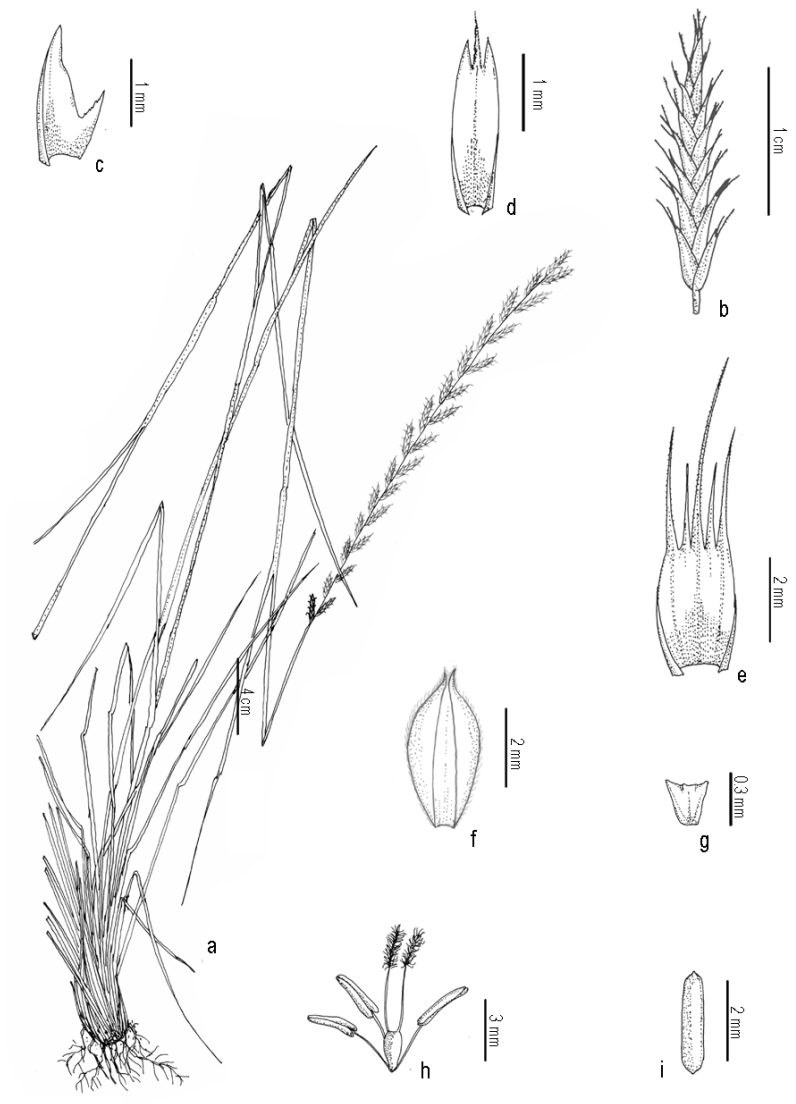

Fig. 37. Tripogon ravianus Sunil \& Pradeep: a. Habit; b. Spikelet; c. Lower glume; d. Upper glume; e. Lemma; f. Palea; g. Lodicule; h. Stamen \& pistil; i. Caryopsis. 
bromoides var. major based on a specimen (Lawson 35, K000907448 digital image!) collected from Naduvattam, Nilgiri hills of South India, which is treated here as conspecific with T. ravianus described from Nilgiri hills.

Tripogon sivarajanii Sunil, Sida 18(3): 809.1999; Sasidh., Biodiv. Doc. Kerala-Fl. Pl.: 595. 2004. Type: INDIA, Kerala, Idukki district, Valakettimala near Moolamattam, 1020 m, 02.09.1997, C.N. Sunil 2117 (holo MH!; iso BRIT!, CALI [CALI112132!], K!, L!, PBL!).

Tripogon jayachandranii Arum. \& Murugan, Indian J. Forest. 40(2): 159. 2017, syn. nov. Type: INDIA, Tamil Nadu, Theni district, Meghamalai Wildlife Sanctuary, 1450 m, 04.10.2016, S. Arumugam \& C. Murugan 134462 (holo CAL!; iso $\mathrm{MH}$ [MH00003331!]).

Figs. $38,39 \& 41$

Tufted perennial herb. Culms 30-70 cm high erect; nodes glabrous. Leaf sheaths $6-10 \mathrm{~cm}$ long, linear, glabrous; ligules a fine glabrous membrane; leaf blades $10-40 \times 0.2-0.3 \mathrm{~cm}$, linear-lanceolate, flatconvolute, villous adaxially with short dense hairs intermingled with long scattered shaggy hairs, glabrous abaxially, acuminate to attenuate at apex. Racemes 10-45 cm long, stout, 20-60 spikelets appressed to concavities in rachis; rachis 3-7 mm long, stout, glabrous; peduncle 3-9 cm, glabrous. Spikelets 7-10.5 × 1.5-2 mm, linear, distant, dorsiventrally flattened, 5-8-flowered; callus hairy, hairs c. $0.5 \mathrm{~mm}$ long; rachilla $0.5-1 \mathrm{~mm}$ long, glabrous, straight or zig zag, not persistent; lower glumes $2-2.5 \times 0.5-1 \mathrm{~mm}$, asymmetrical, oblonglanceolate, 1-nerved, 1-keeled, keels slightly scabrid, acuminate at apex; upper glumes 3-4.2 $\times$ 0.5-1 mm, elliptic-lanceolate, 1-nerved, 1-keeled, acuminate or mucronulate at apex, lemmas $3-4 \times$ 1.5-2 mm, oblanceolate, 2-lobed, 3-nerved, slightly keeled, 1-awned, awns stout, scabrid, straight, 3-3.5 mm long, lateral lobes $0.8-1.5 \mathrm{~mm}$ long; paleas 2-3 × 1-1.5 mm, obovate-elliptic, narrowly winged, hyaline, 2-keeled, keels ciliolate, acute or 2-lobed with a minute central notch at apex; lodicules 2, c. $0.5 \mathrm{~mm}$ long, slightly cordate;

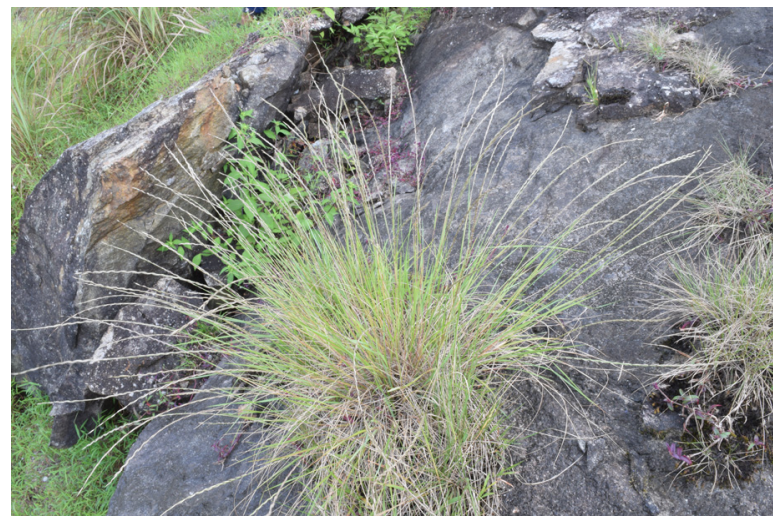

Fig. 38. Tripogon sivarajanii Sunil in its natural habitat at Vagamon in Idukki district (Photo by K. Thoiba)

stamens 3, anthers 1-1.5 mm long, oblong, filaments $0.5-0.75 \mathrm{~mm}$ long, slender, glabrous; ovary $0.25-0.4 \mathrm{~mm}$ long, obovate, styles 2 , 0.5$0.75 \mathrm{~mm}$ long, slender, hyaline, stigma 0.75-1.2 $\mathrm{mm}$ long, plumose, creamy white. Caryopses 1.2$1.6 \times 0.3-0.4 \mathrm{~mm}$, narrowly oblong, cylindric, light brown.

Flowering \& fruiting: Flowering and fruiting from September to early December.

Habitat: This species is usually found growing along grassy hill slopes at 800-1800 m elevations in association with Arundinella mesophylla, Chrysopogon hackelii, Eragrostis atrovirens (Desf.) Trin. ex Steud., E. collinensis, Eragrostiella bifaria, Tripogon bromoides (all Poaceae), Crotalaria grahamiana Wight \& Arn. (Fabaceae), Henckelia fischeri (Gamble) A. Weber \& B.L. Burtt, $H$. gambleana (C.E.C.Fisch.) A.Weber \& B.L. Burtt (both Gesneriaceae), Impatiens acaulis (Balsaminaceae), Justicia latispica (Clarke) Gamble (Acanthaceae), Murdannia semiteres (Commelinaceae) and Swertia corymbosa (Griseb.) Wight ex Clarke (Gentianaceae).

Distribution: Endemic to South India.

Specimens examined: INDIA, Kerala, Ernakulam district, Shoolamudi, Variyam, 14.12.2016, Nikhil Krishna \& K. Thoiba 146741; Ibid., 14.12.2016, Nikhil Krishna \& K. Thoiba 146743 (CALI); Idukki district, Kattapana, 16.12.2014, K. Thoiba \& A.K. Pradeep 144101; Kalvari Mount, Kattapana, 17.10.2015, K. Thoiba \& A.K. Pradeep 146634; 


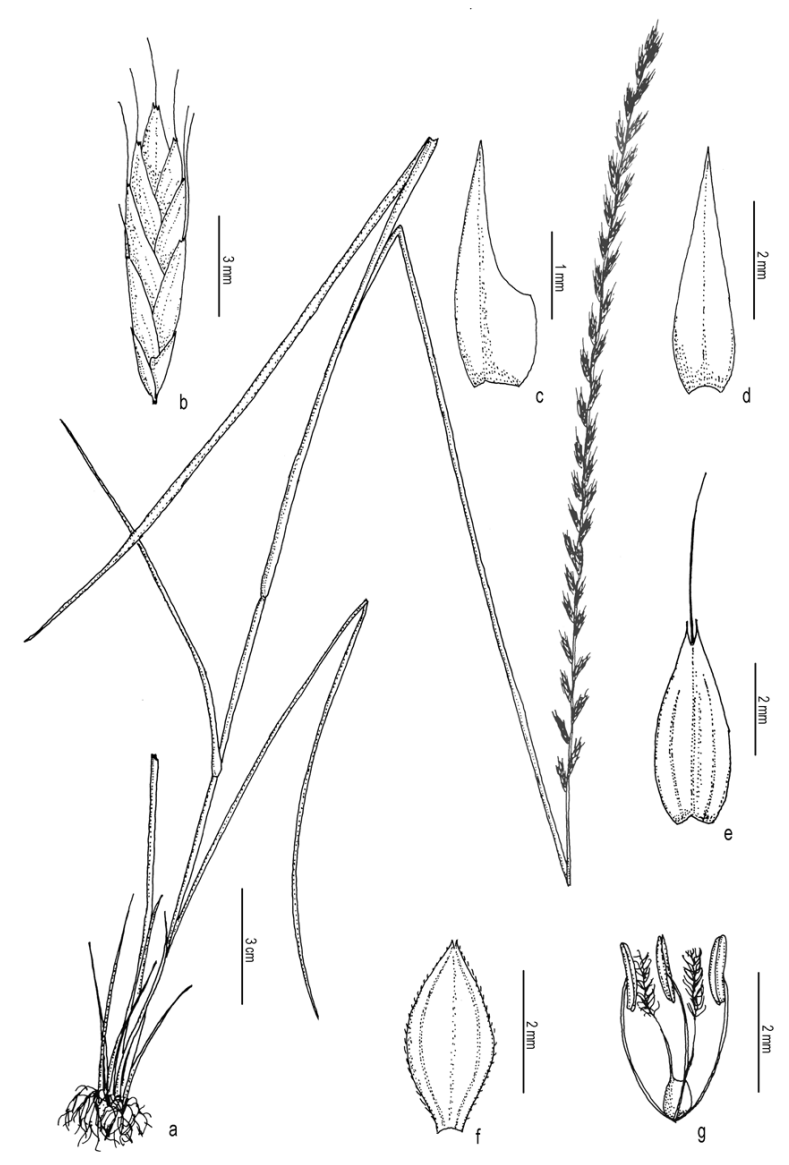

Fig. 39. Tripogon sivarajanii Sunil: a. Habit; b. Spikelet; c. Lower glume; d. Upper glume; e. Lemma; f. Palea; g. Stamen \& pistil.

Kuttikanam Mundakayam road, 15.10.2015, K. Thoiba \& A.K. Pradeep 146607; Marayoor, 24.05.2016, T. Shaju 66748; Munnar, Devikulam, 16.12.2014, K. Thoiba 137583; Meenuliyampara, 23.12.2009, Santhosh Nampy 2735; Peerumade, 16.10.2015, K. Thoiba \& A.K. Pradeep 146614; Parunthpara, K. Thoiba \& A.K. Pradeep 146632; Periyakanal waterfalls, 14.11.2014, K. Thoiba \& A.K. Pradeep 137547; Vagamon, way to Pullikanam, 15.10.2015, K. Thoiba \& A.K. Pradeep 146610; Ibid., 15.10.2015, K. Thoiba \& A.K. Pradeep 146612; Valakettimala near Moolamattam, 02.09.1997, C.N. Sunil 2117 (CALI); Kollam district, Varkala, Sivagiri, Jadayipara, 10.10.2015, K. Thoiba \& P. Soumya 146602 (CALI); Kottayam district, s.loc., 05.11.1956, S.C. Agarwal 881 (CALI); Palakkad district, Nelliyampathy, Keshavanpara, 07.11.2013, K. Thoiba 134485; Ibid., 07.11.2015, K. Thoiba
146678 (CALI); (CALI); Thiruvananthapuram district, Bonacaud, s.d., N. Mohanan 11389 (TBGT); Ponmudi, 05.09.2017, Nikhil Krishna \& K. Thoiba 146755 (CALI); Thrissur district, Parambikulam Tiger Reseve, Karimala hills, 26.09.2013, K. Thoiba 134447, 134451; Shaekalmudi, Mudiyankunnu, 12.12.2013, K. Thoiba 138027 (CALI). Tamil Nadu, Coimbatore district, Valparai, Shivamalai, 13.12.2013, K. Thoiba 138010 (CALI).

Notes: Arumugam and Murugan (2017) described Tripogon jayachandranii Arum. \& Murugan from the Meghamalai Wildlife Sanctuary in Tamil Nadu, India, and compared it with $T$. jacquemontii, $T$. pungens, T.copei, and T. wightii. According to them, these four species are allied to $T$. jayachandranii. During the course of the present investigation, the authors of the present revision have examined the types and protologues of all the five taxa involved, and found that, T. jayachandranii is conspecific with $T$. sivarajanii Sunil and not at all similar to any one of the species Arumugam and Murugan (2017) compared with, and hence it is reduced to synonymy of $T$. sivarajanii.

Tripogon trifidus Munro ex Stapf, Bull. Misc. Inform. Kew 1892(64): 85. 1892; Hook.f., Fl. Brit. India 7(22): 286. 1896; Bor, Grass. Burma Ceylon India \& Pakistan 524. 1960; S.Moulik, Grass. Bam. India 2: 623. 1997; Potdar, Salunkhe \& S.R.Yadav, Grass. Maharashtra: 564. 2012. Lectotype (designated by Noltie, 1999): INDIA, Meghalaya, Khasia hills, alt. 4-5500 ft., s.d., Griffith 6634 (K [K000245010 digital image!]).

Tripogon tirumalae Chorghe, Rasingam, Prasanna \& Sankara Rao, Phytotaxa 131(1): 17. 2013, syn. nov. Type: INDIA, Andhra Pradesh, Tirumala hills, near Kumaradara Pusupudara Dam, N 13 43.509', E 79²19.345', 990 m, 04.09.2012, L. Rasingam, M.S. Rao \& Alok Chorghe 2914 (holo CAL [CAL0000025198!]; iso BSID!).

Tripogon umae-ganeshii B.R.P.Rao \& M.Anil Kumar, Indian J. Forest. 41(1): 97. 2018, as "uma-ganeshii", syn. nov. Type: INDIA, Andhra Pradesh, Horsely hills, N $13^{\circ} 38^{\prime} 44.0^{\prime}$, E $78^{\circ} 24^{\prime} 50.1^{\prime \prime}, 1195$ m, 
19.09.2016, B. Ravi Prasad Rao \& Midigesi Anilkumar 51980 (holo SKU digital image!; iso BSID).

Tripogon nallamalayanus Rasingam \& J.Swamy, Phytotaxa 351(4): 296. 2018, syn. nov. Type: INDIA, Telangana, Mahbubnagar district, Umamaheshwaram, N 16³6'989", E 78 73'168", 700 m, 22.10.2016, L. Rasingam \& J. Swamy 7396 (holo CAL!; iso BSID [BSID0016571digital image!]).

Figs. $40 \& 41$

Caespitose perennials. Culms 20-95 cm high; nodes glabrous. Leaf sheaths 4-12 cm long, linear, glabrous, persistent, inrolled to the culm; ligules a densely ciliate membrane, cilia 0.3-1.2 mm long, often glabrous when dry; leaf blades 10-70 × 0.1$0.2 \mathrm{~cm}$, linear-lanceolate, filiform, involute, ribbed, sparsely hairy adaxially and glabrous abaxially, margins scabrid, acuminate to attenuate at apex. Racemes 5-35 cm long, solitary, spike like, spikelets loosely or tightly arranged in rachis; rachis angular; peduncle 7-25 cm long, glabrous. Spikelets 4-16× 0.7-2 mm, elliptic, greenish to yellow, 5-20flowered, disarticulation of florets from above glumes and in between florets; callus bearded; rachilla $0.3-0.5 \mathrm{~mm}$ long, not persistent; lower glumes 2-4 × 0.5-0.7 mm, lanceolate, asymmetrical, 1-nerved, 1-keeled, acuminate, attenuate or mucronulate at apex, unlobed at maturity; upper glumes 3-5 × 1-1.2 mm, lanceolate, 1-nerved, 1keeled, apex 2-toothed at maturity, mucronate or minutely awned at sinus; lemmas 3-4.5 × 1-1.3 mm (excluding awn), elliptic-lanceolate, 2-lobed, 3nerved, 3-awned or not, median awns $2.8-4.5 \mathrm{~mm}$ long, scabrid, slightly geniculate, flexuous or recurved, lateral lobes acute to acuminate or sometimes lateral veins extended to lateral awns, awns 0.2-0.5 mm long; paleas 2-2.6 $0.5-0.6 \mathrm{~mm}$, obovate-elliptic, narrowly winged, hyaline, 2keeled, 2-nerved, ciliate along the margins, apex 2-lobed or bidentate; lodicules 2, 0.1-0.2 mm long, 2-toothed at apex; stamens 3, anthers $0.5-0.7 \mathrm{~mm}$ long, oblong, filaments $0.3-0.4 \mathrm{~mm}$ long, slender; ovary $0.2-0.3 \times$ c. $0.25 \mathrm{~mm}$, obovate, styles $0.3-0.4$ $\mathrm{mm}$ long, slender, hyaline, stigma c. $0.5 \mathrm{~mm}$ long, plumose, creamy white. Caryopses 1.6-2.5 × 0.25-
$0.3 \mathrm{~mm}$, elliptic-lanceolate, dark brown.

Flowering \& fruiting: Flowering and fruiting from September to October.

Habitat: Rare on rocky hill slopes, it grows on rocks at elevations between 800 to $2500 \mathrm{~m}$, in association with Chamaecrista mimosoides (L.) Greene. (Fabaceae), Apluda mutica, Digitaria longiflora (Retz.) Pers., Eragrostiella bifaria, E. brachyphylla (Stapf) Bor, Heteropogon contortus (L.) Roem. \& Schult., Ischaemum sp., Lophopogon tridentatus Hack., Tripogon bromoides and T. wightii (all Poaceae).

Distribution: India, Nepal, Bhutan, Myanmar, China, Thailand and Vietnam.

Specimens examined: INDIA, Andhra Pradesh, Chittor district, Arai, Nagalakona, 23.02.2017, K. Thoiba \& Mohammed Yoonus 146781; Penchalakona hills, 08.09.2016, Mohammed Yoonus, Nikhil Krishna \& K. Thoiba 146782; Talakona watch house,

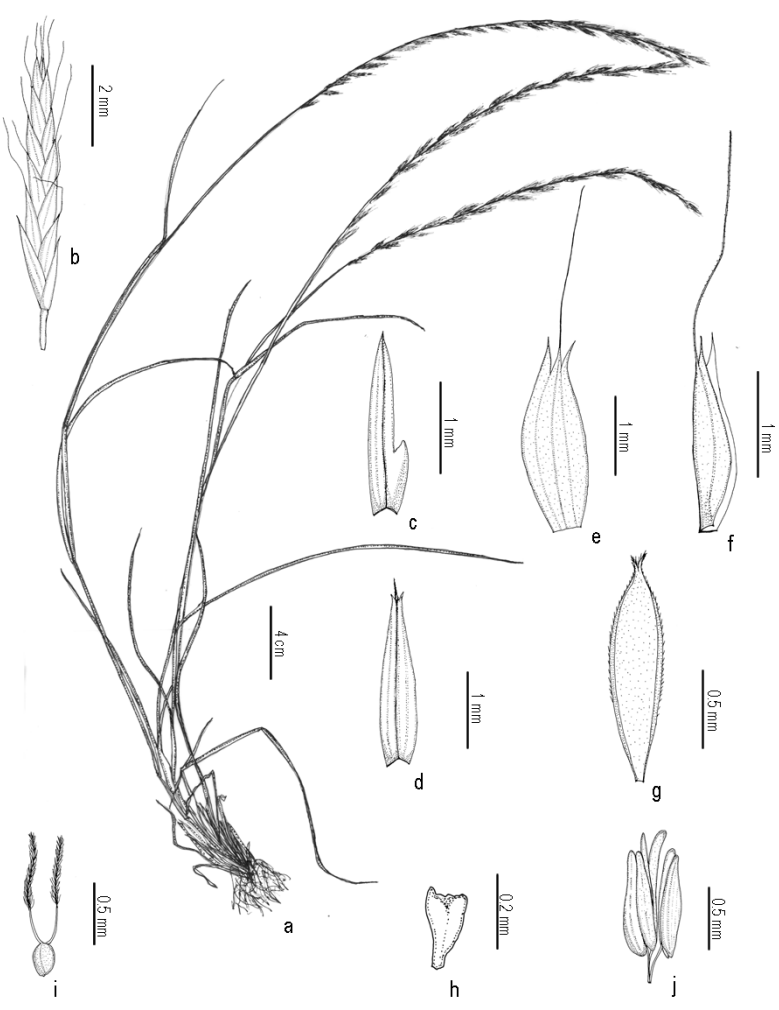

Fig. 40. Tripogon trifidus Munro ex Hook.f.: a. Habit; b. Spikelet; c. Lower glume; d. Upper glume; e. \& f. Lemma; g. Palea; h. Lodicule; i. Pistil; j. Stamen. 


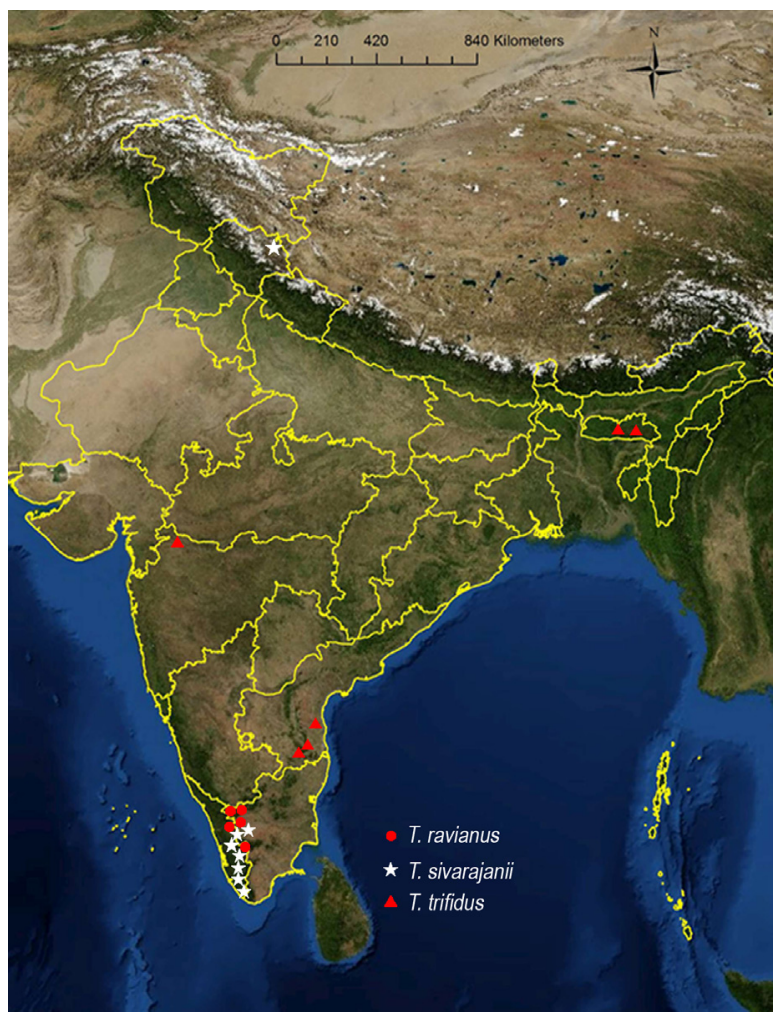

Fig. 41. Distribution of Tripogon ravianus Sunil \& Pradeep, T. sivarajanii Sunil and T. trifidus Munro ex Hook.f. in India [drawn using ESRI (2011) ArcGIS Desktop: Release 10].

09.12.2017, Soumya, Geethika \& K. Thoiba 146791 (CAL); Penchalakona hills, s.d., s.coll. 146782 (CALI); Nellore district, Rapur Ghat, 09.10.2016, B.R.P. Rao \& M. Anil Kumar 52148 (SUK). Maharashtra, Dhule district, Tarad, near Shirpur, 17.09.2015, K.V.C. Gosavi \& K. Thoiba 146784 (CALI). Meghalaya, East Khasi hills district, Cherrapunjee, 07.1878, G. Gallatly 476; K hills, 09.1856, s.coll. s.n.; K \& J hills, 22.12.1959, G. Panigrahi 19282; Ibid., 24.09.1886, C.B. Clarke 45089; Khasia, 25.09.1886, C.B. Clarke 45671A; Ibid., Kullong, 23.08.1885, C.B. Clarke 40028; Ibid., 29.08.1886, C.B. Clarke 45092; Mawphlang, 11.09.1886, C.B. Clarke 44707A; Shillong, Bishop Falls, 26.09.1885, C.B. Clarke 40543; s.loc., 08.1919, P.W. Meekinnon s.n. (CAL).

Notes: Tripogon trifidus is extremely variable. Specimens from northeast and southern India often look different. The height of the plant varies from 20-95 cm, the ligules are very short and ciliate at maturity, but at the flowering stage, long pilose hairs may be present at the throat, which fall down when reaching maturity. Both awned and unawned types of lemmas (from young and mature racemes) are seen in a single collection.

Tripogon tirumalae was described by Chorghe et al. (2013) based on collections from Tirumala hills in Andhra Pradesh. The authors distinguished $T$. tirumalae from T. trifidus by its height $(60-75 \mathrm{~cm})$, glabrous leaf sheaths near the tip, unawned lemma lobes, median awns being 1.5 times longer than the lemma. An examination of T. trifidus specimens available in Indian herbaria, along with live collections from Tirumala and adjacent hill ranges in Chittoor district of Andhra Pradesh revealed that T. trifidus is a highly variable taxon. An examination of the type specimens (L. Rasingam, M.S. Rao \& Alok Chorghe 2914) available at CAL and BSID and a number of collections from the type locality also revealed the characters used to distinguish $T$. tirumalae from $T$. trifidus are rather variable and cannot be relied upon. Hooker (1896) had also observed that the lobes of the lemma are "awned or irregularly toothed" in T. trifidus. The length of the median awn of the lemma also ranges from 2.8 $\mathrm{mm}$ to $4.5 \mathrm{~mm}$. Examination of the types of both the taxa also support the merging of Tripogon tirumalae under T. trifidus. The other two recently described species T. nallamalayanus Rasingam \& J.Swamy and T.umae-ganeshii B.R.P.Rao \& M.Anil Kumar also belong to T. trifidus. Rasingam and Swamy (2018) distinguished T. nallamalayanus from T. trifidus by its glabrous leaf sheaths, longer spikelets and geniculate awn on the lemma. These characters are also variable and cannot be relied upon for their segregation. The leaf sheath of $T$. trifidus is always pubescent when young, later the hairs fall off and the sheath appears glabrous. The length of the spikelets varies from 4 to $16 \mathrm{~mm}$, and the awn may be straight, wavy or shortly geniculate in this species. According to Rao and Anil Kumar (2018) Tripogon umae-ganeshii differs from T. trifidus in having longer $(30-70 \mathrm{~cm})$ leaf blades, glabrous leaf sheath, more florets (13-20) in the spikelets. 
All these characters are also shared by T. trifidus, and hence the names, T. nallamalayanus and $T$. umae-ganeshii are reduced to synonymy of $T$. trifidus.

Tripogon vellarianus Pradeep, Sida 18(3): 811. 1999; Sasidh., Biodiv. Doc. Kerala-Fl. Pl.: 595. 2004. Tripogon major Hook.f. subsp. vellarianus (Pradeep) Sang.Dey \& Prasanna, J. Econ. Tax. Bot. 43(1-4): 9. 2020[“2019”]. Type: INDIA, Kerala, Kozhikode district, Vellarimala, $11^{0} 25.877^{\prime} \mathrm{N}, 76^{\circ}$ 06.765’ E, 1300 m, 17.10.1997, A.K. Pradeep 56110 (holo MH!; iso BRIT!, CAL!, CALI [CALI112131!], K!, L!, NY [NY00658707 digital image!] PBL!).

Figs. $42,43 \& 50$

Tufted perennial herb. Culms 30-150 cm high erect; nodes glabrous. Leaf sheaths $4-10 \mathrm{~cm}$ long, linear, glabrous; ligules indistinct or a tuft of 1-4 $\mathrm{mm}$ long hairs at apex in young stage; leaf blades 30-60 × 8-15 mm, linear-lanceolate, flat, sparsely hairy at adaxially and glabrous abaxially, acuminate to attenuate at apex. Racemes 30-40 cm long, Spikelets appressed to concavities in rachis; rachis $2.5-3 \mathrm{~cm}$ long, rigid, glabrous; peduncle $10-25 \mathrm{~cm}$ long, glabrous. Spikelets 10-17 × 3-3.6 mm, linear, distant, dorsiventrally flattened, 6-12-flowered; callus bearded, hairs 1-1.2 mm long; rachilla 0.5 $\mathrm{mm}$ long, glabrous, straight, not persistent; lower glumes $4-5.2 \times 0.9-1.1 \mathrm{~mm}$, lanceolate, symmetrical, 1-nerved, 1-keeled, glabrous, purplish tinged dorsally, acuminate at apex; upper glumes 5-7.5 × 1-1.5 mm, elliptic-lanceolate, 1-keeled, 13-nerved, glabrous acute to acuminate at apex; lemmas 5-10 × 1-2 mm (excluding the awn), ovatelanceolate, 3-nerved, slightly keeled, 1-awned, awns scabrid, apex straight or geniculate, median awns $3 \mathrm{~mm}$ long, awns scabrid, lateral awns absent or closely adpressed to the median awn; paleas 4-8 $\times 0.8-1 \mathrm{~mm}$, narrowly elliptic, hyaline, 2-keeled, winged, keels minutely puberulous, acute or obtuse at apex; lodicules 2, 0.25-0.5 mm long, quadrate, apex coarsely 3-toothed; stamens 3, anthers 1.5-2 $\mathrm{mm}$ long, oblong, filaments $0.5 \mathrm{~mm}$ long, slender, glabrous; ovary $0.25 \mathrm{~mm}$ long, obovate, styles 2 , $0.8 \mathrm{~mm}$ long, slender, hyaline, stigmas $0.6-0.8 \mathrm{~mm}$ long, plumose, creamy white to purplish. Caryopses 2.5-3.5 $\times$ 0.5-0.7 mm, oblong-cylindric, light brown.

Flowering \& fruiting: Flowering and fruiting from September to January.

Habitat: This species is rather rare and occurs from 1000 to $1500 \mathrm{~m}$ on seasonally wet rocky hill sides in association with Arthraxon lancifolius, Arundinella pumila, Chrysopogon nodulibarbis, Themeda tremula, Tripogon bromoides (all Poaceae), Cyanotis arachnoidea C.B.Clarke, Murdannia semiteres (both Commelinaceae), Henckelia humboldtiana (Gesneriaceae) and Ipsea malabarica Hook.f. (Orchidaceae).

Distribution: Endemic to South India, hitherto known only from the type locality and adjoining areas.

Specimens examined: INDIA, Kerala, Kozhikode

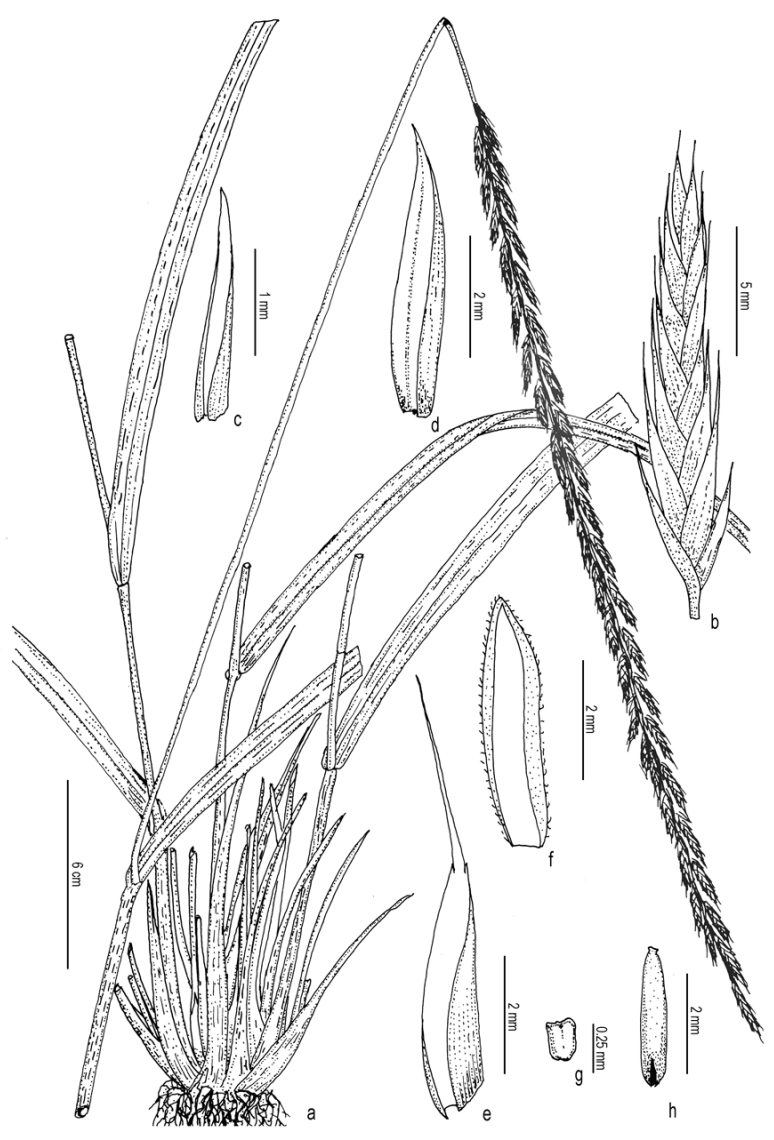

Fig. 42. Tripogon vellarianus Pradeep: a. Habit; b. Spikelet; c. Lower glume; d. Upper glume; e. Lemma; f. Palea; g. Lodicule; h. Caryopsis. 


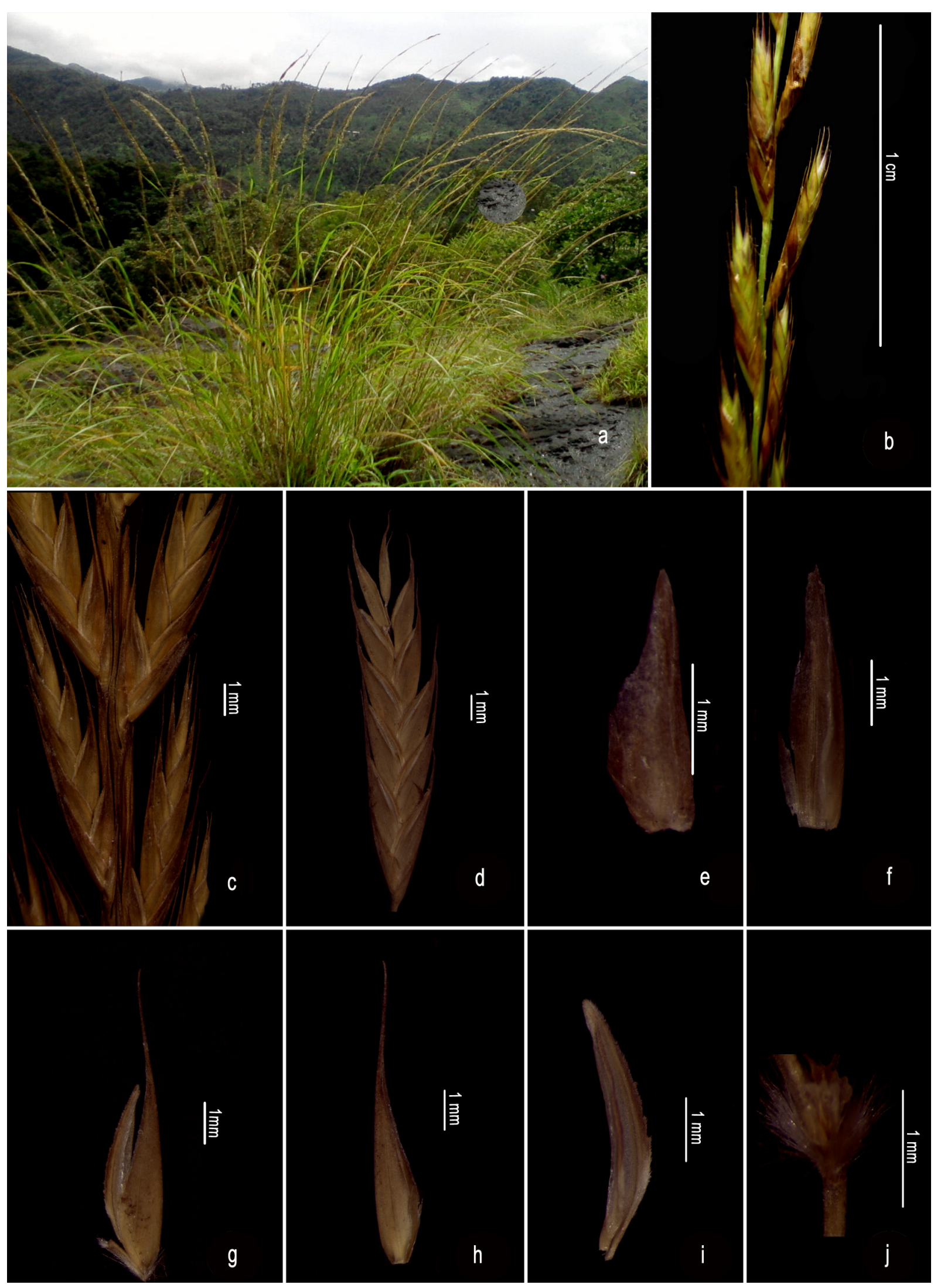

Fig. 43. Tripogon vellarianus Pradeep: a. Habit; b. \& c. Enlarged portion of raceme; d. Spikelet; e. Lower glume; f. Upper glume; g. Floret; h. Lemma; i. Palea; j. Callus with lodicules. 
district, Damodaran kolli, way to Vellarimala, 14.11.1997, A.K. Pradeep 56187a, 56187b, 56187c; above Olichuchattum falls, way to Vellarimala, 19.98.1997, A.K. Pradeep 56035a, 56035b, 56035c; Kakkadampoyil, 09.10.2013, K. Thoiba 134468a, 134468b, 134468c (CALI).

Notes: Dey and Prasanna (2019) without having studied the living populations in the field, treated this taxon as a subspecies of the South African $T$. major Hook.f. Tripogon vellarianus is a distinct species characterised by its strongly tussockforming habit, broad leaves and large spikelets. It is perhaps the tallest species of Tripogon in the genus.

Tripogon velliangiriensis Murug. \& V.Balas., Indian J. Forest. 31(1): 109. 2008; Kabeer \& V.J.Nair, Fl. Tamil Nadu Grass: 182. 2009. Type: INDIA, Tamil Nadu, Coimbatore district, Velliangiri hills, 1850 m, 12.09.2003, Murugesan KASCH1181 (holo KASCH; iso $\mathrm{MH}$ [MH00003233!]).

Tripogon copei Newmaster, V.Balas., Murug. \& Ragup., Syst. Bot. 33 (4): 698. 2008. syn. nov. Type: INDIA, Tamil Nadu, Velliangiri hills, $1840 \mathrm{~m}$, 18.12.2006, Ragu \& Newmaster 55277 (holo OAC; iso $\mathrm{KASCH})$.

Tripogon wightii Hook.f. var. kanyakumariensis Kabeer \& V.J.Nair, Fl. Tamil Nadu Grass. 183. 2009. syn. nov. Type: INDIA, Tamil Nadu, Kanyakumari district, Mothiramalai, Thomarai, near Peechiparai, $\pm 630 \mathrm{~m}, 16.12 .2005$, K. Althaf Ahamed Kabeer 118769 (holo \& iso MH [MH00003228!]).

Figs. $44,45 \& 50$

Caespitose perennials. Culms 10-25 cm high, erect or stoloniferous; nodes glabrous. Leaf sheaths 3-5 $\mathrm{cm}$ long, linear, glabrous, persistent; ligules membranous with $0.4-0.6 \mathrm{~mm}$ long hairs at apex; leaf blades 5-18 × 0.2-0.25 cm, linear-lanceolate, convolute or sometimes equitant, surfaces ciliate adaxially and glabrous, ribbed abaxially, acuminate to attenuate at apex. Racemes 6-13 cm long, solitary, spike-like, 6-12 spikelets loosely arranged in rachis; rachis 4-6 mm long, stout, angular, scabridulous; peduncle 3-11 cm long, glabrous, ribbed. Spikelets 10-25 × 2-3 mm, linear, distant, dorsiventrally flattened, leaden green, 6-9flowered, acute at apex, disarticulation of florets from above glumes and in between florets; callus bearded, hairs 1.5-1.8 mm long; rachilla $0.5-1 \mathrm{~mm}$ long, glabrous, straight or zig zag, not persistent; lower glumes 4-4.5 × 0.7-1 mm, asymmetrical, ovate-lanceolate, 1-nerved, 1-keeled; keels slightly scabrid, acuminate or mucronulate at apex; upper glumes 4.5-5.5 × 1.5-2 mm, elliptic-lanceolate, 1nerved, 1-keeled, apex dentate, mucronate, mucro 0.3-0.4 mm long; lemmas 8-9.5 × 1.5-2 mm (including awn), elliptic-lanceolate, 2-lobed, 3nerved, slightly keeled, 1-awned, awns scabrid, straight, slightly bend when mature, 3-6 mm long, lateral lobes sharply acuminate or sometimes look like a minute awn, lobes 1-1.5 mm long; paleas 3$3.5(-4-5) \times 1.4-1.6 \mathrm{~mm}$, elliptic-oblanceolate, broadly winged, hyaline, 2-keeled, keels ciliate, emarginate or 2-lobed with a minute central notch at the apex; lodicules 2, c. $0.4 \mathrm{~mm}$ long, slightly cordate; stamens 3, anthers 1.5-2 mm long, oblong, filaments c. $0.3 \mathrm{~mm}$ long, slender, glabrous; ovary c. $0.5 \mathrm{~mm}$ long, obovate, styles $2,0.8-1 \mathrm{~mm}$ long, slender, hyaline, stigma $0.7-1 \mathrm{~mm}$ long, plumose, creamy white to purple violet. Caryopses $0.45-0.7$ $\times 0.2-0.3 \mathrm{~mm}$, narrowly oblong, brown.

Flowering \& fruiting: Flowering and fruiting from August to October.

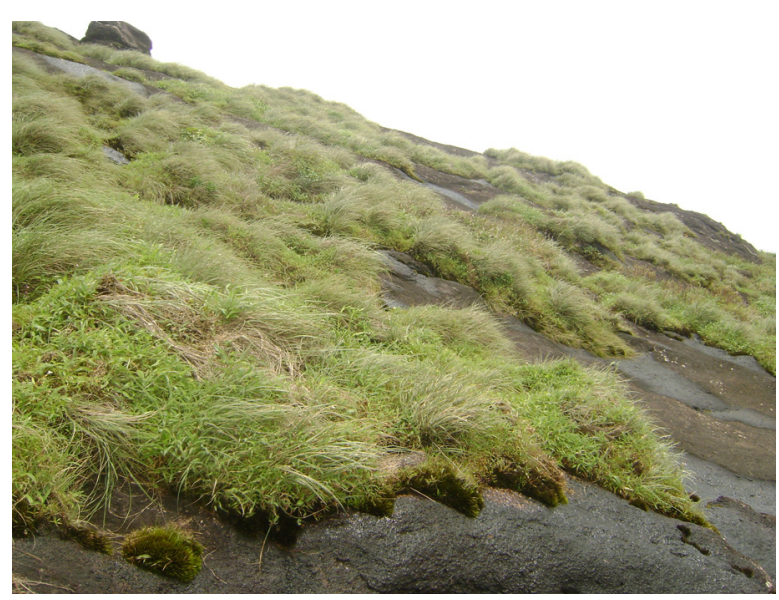

Fig. 44. Tripogon velliangiriensis Murug. \& V. Balas. at Velliangiri hills in Coimbatore district (Photo by A.K. Pradeep). 


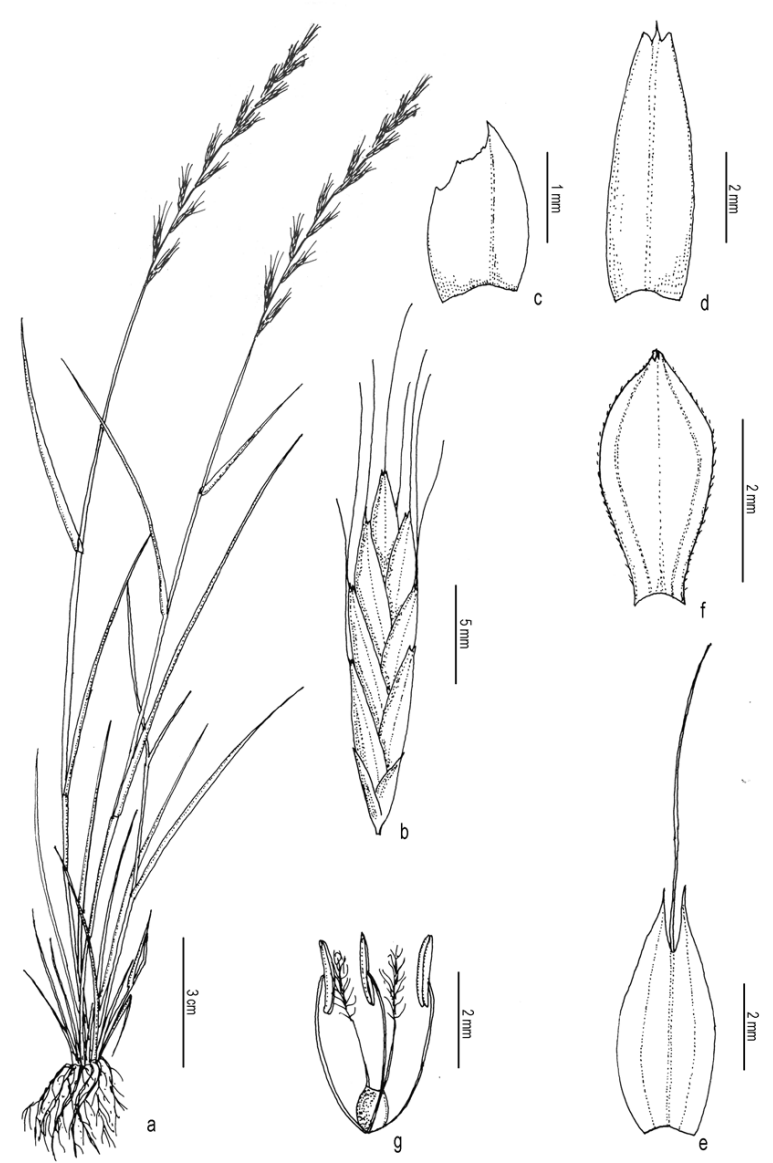

Fig. 45. Tripogon velliangiriensis Murug. \& V.Balas.: a. Habit; b. Spikelet; c. Lower glume; d. Upper glume; e. Lemma; f. Palea; g. Stamen \& pistil.

Habitat: This species grows between 1200-2200 m elevations on rocks and wet rocky hillsides in association with Cyanotis concanensis Hassk., C. cristata (both Commelinaceae), Cymbopogon martini (Roxb.) Will.Watson, Tripogon bromoides, T. filiformis, T. sivarajanii (all Poaceae), Henckelia humboldtiana, H. wightii (C.B.Clarke) A.Weber \& B.L.Burtt (Gesneriaceae) and Impatiens sp. (Balsaminaceae).

Distribution: Endemic to South India.

Specimens examined: INDIA, Kerala, Idukki district, Peerumedu, Parunthpara, 16.10.2015, K. Thoiba \& A.K. Pradeep 146621, 146628, 146629 (CALI); Palakkad district, Nelliyampathy, Keshavanpara, 07.11.2013, Thoiba K. 134492 (CALI); Thrissur district, Parambikulam Tiger Reserve, Karimala hills, 23.10.1990, Sasidharan s.n.; Ibid., 26.09.2013,
K. Thoiba 134454; (CALI). Tamil Nadu, Coimbatore district, Velliangiri hills, 30.09.2016, A.K. Pradeep \& Nikhil Krishna 146725, 146700 (CALI).

Notes: Tripogon velliangiriensis was first described from the Velliangiri hills in Coimbatore district of Tamil Nadu by Murugesan and Balasubramaniam (2008). They distinguished it from $T$. wightii by its "leaves being longer than the inflorescence, 15-20 cm long; lower glume not toothed, linear-lanceolate, 6-6.5 mm long, awned, awns 1.5-2.5 mm long; upper glume 2toothed, linear-lanceolate, 8-9.5 mm long, awned, awns 2-2.5 mm long; median awn of lemma 10.5-12 mm long; straight, lobes of lemma acuminate, awned, awn 1.5-2 mm long”. Subsequently, Newmaster et al. (2008) published $T$. copei from the same hills of Velliangiri in Coimbatore district of Tamil Nadu. The type (Ragupathy \& Newmaster 55277) of this species was reportedly deposited at OAC and $\mathrm{KASCH}$. The authors tried in vain to locate the isotype of this taxon at $\mathrm{KASCH}$ and no holotype was available at OAC (Ragupathy, personal communication dated 14.07.2016).

The authors collected a good number of specimens from different areas of Velliangiri and adjoining hill ranges. A critical examination of protologue and available materials from the type locality shows that the floral characters used to delimit T. copei from $T$.velliangiriensis are variable. The authors have observed populations of T. velliangiriensis with longer leaves, asymmetrical lower glumes, exceptionally symmetrical at the apical spikelets, almost straight median awns, and palea cleft at apex. Even though the type specimens were unavailable for our studies, judging from the description and illustrations of $T$. copei provided in the protologue and a number of specimens collected from the type locality, we prefer to reduce $T$. copei to synonymy of T. velliangiriensis.

Tripogon wightii Hook.f., Fl. Brit. India 7(22): 286. 1896; C.E.C.Fisch. in Gamble, Fl. Madras 3: 
1833. 1934; Bor, Grass. Burma Ceylon India \& Pakistan 524. 1960; S.Moulik, Grass. Bam. India 2: 623. 1997; Pull., Fl. Andhra Pradesh 3: 1269. 1997; Kabeer \& V.J.Nair, Fl. Tamil Nadu Grass: 182. 2009; Sasidh., Biodiv. Doc. Kerala-Fl. Pl.: 595. 2004. Lectotype (designated by Thoiba \& Pradeep, 2018): INDIA, Mysore, Bellary district, 1834. Wight 1793 (K [K000245012 digital image!]).

Figs. $46,47 \& 50$

Caespitose perennials. Culms $20-35 \mathrm{~cm}$ high; stoloniferous or not, nodes glabrous. Leaf sheaths $2.3-4 \mathrm{~cm}$ long, linear, ribbed, persistent, inrolled to the culm; ligules indistinct with a tuft of 4-6 $\mathrm{mm}$ long hairs at apex; leaf blades 5-15 × 0.2-0.3 $\mathrm{cm}$, equitant, convolute, margins smooth, minutely ciliate adaxially, glabrous abaxially, margins minutely serrulate towards apex, acuminate to attenuate at apex. Racemes 6-15 cm long, solitary, spike-like, slender, with 8-12 spikelets loosely arranged in rachis; rachis stout, flat to triquetrous and serrulate, 3-7 mm long; peduncles $7-11 \mathrm{~cm}$ long, glabrous. Spikelets $13-30 \times 0.5-0.7 \mathrm{~mm}$ (excluding awns), oblong, distant, dorsiventrally flattened, olive to dark green or yellowish, 5-16flowered, disarticulation of florets from above glumes and in between florets; callus bearded, hairs 1-1.2 mm long; rachilla 1-1.2 mm long, glabrous to scabrid, almost straight, not persistent; lower

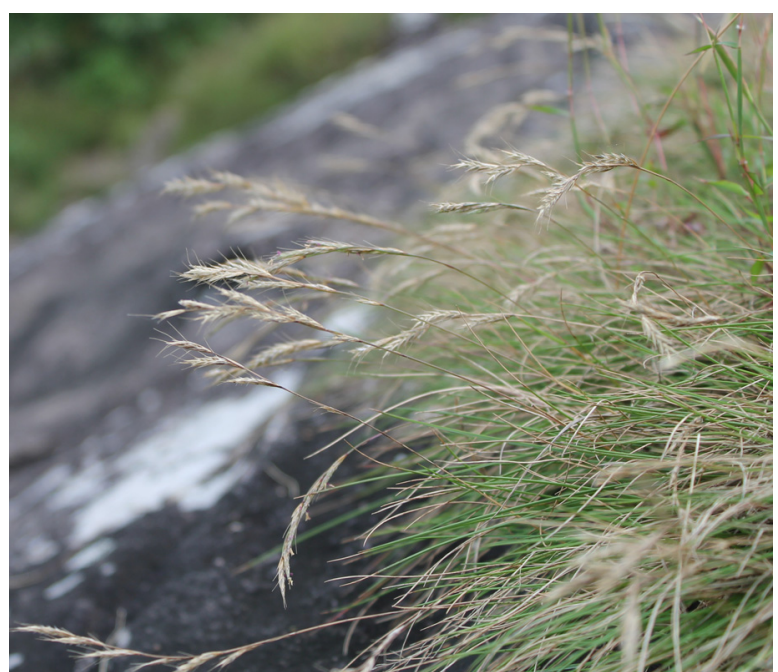

Fig. 46. Tripogon wightii Hook.f. in its natural habitat at Nelliyampathy hills in Palakkad district (Photo by K. Thoiba).

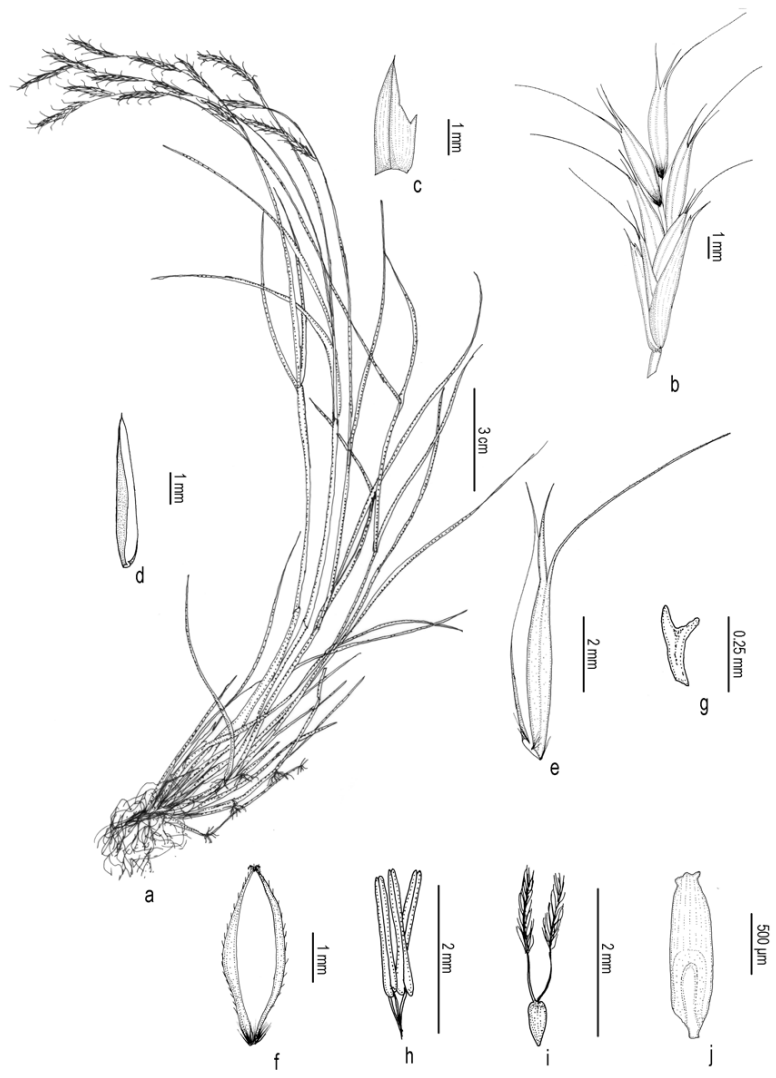

Fig. 47. Tripogon wightii Hook.f.: a. Habit; b. Spikelet; c. Lower glume; d. Upper glume; e. Lemma; f. Palea; g. Lodicule; h. Stamen; i. Pistil; j. Caryopsis.

glumes 3.6-4.8 × 0.5-0.7 mm, oblong-lanceolate, asymmetrical,1-nerved, 1-keeled, keels slightly scabrid, acuminate at apex, uppermost one with 1$1.3 \mathrm{~mm}$ long arista at apex; upper glumes 5-7 × 1$1.2 \mathrm{~mm}$, elliptic-lanceolate, 1-nerved, 1-keeled, apex dentate, 1-awned, awns 1-1.5 mm long, aristate at sinus; lemmas 4.8-6.2 $\times 1.4-2 \mathrm{~mm}$, ovatelanceolate, 2-lobed, lobes acute-acuminate,1.2-1.5 mm long, 3-nerved, slightly keeled, 1-awned, median awns 6-8 mm long, scabrid, straight to curved at maturity; paleas $2.3-4.2 \times 1-1.5 \mathrm{~mm}$, obovate to elliptic, broadly winged, surface pubescent, 2-keeled, ciliate along margins, sharply 2-lobed with a central notch at apex; lodicules 2 , 0.3-0.4 mm long, truncate; stamens 3, anthers 1-2 mm long, oblong, filaments $0.3-0.5 \mathrm{~mm}$ long, slender, glabrous; ovary $0.3-0.5 \times$ c. $0.25 \mathrm{~mm}$, obovate, styles 0.7-1.2 mm long, slender, hyaline, stigma $1.2-1.3 \mathrm{~mm}$ long, plumose, creamy white. Caryopses $1.4-2.2 \times$ $0.5 \mathrm{~mm}$, oblong-elliptic, light brown. 
Flowering \& fruiting: Flowering and fruiting from August to January.

Habitat: Occuring in seasonally wet hill slopes in association with Arundinella ciliata Nees, Tripogon bromoides, T. sivarajanii (all Poaceae), Cyanotis tuberosa, Murdannia semiteres (both Commelinaceae) and Henckelia incana (Gesneriaceae).

Distribution: Endemic to Peninsular India.

Specimens examined: INDIA, Andhra Pradesh, Chittoor district, Horsleykonda, 08.1989, J.S. Gamble 20920 (CAL). Kerala, Ernakulam district, Shoolamudi, Variyam, 14.12.2016, Nikhil Krishna \& K. Thoiba 146741 (CALI); Palakkad district, Nelliyampathy, 07.11.2013, K. Thoiba 134490, 146679 (CALI); Thrissur district, Sholayar, valve house, 11.12.2013, K. Thoiba 138031 (CALI). Tamil Nadu, Coimbatore district, Valparai, Shivamalai, 13.12.2013, K. Thoiba138011 (CALI); Nilgiri district, Pakasuramalai, 29.08.1957, K.M. Sebastine 3944 (CAL).

Notes: Tripogon wightii differs from other species in having leaves shorter than racemes, leaf blades equitant, spikelets being large sized (up to $20 \mathrm{~mm}$ long), awns in lemma straight when young and recurved at maturity, awns never more than twice as long as the lemma, and palea notched at apex.

Tripogon zeylanicus Nees ex Steud., Syn. Pl. Glumac. 1(3): 301. 1854; Thwaites, Enum. Pl. Zeyl. 374. 1864. Lectotype (designated here): CEYLON, s.d., C.P. Thwaites 281 (K [K000907445 digital image!]; isolecto CAL!).

Tripogon anantaswamianus Sreek., V.J.Nair \& N.C.Nair, Bull. Bot. Surv. India 25(1-4): 185, 1983. syn.nov; P.V.Sreek. \& V.J.Nair, Fl. Kerala Grass: 399, t. 82. 1991; S.Moulik, Grass. Bamb. India 2: 620, t. 137. 1997; Kabeer \& V.J.Nair, Fl. Tamil Nadu-Grass 179. 2009; Sasidh., Biodiv. Doc. Kerala-Fl. Pl. 595. 2004. Type: INDIA, Kerala, Idukki district, Eravikulam Sanctuary, 15.11.1980, P.V. Sreekumar 69432 (holo CAL [CAL0000002442!]; iso K [K000245042 digital image!], MH [MH00001659!]).

Figs. $48-50$
Caespitose perennials. Culms $30-50 \mathrm{~cm}$ high erect; nodes glabrous, slightly geniculate, 1-noded. Leaf sheaths 4-10 cm long, linear, glabrous or hairy; ligules a small fine membrane with a tuft of 1-2 $\mathrm{mm}$ long hairs at apex; leaf blades $25-40 \times 0.3-0.5$ $\mathrm{mm}$, linear-lanceolate, flat-convolute, more hairy towards collar, sparsely hairy along margins and both surfaces, acuminate to attenuate at apex. Racemes 10-30 cm long, solitary, slender, spikelets loosely or tightly arranged in rachis; rachis 3-7 $\mathrm{mm}$ long, stout, glabrous or scabrid; peduncles 10$15 \mathrm{~cm}$ long, glabrous. Spikelets 8-15.5 × 3-3.5 $\mathrm{mm}$, ovate-lanceolate, linear, dorsiventrally flattened, leaden green, 7-15-flowered; callus bearded, hairs $0.5-1 \mathrm{~mm}$ long; rachilla $1-1.3 \mathrm{~mm}$ long, glabrous, almost straight, not persistent; lower glumes 4-5 × 0.75-1 mm, asymmetrical, ovate-lanceolate, 1-nerved, 1-keeled, keels slightly scabrid, awned at apex, awns 1-1.5 mm long; upper glumes 7-9 × 0.5-1 mm, elliptic-lanceolate, 1-nerved, 1-keeled, apex 2-lobed, mucronate or awned, awns scabrid, 1-2 mm long; lemmas 7-8 $\times 1.5-2.5 \mathrm{~mm}$ (including awn), oblong-lanceolate, slightly keeled, 6-lobed, 3-nerved, 3-awned, lateral awns and median awns sub-equal or sometimes reduced, lateral lobes on either side of each lateral awn 0.25-0.5 mm long, awns scabrid, apex straight or geniculate, median awns $c .4 \mathrm{~mm}$ long, lateral awns $0.5-3 \mathrm{~mm}$ long, lateral lobes 1$1.5 \mathrm{~mm}$ long, acuminate, margins scabrid, apex sometimes bi-lobed; paleas 3-4 × 1-1.4 mm, elliptic-oblanceolate, hyaline, 2-keeled, ciliate, acuminate at apex; lodicules 2, 0.3-0.5 mm long, truncate; stamens 3, anthers 1.3-2 mm long, oblong, filaments $0.5-0.75 \mathrm{~mm}$ long, slender, glabrous; ovary 0.3-0.6 mm, obovate, styles 2, 1$1.2 \mathrm{~mm}$ long, slender, hyaline, stigma $0.7-1.2 \mathrm{~mm}$ long, plumose, creamy white. Caryopses 1.2-1.8 $\times$ 0.3-0.4 mm, narrowly oblong, cylindrical, brown.

Flowering \& fruiting: Flowering and fruiting from September to early December.

Habitat: This species is usually found growing in seasonally wet rock crevices and Ghat road sides at 
elevations between 700-2000 $\mathrm{m}$ in association with Arundinella pumila, Eragrostis collinensis, Tripogon bromoides, T. ravianus, T. sivarajanii (all Poaceae), Cyanotis tuberosa and C. villosa (Spreng.) Schult. \& Schult.f. (both Commelinaceae).

Distribution: Endemic to South India and Sri Lanka. Specimens examined: INDIA, Kerala, Idukki district, Eravikulam Sanctuary, 27.08.1980, P.V. Sreekumar 68403 (BSID, MH); Ibid., 15.12.2014, K. Thoiba \& A.K. Pradeep 137571a, 137571b; Ibid., 12.12.2015, K. Thoiba \& A.K. Pradeep 146697 (CALI); Ibid., 16.11.1980, P.V. Sreekumar 69429 (BSID). s.loc., s.d., C.N. Sunil 1775 (CALI); Poovanpara, 07.09.1997, S.D. Biju 36185 (TBGT); Alampetty, Marayoor-Chinnar road, 26.10.2017, A.K. Pradeep \& K. Thoiba 146753 (CALI); Munnar, 14.11.1980, P.V. Sreekumar 68460 (BSID); Wayanad district, Chembra, 12.10.2012, Remya

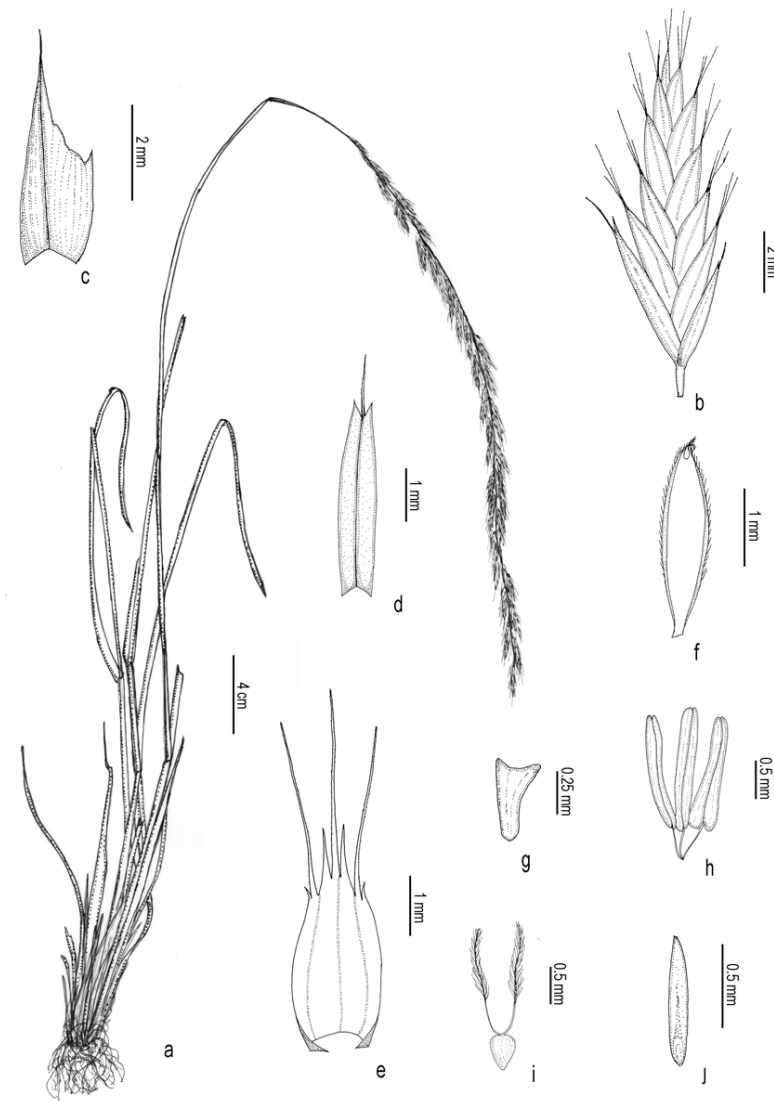

Fig. 48. Tripogon zeylanicus Nees ex Steud.: a. Habit; b. Spikelet; c. Lower glume; d. Upper glume; e. Lemma; f. Palea; g. Lodicule; h. Stamen; i. Pistil; j. Caryopsis.

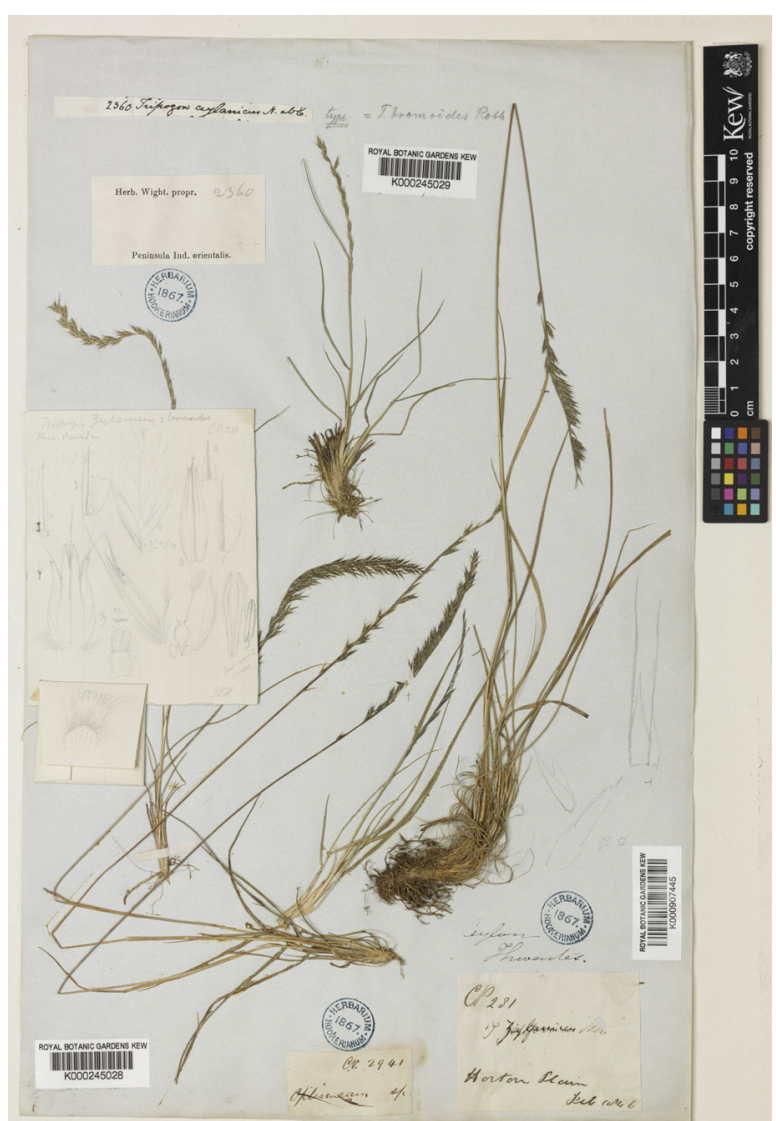

Fig. 49. Lectotype of Tripogon zeylanicus Nees ex Steud. (K000245028) designated by here. (C) The Board Trustees for the Royal Botanic Gardens, Kew. Reproduced with permission.

J. \& Prasanna 74507 (TBGT). Tamil Nadu, Coimbatore district, Valparai, 28.01.1978, M. Chandrabose 57709 (MH); Dindigul district, Kodaikanal, Shevaroy hills, 09.1905, C.A. Barber 7254; Observatory hills, 09.1905, C.A. Barber 7254a; Palani hills, Poombarai road, 04.07.1901, Bourne 2111 (MH); Kodaikanal, Palani hills, Berijam road, 06.12.1986, K.M. Matthew \& M. Charles 47756; Kukkal, Boothanachiammankoil hill, 19.10.1987, K.M. Matthew 50803; KodaiBerijam road, $16^{\text {th }} \mathrm{km}, 17.12 .1989$, S. Perianayagam 53973; Vandaravu, 18.12.1989, K.M. Matthew \& K.T. Matthew 54018; Berijam slopes, 09.08.1984, K.M. Matthew 40785 (RHT); Nilgiri district, Avalanche, 14.10.1972, K. Vivekananthan 42950; Bison swamp, 11.06.1970, B.V. Shetty 34196a; Mukurthi National Park, 16.05.1971, J.L. Ellis 38476 (MH); s.loc., 24.05.2016, C.N. Sunil 1775 (CALI); Ootacamund, 05.08.1900, C.A. Barber 


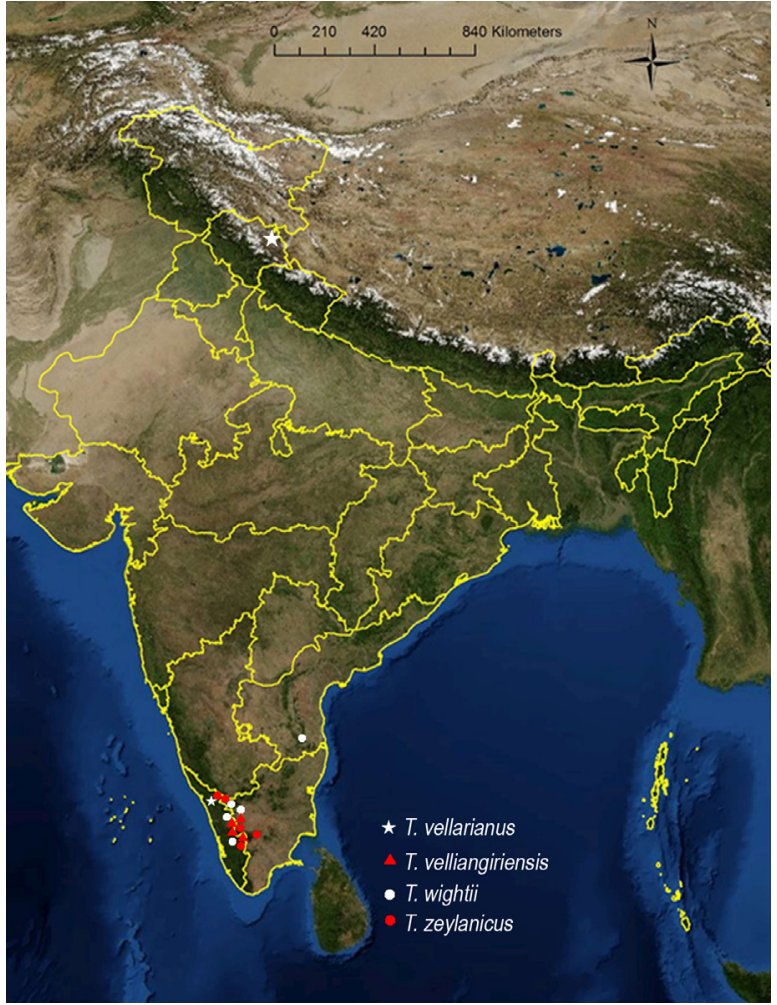

Fig. 50. Distribution of Tripogon vellarianus Pradeep, T. velliangiriensis Murug. \& V. Balas., T. wightii Hook.f. and T. zeylanicus Nees ex Steud. in India [drawn using ESRI (2011) ArcGIS Desktop: Release 10].

2652; Wenlock downs, Ootacamund, 11.11.1956, B.D. Patil 945 (CAL); s.loc., s.d., Wight 1871 (CAL); s.loc., s.d., s.coll. $56(\mathrm{MH})$.

Notes: Tripogon zeylanicus was originally described without giving a reference to a specimen. However, Steudel (1854) while validating the name provided a reference to the country of collection as "Ins Zeylon". A search for specimen used for the description of T. zeylanicus from Sri Lanka, resulted in two specimens bearing the same collection number (C.P. 281) one at CAL and the other at $\mathrm{K}$ which was also cited by Thwaites (1864). There are five specimens of various sizes mounted on the single sheet at $\mathrm{K}$ (K000907445 digital image!). An illustration with all its floral parts derived from C.P. 281 is seen attached on the middle left and side of the sheet. All parts in the drawing agreeing well with the description provided by Steudel and subsequently by Thwaites. Presumably it is the sheet used by Steudel for describing T. zeylanicus and is selected here as the lectotype. The left hand side specimen with the illustration affixed, probably with a young inflorescence cannot be ascertained to be T. zeylanicus. Tripogon anantaswamianus described by Sreekumar et al. (1983b) exactly correspond to $T$. zeylanicus and hence it is reduced to synonymy.

\section{Acknowledgements}

We are grateful to the state Department of Forests in India for granting permission to conduct field studies in the forest reserves and protected areas. We wish to thank the Directors and Curators of the following Herbaria AHMA, BLAT, BSI, BSID, CAL, CALI, DEV, FRC, Goa University Herbarium, JCB, KFRI, MH, RHT, SUK and TBGT for allowing us to consult the herbarium and library. The support provided by the various circles of Botanical Survey of India is gratefully acknowledged. We gratefully remember the support provided by late Dr. J.F. Veldkamp, National Herbarium Netherland, Leiden during this work. We are also thankful to Dr. B. Ravi Prasad Rao (SKU), Dr. C.N. Sunil (SNM College Herbarium, Kerala), Dr. Kumar Vinod Gosavi (HPT Arts and RYK Science College, Nashik), who provided relevant specimens for our studies.

\section{Literature Cited}

ARUMUGAM S. \& C. MURUGAN 2017. A new species of Tripogon (Poaceae: Chloridoideae: Tripogoninae) from India. Indian Journal of Forestry 40(2): 159-162.

BOR N.L. 1960. The grasses of Burma, Ceylon, India $\mathcal{E}$ Pakistan. Pergamon Press, London.

CHEN S.L. \& S.M. PHILLIPS 2006. Tripogon Roem. \& Schult. In: WU Z.Y., RAVEN P.H. \& D.Y. HONG. (eds.), Flora of China (Poaceae). Volume 22. Science Press, Beijing and Missouri Botanical Garden Press, St. Louis. pp. 466-469.

CHORGHE A., DEY S., PRASAD K., PRASANNA P.V. \& Y.V. RAO 2015. Tripogon mahendragiriensis sp. nov. (Poaceae) from the Eastern Ghats of Odisha (Orissa) state, India. Nordic Journal of Botany 33: 655-658. https:/ /doi.org/10.1111/njb.00874

CHORGHE A., RASINGAM L., PRASANNA P.V. \& M.S. 
RAO 2013. Tripogon tirumalae (Poaceae), a new species from the Seshachalam hills of Andhra Pradesh, India. Phytotaxa 131(1): 17-22. https://doi.org/10.11646/ phytotaxa.131.1.3

CLAYTON W.D. \& S.A. RENVOIZE 1986. Genera Graminum. Grass genera of the World. Kew. Royal Botanic Gardens, Kew.

CLIFFORD H.T. \& P.D. BOSTOCK 2007. Etymological dictionary of grasses. Springer-Verlag, Berlin.

DEY S. \& P.V. PRASANNA 2020[“2019”]. Taxonomic status of some recently described species of Tripogon Roem. \& Schult. (Poaceae) from India. Journal of Economic and Taxonomic Botany 43(1-4): 5-9.

ESRI 2011. ArcGIS Desktop: Release 10. Environmental System Research Institute, Redlands.

FABILLO M. 2015. Leaf and inflorescence structure and phylogenetics of Tripogon and affiliated genera (Poaceae: Chloridoideae). Ph.D. Thesis, Queensland University of Technology (QUT), Queensland. https:// eprints.qut.edu.au/83726

HONDA M. 1927. Tripogon longiaristatus. Botanical Magazine 41: 11-12.

HOOKER J.D. 1896. Flora of British India. Volume 7. L. Reeve \& Co., London. http://doi.org/10.5962/ bhl.title. 678

KABEER K.A.A., NAIR V.J. \& G.V.S. MURTHY 2009. Tripogon borii - a grass species new to science from India. Bulletin of the Botanical Survey of India 50: 115-118.

MURUGESAN M. \& V. BALASUBRAMANIAM 2008. Tripogon velliangiriensis (Poaceae) - a new species from Tamil Nadu, India. Indian Journal of Forestry 31(1): 109111.

MURUGESAN M., ARUMUGAM S. \& K.A.A. KABEER 2017. Tripogon paramjitianus (Poaceae: Chloridoideae) a new species from the Western Ghats of Tamil Nadu, India. Indian Journal of Forestry 40(3): 285-287.

NEWMASTER S.G., BALASUBRAMANIAM V., MURUGESAN M. \& S. RAGUPATHY 2008. Tripogon cope (Poaceae: Chloridoideae), a new species supported by morphometric analysis and a synopsis of Tripogon in India. Systematic Botany 33: 695-701. https://doi.org/ 10.1600/036364408786500253

NOLTIE H.J. 1999. Notes relating to the Flora of Bhutan: XXXIX. Gramineae II. Edinburgh Journal of Botany 56: 381-404. https://doi.org/10.1017/S0960428600001347

PETERSON P.M., ROMASCHENKO K. \& Y.H. ARRIETA 2016. A molecular phylogeny and classification of the Cynodonteae (Poaceae: Chloridoideae) with four new genera: Orthacanthus,
Triplasiella, Tripogonella, and Zaqiqah; three new subtribes: Dactyloteniinae, Orininae, and Zaqiqahinae; and a subgeneric classification of Distichlis. Taxon 65(6): 1263-1287. https://doi.org/10.12705/656.4

PHILLIPS S.M. \& E. LAUNERT 1971. A revision of the African species of Tripogon Roem. \& Schult. Kew Bulletin 25(2): 301-322. https://doi.org/10.2307/4103229

PHILLIPS S.M. \& S.L. CHEN 2002. The genus Tripogon (Poaceae) in China. Kew Bulletin 57(4): 911-924. https:/ /doi.org/10.2307/4115721

PRADEEP A.K. \& C.N. SUNIL 1999. Two new species of Tripogon (Poaceae) from India. Sida 18(3): 809-814. https://www.jstor.org/stable/41968899

RAO B.R.P. \& M. ANIL KUMAR 2018. Tripogon umaganeshii (Poaceae: Chloridoideae: Tripogoninae), a new species from India. Indian Journal of Forestry 41(1): 97-101.

RASINGAM L. \& J. SWAMY 2018. A new species of Tripogon (Poaceae: Chloridoideae: Tripogoninae) from Nallamala forests, Telangana, India. Phytotaxa 351(4): 296-300. https://doi.org/10.11646/phytotaxa.351.4.6

ROEMER J.J. \& J.A. SCHULTES 1817. Systema Vegetabilium: Secundum Classes, Ordines, Genera, Species. Cum Characteribus Differentiis et Synonymiis. Vol. 2. (Ed. 15). Stuttgartgardtiae: J.G. Cottae. https://doi.org/ 10.5962/bhl.title.825

RÚGOLO DE AGRASAR Z.E. \& A.S. VEGA 2004. Tripogon nicorae, a new species and synopsis of Tripogon (Poaceae: Chloridoideae) in America. Systematic Botany 29 (4): 874-882. https://doi.org/10.1600/ 0363644042451125

SREEKUMAR P.V., NAIR V.J. \& N.C. NAIR 1983a. Tripogon narayanii--a new species of Poaceae from Kerala, India. Journal of Bombay Natural History Society 80(1): 196-198.

SREEKUMAR P.V., NAIR V.J. \& N.C. NAIR 1983 b. Tripogon anantaswamianus Sreek., V.J. Nair et N.C. Nair - a new grass from Kerala, India. Bulletin of the Botanical Survey of India 25(14): 185-187.

STAFLEU F.A. \& R.S. COWAN 1979. Taxonomic Literature: A selective guide to botanical publications and collections with dates, commentaries and types, second edition, Volume 2: H-Le. Regnum Vegetabile 98. Bohn, Scheltema \& Holkema, Utrecht, The Hague. https:// doi.org/10.5962/bhl.title.48631

STEUDEL E.G. 1854. Synopsis Plantarum Glumacearum, Vol. 1 - Gramineae, J.B. Metzler, Stuttgartiae. https:// doi.org/10.5962/bhl.title.471

SUNIL C.N. \& A.K. PRADEEP 2001. Another new species of Tripogon (Poaceae) from India. Sida 19(4): 803-806. https://www.jstor.org/stable/41967930 
SUNIL C.N., PRADEEP A.K. \& K. THOIBA 2015. Tripogon idukkianus (Poaceae: Chloridoideae), a new species from India. Phytotaxa 202(4): 294-297. https:// doi.org/10.11646/phytotaxa.202.4.9

TEERAWATANANON A. \& S. SUNGKAEW 2012.

Tripogon purpurascens (Chloridoideae: Poaceae): a native Thai grass recently recognized. Thai Forest Bulletin (Botany) 40: 130-133.

THIERS B. (continuously updated). Index Herbariorum: A global directory of public Herbaria and associated staff. New York Botanical Garden's Virtual Herbarium. Available at: http://sweetgum.nybg.org/science/ih (Accessed on 17.11.2019).

THOIBA K. \& A.K. PRADEEP 2014. Tripogon malabarica (Poaceae: Chloridoideae: Tripogoninae), A new species from India, Kerala. Journal of Botanical Research Institute of Texas 8(2): 523-527. https://www.jstor.org/stable/ 26549400

THOIBA K. \& A.K. PRADEEP 2018. Lectotypification of three names in the genus Tripogon (Poaceae). Phytotaxa
350(2): 177-181. https://doi.org/10.11646/ phytotaxa.350.2.8

THOIBA K., MANUDEV K.M. \& A.K. PRADEEP 2016. Two new species of Tripogon (Poaceae: Chloridoideae: Tripogoninae): from India. Phytotaxa 272(2): 125-133. https://doi.org/10.11646/phytotaxa.272.2.3

THOIBA K., PRADEEP A.K. \& C.N. SUNIL 2015. Tripogon bimucronatus (Poaceae: Chloridoideae: Tripogoninae), a new species from India. Gardens' Bulletin, Singapore 67(1): 151-157. https://doi.org/ 10.3850/S2382581215000162

THWAITES G.H.K. 1864. Enumeratio Plantarum Zeylaniae: An Enumeration of Ceylon Plants. Dulau \& Co., London. https://doi.org/10.5962/bhl.title.574

WATSON L., MACFARLANE T.D. \& M.J. DALLWITZ 1992. Tripogon Roem. \& Schult. The Grass Genera of the World version 11. Available at: https://www.deltaintkey.com/grass/www/tripogon.htm (Accessed on 15.11.2019). 